\title{
A Review of the Phytochemistry, Traditional Uses, and Biological Activities of the Genus Ballota and Otostegia'
}

Authors

Sergio Rosselli ${ }^{1}$, Gianfranco Fontana ${ }^{2}$, Maurizio Bruno ${ }^{2}$

Affiliations

1 Department of Agricultural, Food and Forest Sciences (SAAF), University of Palermo, Viale delle Scienze, Parco d'Orleans II, IT-90128 Palermo, Italy

2 Department of Biological, Chemical and Pharmaceutical Sciences and Technologies (STEBICEF), University of Palermo, Viale delle Scienze, Parco d’Orleans II, IT-90128 Palermo, Italy

Key words

Ballota, Otostegia, secondary metabolites, antioxidant, antibacterial, antifungal

received January 24, 2019

revised June 5, 2019

accepted June 7, 2019

Bibliography

DOI https://doi.org/10.1055/a-0953-6165

Published online June 19, 2019 | Planta Med 2019; 85: 869-

910 @ Georg Thieme Verlag KG Stuttgart · New York |

ISSN 0032-0943

\author{
Correspondence \\ Prof. Sergio Rosselli \\ Department of Agricultural, Food and Forest Sciences (SAAF), \\ University of Palermo \\ Viale delle Scienze, Parco d'Orleans II - IT-90128 Palermo, \\ Italy \\ Phone: + 3909123897547 \\ sergio.rosselli@unipa.it
}

\section{ABSTRACT}

The 2 genera Ballota and Otostegia, belonging to the Lamiaceae family, are closely related taxonomically and found mainly in the Mediterranean area, Middle East, and North Africa. Since ancient times, they have been largely employed in traditional medicine for their biological properties such as antimicrobial, anti-inflammatory, antispasmodic, insecticidal, anti-malaria, etc. Phytochemical investigations of Ballota and Otostegia species have revealed that diterpenoids are the main constituents of the genera. A large number of flavonoids and other metabolites were also identified. This review, covering literature from 1911 up to 2018, includes traditional uses, chemical profiles (both of volatile and nonvolatile metabolites), and biological properties of all the taxa of these 2 genera studied to date.

\section{Introduction}

The genus Ballota, belonging to Lamiaceae family (Stachyoideae) Lamioideae subfamily) [1,2], is, apart from the South African endemic species Ballota africana (L.) Benth., naturally distributed in the Mediterranean, the Middle East and in North Africa. Some species (e.g., Ballota nigra L.s. I.) are also present over large areas of western, central, and northern Europe, and 4 species, whose status will be discussed later, in Somalia.

Ballota species are perennial herbs or small shrubs with branched and/or simple hairs, toothed and petiolate leaves, the inflorescence thyrsoid or racemoid sometimes has long and spinose bracteoles (sect. Acanthoprasium), and the calyx is mostly campanulate, purple to white.

A former classification of the genus identified the occurrence of 31 species ( 1 = Ballota integrifolia Benth. $-2=$ Ballota wettsteinii Rech. pat. $-3=$ Ballota frutescens (L.) Woods $-4=$ Ballota fruticosa Baker - 5 = Ballota somala Patzak - 6. Ballota andreuzziana Pamp. -
$7=$ Ballota acetabulosa (L.) Benth. $-8=$ Ballota undulata (Sieb, ex Fres.) Benth. $-9=$ Ballota pseudodictamnus (L.) Benth. $-10=$ Ballota damascena Boiss. - 11 = Ballota hildebrandtii Vatke et Kurtz 12 = Ballota hirsuta Benth. -13 = Ballota bullata Pomel $-14=$ B. africana (L.) Benth. $-15=$ Ballota aucheri Boiss. $-16=$ Ballota macrodonta Boiss. et Bal. - $17=$ Ballota larendana Boiss. et Heldr. - $18=$ Ballota rotundifolia C. Koch $-19=$ Ballota rupestris (Biv.) Vis. - 20 = Ballota macedonica Vand. - 21 = Ballota kaiseri V. Täckh. 22 = Ballota antilibanotica Post $-23=$ Ballota cristata Davis $-24=$ Ballota semanica Rech. f. $-25=$ Ballota labillardieri Briq. $-26=$ Ballota saxatilis Sieb. ex J. et C. Presl $-27=$ Ballota stachydiformis Höchst. $-28=$ Ballota philistea Bomm. $-29=$ Ballota platyloma

Dedicated to Professor Dr. Cosimo Pizza in recognition of his important contributions to natural product research on the occasion of his 70th birthday in 2019. 


\begin{tabular}{|c|c|}
\hline \multicolumn{2}{|c|}{ ABBREVIATIONS } \\
\hline ABTS & $\begin{array}{l}\text { 2,2'-azino-bis(3-ethylbenzothiazoline-6- } \\
\text { sulphonic acid) }\end{array}$ \\
\hline Ac & acetone \\
\hline AD & agar diffusion \\
\hline ALP & alkaline phosphatase \\
\hline AP & aerial parts \\
\hline $\mathrm{BuOH}$ & butanol \\
\hline CAT & catalase \\
\hline CQ & chloroquine \\
\hline CUPRAC & cupric ion reducing antioxidant capacity \\
\hline DPPH & 2,2-diphenyl-1-picrylhydrazyl \\
\hline EO & essential oil \\
\hline EtOAc & ethyl acetate \\
\hline EtOH & ethanol \\
\hline $\mathrm{F}$ & flowers \\
\hline FRAP & ferric reducing antioxidant power \\
\hline GSH & gluthatione \\
\hline L & leaves \\
\hline LPO & linoleic acid peroxidation \\
\hline MBC & minimum bactericidal concentration \\
\hline $\mathrm{MeOH}$ & methanol \\
\hline MIC & minimum inhibiting concentration \\
\hline MPO & myeloperoxidase \\
\hline MTT & $\begin{array}{l}\text { 3-(4,5-dimethylthiazol-2-yl)-2,5-diphenyltetra- } \\
\text { zolium bromide }\end{array}$ \\
\hline$n$-Hex & $n$-hexane \\
\hline NO & nitric oxide \\
\hline NOE & nuclear Overhauser effect \\
\hline ORAC & oxygen radical absorbance capacity \\
\hline PE & petroleum ether \\
\hline $\mathbf{R}$ & root \\
\hline S & stems \\
\hline SGOT & serum glutamic oxaloacetic transaminase \\
\hline SGPT & serum glutamine-pyruvate transaminase \\
\hline SOD & superoxide dismutase \\
\hline STZ & streptozotocin \\
\hline TB & total bilirubin \\
\hline TBARS & thiobarbituric acid reactive substances \\
\hline TEAC & Trolox equivalent absorbance capacity \\
\hline TG & triglycerides \\
\hline VLDL & very low density lypoproteins \\
\hline W & water \\
\hline WP & whole plant \\
\hline $\mathrm{x} / \mathrm{XO}$ & hypoxanthine/xanthine redox couple \\
\hline
\end{tabular}

hildebrandtii (Vatke \& Kurtz) Sebald, and Leucas stachydiformis (Benth.) Hochst. ex Briq., respectively [6]; B. integrifolia and $B$. wettsteinii are both considered synonyms of Acanthoprasium integrifolium (Benth.) Ryding (accepted name) [7]; B. frutescens, B. labillardieri, B. semanica, and B. rupestris are synonyms of Acanthoprasium frutescens (L.) Spenn. [7], B. saxatilis, B. saxatilis subsp. brachyodonta (Boiss.) P.H. Davis \& Doroszenko, and Ballota hispanica (L.) Benth., respectively [6].

The Plant List [6], which has been used to validate the scientific names of the species, includes more than 160 scientific plant names of species rank for the genus Ballota. Of these, only 30 are accepted species names.

The genus Otostegia (Lamiaceae family, Stachyoideae/Lamioideae subfamily), closely related to genus Ballota morphologically, with about 15 species, occurs in rather dry, often montane areas and semideserts [8]. There are 2 clearly disjoint centers of diversity for Otostegia: Central Asia to Afghanistan and northeastern Africa [9], although the genus is distributed from Cameroon to Saudi Arabia, Yemen, Egypt, Iran, and Central Asia to India [8].

In 2007, Scheen \& Albert [10] proposed to restrict the Otostegia genus, including only the following 11 species in it: Otostegia ellenbeckii Gürke, Otostegia ericoidea Ryding, Otostegia erlangeri Gürke, Otostegia fedtschenkoana Kudr., Otostegia fruticosa (Forssk.) Schweinf. ex Penzig, O. hildebrandtii, Otostegia migirtiana Sebald, O. modesta, Otostegia olgae (Regel) Korsh., Otostegia sogdiana Kudr., and Otostegia tomentosa A. Rich. The former members of Otostegia, O. somala and Otostegia aucheri, were transferred to Isoleucas and Moluccella, respectively. A new genus was erected for the 4 yellow-flowered species of Otostegia (Otostegia integrifolia Benth., Otostegia limbata [Benth.] Boiss, Otostegia michauxii Briq., Otostegia persica (Burm. f.) Boiss), for which the name Rydingia A.-C. Scheen \& V. A. Albert was proposed. Consequently, the 4 names actually accepted for these species are Rydingia integrifolia (Benth.) Scheen \& V.A. Albert, Rydingia limbata (Benth.) Scheen \& V.A. Albert, Rydingia michauxii (Briq.) Scheen \& V.A. Albert, Rydingia persica (Burm. f.) Scheen \& V. A. Albert [6].

The Plant List [6] is generally in agreement with this classification of genus Otostegia. It reports 46 name records of species rank for the genus Otostegia, of which only 10 species and 3 subspecies are accepted. In addition to the above-mentioned species [10], the Plant List added Otostegia nikitinae Scharasch. and Otostegia schennikovii Scharasch., while moving Otostegia bucharica B. Fedtsch., O. fedtschenkoana, O. olgae, and O. sogdiana to genus Moluccella [7].

In this review, a complete survey of the traditional uses, chemical constituents (both volatile and nonvolatile), and biological properties of species from the genera Ballota and Otostegia is provided.

The available information on these genera was collected from scientific databases and cover from 1911 up to 2018. The following electronic databases were used: PubMed, SciFinder, Science Direct, Scopus, Web of Science, and Google Scholar.

The search terms used for this review included Ballota, Otostegia, all the botanical names of the species, both accepted names or synonyms, phytochemical composition, EOs, traditional uses, activity, pharmacology, and toxicity. No limitations were set for languages. - Table 1 reports the taxa of Ballota and Otostegia investigated so far, their synonyms, and the accepted botanical names. 
- Table 1 Ballota s. I. and Otostegia s.I. taxa studied so far and their synonymous (accepted botanical name in bold).

\begin{tabular}{|c|c|}
\hline Taxa & Synonyms \\
\hline \multicolumn{2}{|l|}{ Ballota acetabulosa (L.) Benth. } \\
\hline \multicolumn{2}{|l|}{ Ballota africana (L.) Benth. } \\
\hline \multicolumn{2}{|l|}{ Ballota andreuzziana Pamp. } \\
\hline \multicolumn{2}{|l|}{ Ballota antalyensis Tezcan \& H. Duman } \\
\hline Ballota arabica Hochst. \& Steud. & Leucas urticifolia (Vahl) Sm. \\
\hline Ballota aucheri Boiss. & Otostegia aucheri Boiss. \\
\hline Ballota cinerea D. Don & Roylea cinerea (D. Don) Baill.; Roylea calycina (Roxb.) Briq.; R. elegans Wall. ex Benth. \\
\hline \multicolumn{2}{|l|}{ Ballota cristata P. H.Davis } \\
\hline Ballota deserti (Noë) Jury, Rejdali \& A. J. K.Griffiths & Marrubium deserti (Noë) Coss. \\
\hline \multicolumn{2}{|l|}{ Ballota glandulosissima Hub.-Mor. \& Patzak } \\
\hline \multicolumn{2}{|l|}{ Ballota hirsuta Benth. } \\
\hline Ballota hispanica (L.) Benth. & Ballota rupestris (Biv.) Vis. \\
\hline \multicolumn{2}{|l|}{ Ballota inaequidens Hub.-Mor. \& Patzak } \\
\hline Ballota lanata $\mathrm{L}$. & Panzerina Ianata (L.) Soják; Panzeria alaschanica Kuprian.; P. lanata (L.) Bunge \\
\hline \multicolumn{2}{|l|}{ Ballota larendana Boiss. \& Heldr. } \\
\hline \multicolumn{2}{|l|}{ Ballota latibracteolata P. H. Davis \& Doroszenko } \\
\hline \multicolumn{2}{|l|}{ Ballota macrodonta Boiss. \& Balansa } \\
\hline \multicolumn{2}{|l|}{ Ballota nigra $\mathrm{L}}$. \\
\hline \multicolumn{2}{|l|}{ B. nigra L. subsp. anatolica P. H.Davis } \\
\hline \multicolumn{2}{|l|}{ Ballota nigra subsp. foetida (Vis.) Hayek } \\
\hline Ballota nigra f. uncinata Beg, & Ballota nigra subsp. ruderalis (Sw.) Briq. \\
\hline \multicolumn{2}{|l|}{ Ballota philistaea Bornm. } \\
\hline Ballota pilosa Lour. & Leucas chinensis (Retz.) Sm.; L. mollissima subsp. chinensis (Benth.) Murata \\
\hline \multicolumn{2}{|l|}{ Ballota pseudodictamnus (L.) Benth. } \\
\hline \multicolumn{2}{|l|}{ Ballota pseudodictamnus subsp. lycia Hub.-Mor } \\
\hline \multicolumn{2}{|l|}{ Ballota rotundifolia K. Koch } \\
\hline Ballota rupestris (Biv.) Vis. & Ballota hispanica (L.) Benth. \\
\hline \multicolumn{2}{|l|}{ Ballota saxatilis Sieber ex C.Presl } \\
\hline \multicolumn{2}{|l|}{$\begin{array}{l}\text { Ballota saxatilis subsp. brachyodonta (Boiss.) P. H.Davis \& } \\
\text { Doroszenko }\end{array}$} \\
\hline Ballota schimperi Benth. & Otostegia fruticosa subsp. schimperi (Benth.) Sebald \\
\hline \multicolumn{2}{|l|}{ Ballota sechmenii Gemici \& Leblebici } \\
\hline \multicolumn{2}{|l|}{ Ballota undulata (Sieber ex Fresen.) Benth. } \\
\hline \multicolumn{2}{|l|}{ Otostegia fruticosa (Forssk.) Schweinf. ex Penzig } \\
\hline Otostegia fruticosa subsp. schimperi (Benth.) Sebald & Otostegia fruticosa subsp. schimperi (Benth.) Sebald \\
\hline Otostegia integrifolia Benth. & Rydingia integrifolia (Benth.) Scheen \& V. A.Albert \\
\hline Otostegia limbata (Benth.) Boiss. & Rydingia limbata (Benth.) Scheen \& V. A.Albert; Ballota limbata Benth. \\
\hline Otostegia persica (Burm.f.) Boiss. & Rydingia persica (Burm.f.) Scheen \& V. A.Albert; Ballota persica (Brum.f.) Benth \\
\hline Otostegia tomentosa A.Rich. & \\
\hline
\end{tabular}

\section{Traditional Uses}

Several plant species belonging to Ballota and Otostegia genera have been used in traditional medicine of many countries. A summary of their traditional use is presented in $>$ Table 2.

In Europe, the most utilized is, by far, $B$. nigra, a perennial herb native to the Mediterranean region and to central Asia, which can be found throughout Europe. It is also naturalized in Argentina,
New Zealand, and the eastern United States. Leaves of B. nigra were used as an antidote for rabid dog bites. It was used in the Balkanic area as a sedative/tranquilizer in cases of hysteria and hypochondria [31,32,37,38]. It is also used in Italy externally, for wound-healing properties $[33,36]$. Internally, in the Balkans, it is used as a sedative, a spasmolytic for stomach cramps and aches, for whooping cough, and to increase bile flow. It is also used to treat nervousness, upset stomach, nausea, and vomiting [30, 
38]. In Moldova, in the form of enemas and suppositories, it is used against worm infestation [31]. In northern Spain, it is used as insecticide and repellent against fleas [39]. In several parts of Turkey, its subspecies $B$. nigra L. subsp. anatolica, where it is known by different vernacular names, has been reported for the treatment of cold and flu [43], flatulence, and upset stomach [41] and as antiseptic for wounds, burns, and inflamed skin [40, 42]. B. acetabulosa, known as the Greek horehound, is a compact, evergreen subshrub, growing to $0.5 \mathrm{~m}$, native to southeast Greece, Crete, and western Turkey. In Turkey, the infusion of leaves is used for treatment of stomach ailments, where the leave poultice relieves abdominal pain and hemorrhoids $[11,12]$.

In the southern part of Africa, the only species present is B. africana, known as Cape horehound or "kattekruie." It is most common in the more arid, winter rainfall areas of the Cape. Its natural distribution stretches from the southern part of Namibia down to the West Coast and Cape Peninsula. Along this wide distribution, $B$. africana is usually found along streams and in the shelter of rocks and bushes. Externally, a leaf compress is applied on sick children's feet, on painful legs, inflamed joints, backache, head for headache, on cheek for toothache, on breasts for mastitis, wash for chilblained hands and feet, wounds, ointment on sores, and as poultice on boils. Orally, leaf infusion is used for stomach ache, influenza, fever, asthma, lung, and urinary infections, to treat convulsions in infants, to wean infants, and as cough syrup [13-15].

Ballota deserti (syn. Marrubium deserti) is a common endemic species in the northern and central Sahara. In Tunisia, it is employed in traditional medicine in the form of a decoction as a remedy for asthma, diabetes, and as a diuretic [25]. The internal usage of this species has been documented in the central Sahara using the infusion of its leaves for respiratory diseases, fever, colic, colds, cough, digestive troubles, helminthiasis, and nausea [23,24]. Another plant utilized in North Africa (High Atlas, Morocco, and West Algeria) is $B$. hirsuta, native to the western Mediterranean region, mostly abundant in Spain, Portugal, and North Africa. It is very popular with traditional healers (known as "uarimsa," "tougan'if-zi," or "tifziguiyin") as a cure for many diseases. The poultice of leaves and roots is used very often to treat subcutaneous lesions (contusion), rheumatic pains, and heal various wounds. The decoction of flowers is used externally as an antiseptic or orally against dental caries, whereas the flower infusion is utilized internally to treat gastrointestinal, gynecological and pediatric diseases [26,27].

In East Africa (Eritrea, Ethiopia), there are many reports of some Otostegia species concerning their usage in traditional medicine. The most numerous data are available for O. integrifolia (syn. $R$. integrifolia). O. integrifolia, commonly known as Abyssinian rose,

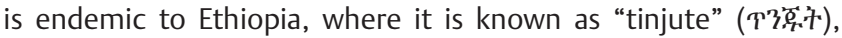
growing in the dry evergreen woodlands regions at altitudes of 1300-2800 m above sea level. It also grows in Eritrea and Yemen. In northern Ethiopia, it is commonly used to smoke utensils for sterilization. It is also a ritual custom for a mother to cleanse herself with the smoke on the tenth day after giving birth to a child before leaving her confinement to resume normal daily activities [56]. It has been largely employed as an insect repellent against fleas and mosquitos and as antimalarial [47, 50, 52, 53]. Inhalation of the smoke of burnt stems and leaves is used against evil eye $[48,51]$ and its juice, diluted with water, is drunk for treatment of stomach ache, vomiting, nausea, diarrhea, and dysentery [46, 51]. In the same geographical area, the juice of 0 . tomentosa and 0 . fruticosa has been similarly used against diarrhea [46], and the latter also against ascariasis [46] and tonsillitis [48].

O. fruticosa also grows in the Arabic peninsula where the infusion of flowering branches is used as a remedy for sun-stroke [49] or as an anti-paralytic and for eye diseases $[44,45]$.

In Pakistan, India and Iran, species belonging to Ballota and Otostegia genera have been largely employed in traditional medicine.

B. arabica (syn. Leucas urticifolia (Vahl) Sm.) is an annual herb distributed in the Punjab, Baluchistan, Sindh, and Rajputana desert of Pakistan. In Baluchistan, where it is known as "kubo" or "goma," the plant is used as a cure for fever. Furthermore, the decoction of the leaves and apical shoots is used as an abortifacient up to 3 mo of pregnancy. Infusions of the flowers are used to treat skin diseases. The plant is also used for the treatment of diarrhea, dysentery, uterine hemorrhages, dropsy, gravel, cystitis, calculus, bronchial catarrh, skin diseases, fever, and various types of mental disorders [16]. The decoction of leaves, roots and flowers of B. aucheri is topically employed in both Pakistan and Iran as hair tonic, for strengthening gums, dental cleaning and brightness, and prevention of hair loss $[17,18]$. While in Baluchistan, Iran, the decoction of leaves and flowers of 0 . persica ("golder") is drunk for treatment of diabetes, rheumatism, cardiac distress, palpitation, hypertension, cold, hyper lipidemia, gastric discomfort, headache, and as parasite repellent, sedative, laxative, carminative, and antipyretic [17]. In Pakistan, the largest number of ethno-pharmacological reports involve 0 . limbata. In fact, it is extensively utilized by traditional practitioners against several ailments since it possesses antispasmodic, antiulcer, antidepressant, sedative, and anxiolytic properties [68]. O. limbata is consumed for the treatment of children's gum problems and for remedial purposes in cases of ophthalmia [57-59,61,63]. Local, fresh leaves of $O$. limbata are crushed and then grounded and mixed with water to make the extract which is also used to cure eye infections. Due to its antiseptic and antibacterial properties [65], powder of dried leaves is mixed with butter and layered on wounds and boils in both humans and animals [59,65,69]. Dried plant powder is also utilized against jaundice $[61,63]$.

Ballota cinerea D. Don is vernacularly known as Karui, Titpatti, or Patkarru. WP parts are widely used as folk medicine in India and Nepal. Shoots are crushed and eaten with salt to strengthen the liver by local villagers. Young shoots are used as insect repellent for cattle during rainy season. Leaves and shoot extraction are used in scabs and other skin infections. AP are widely used to treat malaria and various liver disorders like jaundice, liver debility, and fever [20,21].

The traditional uses of Ballota and Otostegia species are wide and sometimes may be directly correlated to the content of some active class of compounds. Along with diterpenes that characterize these species, flavonoids and phenolic compounds, the latter ones often occurring as esters moieties, are the main constituents of the plant extracts and their antibacterial and anti-inflammatory activities are well documented in literature. They could be responsible for most of the claimed remedies. In the following sections the metabolic profiles and the biological activities of these plants have been analyzed. 
- Table 2 Ethnopharmacological uses of Ballota and Otostegia taxa.

\begin{tabular}{|c|c|c|c|c|}
\hline Species & $\begin{array}{l}\text { Vernacular } \\
\text { names }\end{array}$ & Area & Use & Ref \\
\hline \multirow[t]{2}{*}{ B. acetabulosa } & boz ot & Aydin, Turkey & stomach ailments, abdominal pain & [11] \\
\hline & & Balikesir, Turkey & hemorrhoid treatment & [12] \\
\hline \multirow[t]{2}{*}{ B. africana } & kattekruid, oulap & $\begin{array}{l}\text { Namaqualand, } \\
\text { South Africa }\end{array}$ & $\begin{array}{l}\text { stomach ache, headache, backache, wounds, pediatric, coughs and bronchitis, } \\
\text { chest ailments, toothache, burning feet, earache, convulsions, weaning, chil- } \\
\text { blained hands and feet, mastitis }\end{array}$ & [13] \\
\hline & kattekruid & South Africa & fever, cough, asthma, lung infections, influenza, insomnia, stress & {$[14,15]$} \\
\hline B. arabica & kubo, goma & Baluchistan, Pakistan & abortifacient, astringent, stimulant, hemostatic, anthelmintic, diuretic & [16] \\
\hline \multirow[t]{2}{*}{ B. aucheri } & golder & Baluchistan, Iran & $\begin{array}{l}\text { hair tonic, strengthening gums, dental cleaning and brightness, } \\
\text { prevention of hair loss }\end{array}$ & [17] \\
\hline & chashing & Gilgit Baltistan, Pakistan & hair tonic, dental cleaning & [18] \\
\hline \multirow[t]{3}{*}{ B. cinerea } & kori & Himachal Pradesh, India & stomachache, analgesic & [19] \\
\hline & karui & Uttarakhand, India & fever, jaundice, skin disease, malaria and most prominently in diabetes & {$[20,21]$} \\
\hline & $\begin{array}{l}\text { karui, titpati, } \\
\text { patkarru }\end{array}$ & Nepal, Kashmir & fever, jaundice, scabs, skin disease, malaria, insect repellent & {$[22]$} \\
\hline \multirow[t]{2}{*}{ B. deserti } & telheret, meriout & Central Sahara & $\begin{array}{l}\text { respiratory diseases, fever, colics, colds, cough, digestive troubles, } \\
\text { helminthiasis, nausea }\end{array}$ & {$[23,24]$} \\
\hline & & Tunisia & asthma, diabetes, diuretic & [25] \\
\hline \multirow[t]{2}{*}{ B. hirsuta } & $\begin{array}{l}\text { uarimsa, touga- } \\
\text { n'if-zi, tifziguiyin }\end{array}$ & High Atlas, Morocco & general health, gastrointestinal, gynecological, pediatric & [26] \\
\hline & & West Algeria & contusion, injuries and rheumatic pain & [27] \\
\hline \multirow[t]{2}{*}{ B. lanata } & & Mongolia & $\begin{array}{l}\text { treatment of pelvic inflammation and chronic pelvic inflammation, edema, } \\
\text { irregular menstruation, dysmenorrheal, amenorrhea, nephritis }\end{array}$ & {$[28]$} \\
\hline & gang ga' chung & Tibet & stomach, intestinal, and gynecological diseases & [29] \\
\hline \multirow[t]{9}{*}{ B. nigra } & & Sharr Mt., Macedonia & digestive & [30] \\
\hline & & Moldova & sedative, antispasmodic stimulant, vermifuge & [31] \\
\hline & crna kopriva & $\begin{array}{l}\text { Northeast Bosnia- } \\
\text { Herzegovina }\end{array}$ & nervous system disorders, sedation & {$[32]$} \\
\hline & erbo moro & Lucca, Italy & against wounds and sprains & [33] \\
\hline & & Mediterranean Area & skin disorders, sore throat in horses & [34] \\
\hline & bar qene & $\begin{array}{l}\text { Albanians, North } \\
\text { Basilicata, Italy }\end{array}$ & diuretic, hemostatic & {$[35,36]$} \\
\hline & crna kopriva & Bosnia-Herzegovina & hysteria & [37] \\
\hline & & Jadovnik Mt., Serbia & $\begin{array}{l}\text { remedy for upset stomach, nausea, and vomiting; symptomatic, treatment of } \\
\text { nervous disorders, sleep disorders, coughs, inflammation, gout }\end{array}$ & [38] \\
\hline & malrubio negro & North Spain & insecticides and repellents against fleas & [39] \\
\hline \multirow{4}{*}{$\begin{array}{l}\text { B. nigra } \\
\text { L. subsp. } \\
\text { anatolica }\end{array}$} & leylimkara & Mersin, Turkey & antiseptic for wounds, to treat inflamed sore in armpit or foot & {$[40]$} \\
\hline & elkurtaran & Taurus Mt., Turkey & to treat flatulence and stomach upset & [41] \\
\hline & $\begin{array}{l}\text { pemberenkli, } \\
\text { oğul otu, arı oto }\end{array}$ & Gönen, Turkey & burns, wounds, headache & {$[42]$} \\
\hline & grip otu & Kırklareli, Turkey & cold, flu & [43] \\
\hline \multirow[t]{5}{*}{ O. fruticosa } & & Yemen & anti-paralytic and for eye diseases & {$[44,45]$} \\
\hline & & North Ethiopia & diarrhea & [46] \\
\hline & sasa & Tigray, Ethiopia & repellent of mosquitos & [47] \\
\hline & geram tungut & Central Ethiopia & tonsillitis & {$[48]$} \\
\hline & shakab, sharm & Saudi Arabia & remedy for sun-stroke & [49] \\
\hline
\end{tabular}


- Table 2 Continued

\begin{tabular}{|c|c|c|c|c|}
\hline Species & $\begin{array}{l}\text { Vernacular } \\
\text { names }\end{array}$ & Area & Use & Ref \\
\hline \multirow[t]{9}{*}{ O. integrifolia } & cheindog & Eritrea & against fleas and mosquitos & {$[50]$} \\
\hline & tinjute & Ethiopia & stomach-ache, evil eye, fever & {$[51]$} \\
\hline & tinjute & Ethiopia & repellent of mosquito and house fly, antimalarial & {$[52,53]$} \\
\hline & tinjute & Ethiopia & Type 2 Diabetes Mellitus & {$[54]$} \\
\hline & chiendog & Tigray, Ethiopia & ectoparasites in livestock & {$[55]$} \\
\hline & chiendog & Tigray, Ethiopia & repellent of mosquitos & {$[47]$} \\
\hline & tungut & Central Ethiopia & evil eye & {$[48]$} \\
\hline & & North Ethiopia & vomiting, nausea, diarrhea, dysentery & {$[46]$} \\
\hline & & North Ethiopia & sterilization, ritual custom & {$[56]$} \\
\hline \multirow[t]{11}{*}{ O. limbata } & bui, phut kanda & Northwest Pakistan & treatment of children's gums and for ophthalmia in men, boils, wound, scabies & {$[57-59]$} \\
\hline & pishkand & Battagram, Pakistan & jaundice & {$[60,61]$} \\
\hline & spin azghay & Malakand, Pakistan & dental problems, wounds, cuts, narcotic, tonic, anticancer and goiter & {$[62]$} \\
\hline & bui & Punjab, Pakistan & treatment of children's gums and for opthalmia in man & {$[63]$} \\
\hline & sassa & Chon. Karak, Pakistan & treatment of children's gums and for opthalmia in man & {$[61]$} \\
\hline & chitta jand & Jhelum, Pakistan & acidity & {$[64]$} \\
\hline & chittakanda & $\begin{array}{l}\text { Azad Jammu and Kashmir, } \\
\text { Pakistan }\end{array}$ & antiseptic, antibacterial, wound healing, ophthalmia, gum diseases & {$[65]$} \\
\hline & spin azghay & Dir lower, Pakistan & hypertension & {$[66]$} \\
\hline & jand & Azad Kashmir, Pakistan & used to improve eye vision & {$[67]$} \\
\hline & & $\begin{array}{l}\text { Abottabad, Cherat, } \\
\text { Mardan, Malakand, Kohat, } \\
\text { Pakistan }\end{array}$ & antiulcer, antispasmodic, antidepressant, opthalmia and gums diseases & {$[68]$} \\
\hline & koribooti & Himalaya & wound healing & [69] \\
\hline O. persica & golder & Baluchistan, Iran & $\begin{array}{l}\text { diabetes, rheumatism, cardiac distress, reducing palpitation, hypertension, } \\
\text { laxative, carminative, antipyretic, cold, hyper lipidemia, gastric discomfort, } \\
\text { parasite repellent, sedative, headache }\end{array}$ & {$[17]$} \\
\hline O. tomentosa & & North Ethiopia & ascariasis, diarrhea & {$[46]$} \\
\hline
\end{tabular}

\section{Phytochemicals}

\section{Diterpenoids}

Seventy-five diterpenes ( $\bullet$ Figs. 1-3) were isolated and characterized both by their AP and roots of taxa of genus Ballota and Otostegia, and their presence is summarized in $>$ Table 3 (labdane diterpenoids) and $>$ Table 4 (other diterpenoids). Apart from $7 \alpha$ acetoxyroyleanone (73) and coleon A (74), belonging to abietane diterpenoids, and 7,8 $\beta$-epoxymomilactone-A (75), belonging to pimarane diterpenoids, 3 main carbon-skeletons occur: labdane, hispanane, and clerodane.

The labdane diterpenoids (1-50) ( $\mathbf{1}$ Figs. 1 and 2 ) are characterized by some interesting structural features. They all belong to a normal labdane stereochemical series, although Gray et al. [118] claimed, based on the optical rotation, that compound 27 had an ent-labdane skeleton. The C-11-C-16 fragment, never carrying an oxygenated function on $\mathrm{C}-11$ and $\mathrm{C}-12$, can occur with different substructures. The most common one involves C-13/C-16 in a furane ring that, in a few cases, is oxidized to $\gamma$-lactone $(6,7,13-16$, $32,49)$. In almost all of the remaining labdanes, C-13 is involved in the formation of a spiro structure including C-9. By NOE correlations between $\mathrm{H}-16$ and $\mathrm{Me}-17$, it has been shown that all of them belong to the $13 R$ stereochemical series.

The decalin moiety contains some constant functional features: the decalin junction is always trans and the methyl groups (C-17) in position C-8; when it is not present, a C-8/C-9 double bond is always $\alpha$-orientated. In the majority of the structures, $\mathrm{C}$ 6 and C-7 show oxygenated functions and methyl 18 is devoid of functionalization.

Hispanane-type diterpenoids ( $\triangleright$ Fig. 3 ) are a scarce group of natural diterpenoids that exhibits a $6 / 7 / 6$ tricylic system featured with a 7-membered carbon ring.

To our knowledge, apart from hispaninic acid (51) and hispanonic acid (52), isolated from $B$. hispanica $[104,105]$, limbatenolides A (54), B (55), D (57), E (58) [107, 109], and limbetazulone (53) [106], isolated from $O$. limbata and limbatenolide $C$ (56), isolated both from O. limbata [107] and O. persica [108], only 5 other natural hispananes have been characterized up until now: methyl verticoate (from Sciadopitys verticillata (Thunb.) Seibold \& Zucc.) [119], salviatalin A [120], salviadigitoside A [120] and salviatalin A 19-O- $\beta$-glucoside [121] (from Salvia digitaloides Diels), and 


\section{(1)}

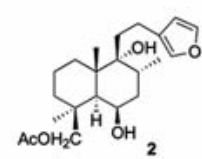

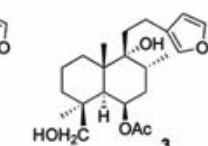

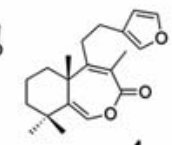

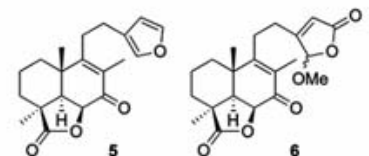

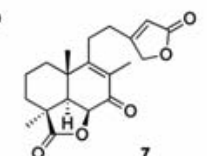

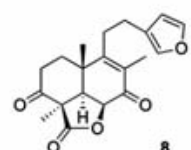

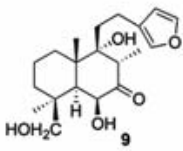

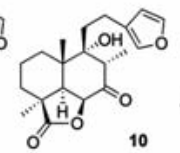

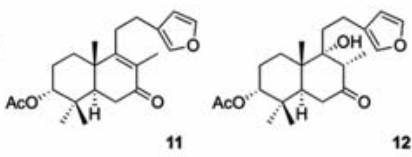

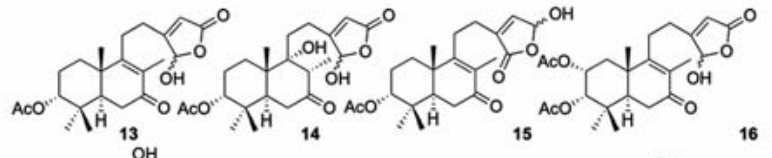
$\mathrm{OH}$

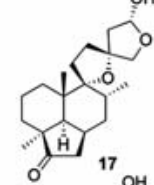

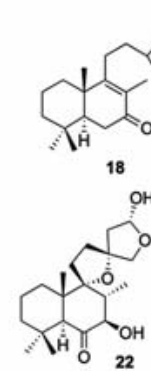

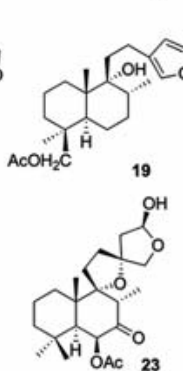

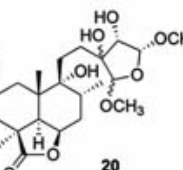<smiles>C1CC2CCC3(CC2C1)OCCO3</smiles><smiles>C[C+]1CC(C)C1</smiles><smiles>CCCCC1C(C)CCC12CCCC2</smiles>

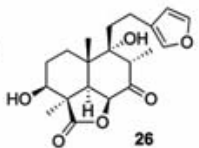

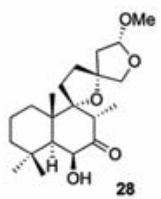

- Fig. 1 Structures of ladbane diterpenes.

viburnumoside (from Viburnum cylindricum Buchanan-Hamilton ex D. Don) [122].

With the exception of viburnumoside, shown to have an ent absolute configuration ( $\beta$ - $\mathrm{H} 5$ and $\alpha-\mathrm{CH}_{3}$ 20) [122], the absolute configurations of any of the other hispananes have been not determined. In this review, we will report the configuration depicted in the original papers ( $\alpha-\mathrm{H} 5$ and $\beta-\mathrm{CH}_{3} 20$ ). The plausible biosynthetic pathway was speculated as a pimarane [121] or labdane way [123].

Clerodane diterpenods ( $\bullet$ Fig. 3 ) showed, differently from labdanes, both a trans junction and a cis junction of the decalin moiety (64-66). Apart from ballodiolic acid (62) and ballodiolic acid A (63), all the other compounds showed the $\mathrm{C}-13 / \mathrm{C}-16$ as furane ring $(67,68)$ or $y$-lactone. Common features to all compounds are the absence of functionalizations at C-1, C-2, C-7, C-11, C12, Me-17, Me-19, and Me-20 and the presence of a carboxylic acid on C-18 that, in some cases, is lactonized with the hydroxyl group on C-6 $(59,61,63,64,67-70)$. In $>$ Table 5 , all of the diterpenes are listed, according to their skeleton, in alphabetical order along with their ${ }^{13} \mathrm{C}$ NMR spectra, when available.

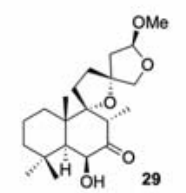

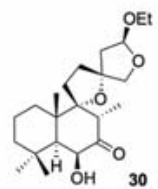

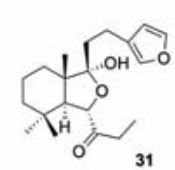

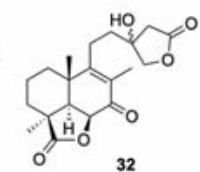

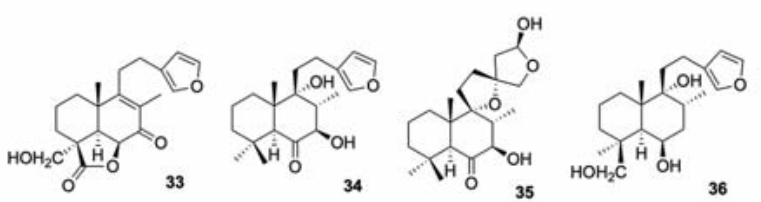

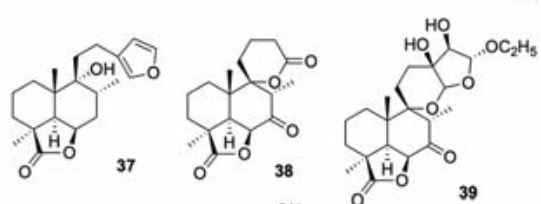

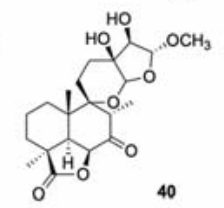

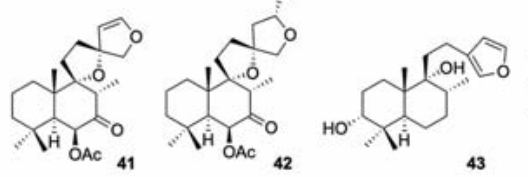

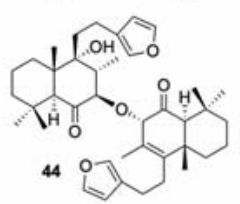

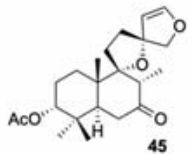

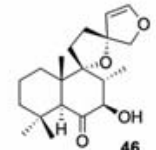

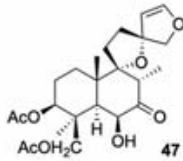

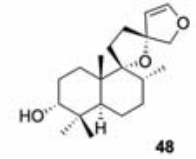

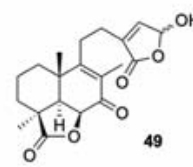<smiles>CC1CCC2CCCCC2C1O</smiles>

Fig. 2 Structures of ladbane diterpenes.

\section{Flavonoids}

In the extensive bibliographic search undertaken, a total of 91 different flavonoids were identified from 22 taxa belonging to Ballota genus and 3 taxa belonging to Otostegia genus.

The structures of the sugar and acyl groups occurring in the secondary metabolites are shown in $\boldsymbol{\nabla}$ Fig. 4 and the formula of all compounds are depicted in $>$ Figs. 5-10. The reported compounds encompass flavones (23 compounds; $>$ Fig. 5), flavonols (13 compounds; - Fig. 6), flavonoid glycosides (24 compounds; - Fig. 7) flavonoid acyl derivatives (20 compounds; > Fig. 8), Cglycosyl-flavonoids (4 compounds; • Fig. 9), flavanones derivatives (4 compounds; > Fig. 10), and flavanols (4 compounds; - Fig. 10).

- Tables 6-8 contain all the flavonoids with their semi-systematic or trivial names, and the genera and species, ordered alphabetically, from which the compounds have been isolated. The most common compounds are apigenin-7-O- $\beta$-D-glucopyranoside (112) (9 taxa), ladanein (79) (8 taxa), apigenin (75) (6 taxa), luteolin-7-O- $\beta$-D-glucopyranoside (116) (6 taxa), and rutin (124) (6 taxa). 


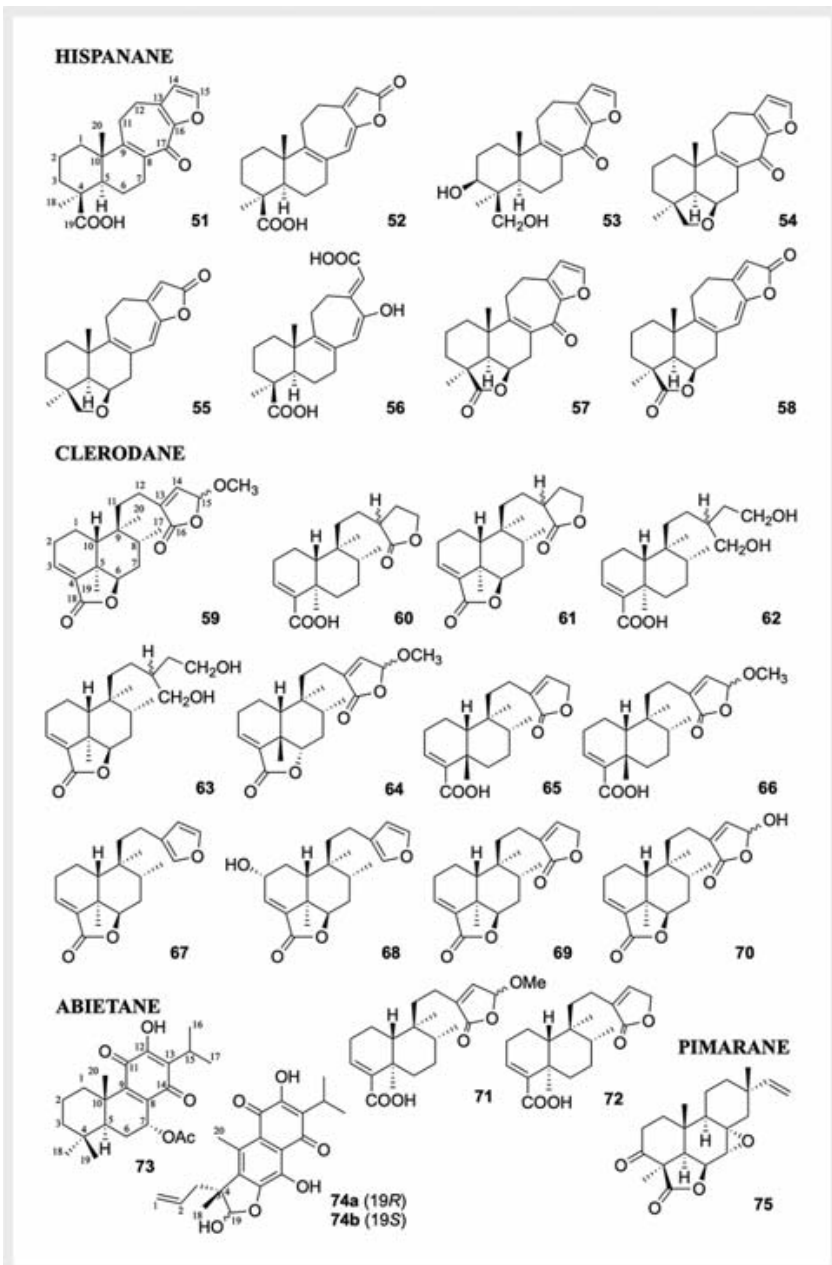

Fig. 3 Structures of hispanane, clerodane, abietane, and pimarane diterpenes.

Flavonoid glycosides, described in 24 reports, account for the vast majority of the 91 total flavonoid reports published so far, followed by flavones (23 reports). Flavonoid coumaroyl glycosides, present in 18 reports (compounds 136-141, 143-150, 152$155,163)$, have a peculiar chemotaxonomical significance and are generally considered valuable markers in the Labiatae family [137].

Although the majority of the flavonoids identified had already been detected in other genera of several families, some were identified for the first time.

From the AP of Ballota acetabulosa, 5 flavonoids were isolated $(112,120,138,145$, and 146). Compound 146 is a new natural flavonoid characterized as the cis isomer of chrysoeriol-7-O- $\beta$ ( $3^{\prime \prime}$-p-coumaroyl)glucopyranoside. The trans isomer 145 (co-occurring in the same species) was previously described from other species belonging to the Lamiaceae family [161].

The new flavonoid coumaroyl glucosides leufolins A (163) and $B$ (152) were isolated from the EtOAc soluble fraction of the WPs of L. urticifolia (syn. B. arabica). Their structures were elucidated on the basis of extensive analysis of 1D and 2D NMR spectral data. Both compounds exhibited significant inhibitory potential against the enzyme butyrylcholinesterase. The unusual oxygenated pattern of the flavonoid moiety of leufolin B (153), devoid of hydroxyl group at C-5 [171], is noteworthy.

From the $n-\mathrm{BuOH}$ extract of the AP of Panzeria alaschanica Kuprian. (syn. B. lanata L.), 2 new flavone C-glycosides, named panzeroside $A$ (156) and $B$ (157), were isolated. The 2 new compounds demonstrated significant and dose-dependent analgesic and anti-inflammatory effects [173].

The $\mathrm{MeOH}$ extract of the roots of $O$. limbata was subjected to several chromatographic separations to give 4 new poly-glycosyl derivatives of kaempferol: compounds 135, 153, 154, and 155, the last 3 also carrying $p$-coumaroyl groups. Their rather complex structures were elucidated by extensive 1D and 2D NMR [167, 172].

\section{Other metabolites}

Apart from diterpenoids and flavonoids, several other metabolites have been identified in Ballota and Otostegia taxa: tritepenoids, steroids ( $\triangleright$ Fig. 11), carboxylic acids ( $\triangleright$ Fig. 12), carotenoids ( $\triangleright$ Fig. 13, Table 9), nitrogen containing compounds ( $\bullet$ Fig. 13), phenylpropanoids, and miscellaneous ( $\bullet$ Fig. 14, Table 10).

The first study on Ballota and Otostegia genera was carried out in 1911 by Piault [197], which isolated the tetrasaccharide stachyose from the roots of $B$. nigra subsp. foetida (237). The following report dates 1934 when, from the same species, Balansard isolated choline (213) and stachydrine (217) [191].

Phenylpropanoids (218-227) occur within 10 compounds and, apart from forsythoside B (223) and verbascoside (227), are present in several species. The main source of this class of compounds is $B$. nigra from which ballotetroside (221), a new derivative, was isolated [194].

Triterpenoids ( 10 compounds) and steroids (8 compounds) are represented by rather common metabolites, although there are some exceptions. For example, moronic acid (173) was isolated for the first time in $B$. cinerea [178], and the new steroid leucisterol (179), as well as the new peroxy acid urticic acid (206), were isolated from the chloroform soluble fraction of the WP of $B$. arabica (syn. L. urticifolia). Leucisterol (179) showed potent inhibitory activity against butyrylcholinesterase enzyme [181]. Recently, the new, structurally quite complex, bacteriohopane-type derivative 178 was isolated from B. cinerea (syn. Roylea cinerea (D. Don) Baill.) [182]. In the same work, the $\beta$-lactam cinerealactam $\mathrm{E}$ (214) [88] was also detected. Both compounds were shown to have a significant effect on the decline in blood glucose levels supporting the role of $B$. cinerea in Ayurvedic medicine for diabetes.

In $>$ Table 11, the occurrence of all of the metabolites in the single taxa is summarized. For some common compounds, whose structures have not been depicted in this review, the trivial name is reported.

\section{EOs}

The chemical composition of EOs obtained from 21 species among Ballota and Otostegia taxa has been investigated. They are mainly distributed in the Mediterranean area, whereas the Otostegia species are almost totally distributed in western Asia and Ballota lanata, syn. Panzeria (Panzerina) lanata, found in eastern Asia (Siberia and Mongolia). The major compounds (>3\%) occurring 
- Table 3 Distribution of labdane diterpenes in Ballota and Otostegia taxa.

\begin{tabular}{|c|c|}
\hline No & Names \\
\hline 1 & $7 \alpha$-acetoxymarrubiin \\
\hline 2 & 6-acetyl-marrubenol \\
\hline 3 & 19-acetyl-marrubenol \\
\hline 4 & balloaucherolide \\
\hline 5 & ballonigrin \\
\hline 6 & ballonigrin lactone $\mathrm{A}$ \\
\hline 7 & ballonigrin lactone B \\
\hline 8 & ballonigrinone \\
\hline 9 & ballotenol \\
\hline 10 & ballotinone \\
\hline 11 & calyenone \\
\hline 12 & calyone \\
\hline 13 & cinereanoid $\mathrm{A}$ \\
\hline 14 & cinereanoid B \\
\hline 15 & cinereanoid C \\
\hline 16 & cinereanoid D \\
\hline 17 & cyllenin A \\
\hline 18 & dehydrohispanolone (hispanone) \\
\hline 19 & 6-dehydroxy-19-acetyl-marrubenol \\
\hline 20 & desertin \\
\hline 21 & 15-epi-cyllenin A \\
\hline 22 & 15-epi-leopersin C \\
\hline 23 & 15-epi-otostegin B \\
\hline 24 & 16-epoxy-9-hydroxylabda-13(16), 14-diene \\
\hline 25 & hispanolone \\
\hline 26 & $3 \beta$-hydroxyballotinone \\
\hline 27 & $6 \beta$-hydroxy-15,16-epoxy-labda-8,13(16),14-trien-7-one \\
\hline 28 & $6 \beta$-hydroxy- $15 \alpha$-methoxy- $9 \alpha, 13,15,16$-bis-epoxylabd-7-one \\
\hline 29 & $6 \beta$-hydroxy-15 $\beta$-methoxy- $9 \alpha, 13,15,16$-bis-epoxylabd-7-one \\
\hline 30 & $6 \beta$-hydroxy- $15 \beta$-ethoxy- $9 \alpha, 13,15,16$-bis-epoxylabd-7-one \\
\hline 31 & $9 \alpha$-hydroxy-6,9:15,16-diepoxy-13(16),14-labdadien-7-one \\
\hline 32 & 13-hydroxyballonigrolide \\
\hline 33 & 18-hydroxyballonigrin \\
\hline 34 & leoheterin \\
\hline 35 & leopersin C \\
\hline 36 & marrubenol \\
\hline 37 & marrubiin \\
\hline 38 & marrulactone \\
\hline 39 & marrulibacétal \\
\hline 40 & marrulibacétal A \\
\hline
\end{tabular}

\section{Taxa}

B. nigra [70]

B. deserti [24]

B. deserti [24]

B. aucheri [71]

B. acetabulosa, B. antalyensis, B. cristata, B. larendana, B. saxatilis subsp. brachyodonta [72, 73], B. aucheri [74], B. inaequidens [72, 73, 75, 76], B. lanata [77],

B. nigra [70], B. nigra subsp. foetida [72,73, 78], B. pseudodictamnus [79],

B. rupestris [70, 78], B. saxatilis [72, 73, 80], B. undulata [81], O. fruticosa [82]

O. limbata [83]

O. limbata [83]

B. rupestris [70,78], B. undulata [81]

B. nigra subsp. foetida [84]

B. aucheri [71], B. nigra subsp. foetida [85], B. undulata [81]

B. cinerea [86]

B. cinerea $[86,87]$

B. cinerea [87]

B. cinerea [87]

B. cinerea [88]

B. cinerea [88]

B. deserti $[89,90]$

B. acetabulosa, B. antalyensis, B. cristata, B. larendana, B. latibracteolata, B macrodonta, $B$. nigra subsp. uncinata, $B$. pseudodictamnus subsp. lycia, B. rotundifolia, B. saxatilis subsp. brachyodonta [72,73], B. saxatilis [72, 73, 80], B. undulata [81], O. fruticosa [82]

B. deserti [24]

B. deserti $[89,90]$

B. deserti $[89,90]$

O. fruticosa [82]

O. fruticosa [82]

B. deserti [24]

B. acetabulosa, B. cristata, B. pseudodictamnus subsp. lycia, B. rotundifolia, B. saxatilis subsp. brachyodonta [72,73], B. africana [91], B. andreuzziana [79], B. hirsuta [92], B. inaequidens $[72,73,75,76]$, B. saxatilis $[72,73,80]$

B. undulata [81]

B. aucheri [74]

B. aucheri [71]

B. aucheri [71]

B. aucheri [71]

B. aucheri [74]

B. lanata [77], B. nigra [93, 94]

B. acetabulosa [95], B. pseudodictamnus [79], B. saxatilis [96]

B. aucheri $[71,74,97]$, O. fruticosa $[82,98]$

O. fruticosa [82]

B. pseudodictamnus [79]

B. deserti $[89,90]$, B. nigra subsp. foetida $[85,99]$

B. deserti $[89,90]$

B. deserti $[89,90]$

B. deserti [90] 
- Table 3 Continued

\begin{tabular}{|l|l|l|}
\hline $\mathbf{N o}$ & Names & Taxa \\
\hline $\mathbf{4 1}$ & otostegin A & O. fruticosa [82] \\
\hline $\mathbf{4 2}$ & otostegin B & O. fruticosa [82] \\
\hline $\mathbf{4 3}$ & otostegindiol & O. integrifolia [100, 101] \\
\hline $\mathbf{4 4}$ & persianone & B. aucheri [74] \\
\hline $\mathbf{4 5}$ & precalyone & B. cinerea [86] \\
\hline $\mathbf{4 6}$ & preleoheterin & B. aucheri [71, 97], O. fruticosa [82] \\
\hline $\mathbf{4 7}$ & preleosibirin & B. nigra subsp. foetida [102] \\
\hline $\mathbf{4 8}$ & preotostegindiol & O. integrifolia [100] \\
\hline $\mathbf{4 9}$ & rupestralic acid & B. rupestris [103] \\
\hline $\mathbf{5 0}$ & vulgarol & O. fruticosa [82] \\
\hline
\end{tabular}

in the chemical composition of the EOs are reported in $~-$ Table 12.

All papers are quite recent, they have been published starting in 2002, except for one [224], published in 1995.

The first published paper concerns the analysis of an Egyptian O. fruticosa [224] species, cultivated in the station of faculty of agriculture of Mansoura University, containing mainly monoterpenes with a high level of thymol (43.7\%). A recent re-investigation of the same species collected wild in the Sinai region showed a composition strongly dominated by sesquiterpenes with the caryophyllene oxide being the most abundant component (60.8\%) [175].

For the single species Ballota sechmenii Gemici \& Leblebici, only the relative content of linalool (5.0\%) and its enantiomeric composition, (+)-isomer (26.9\%), (-)-isomer (73.1\%) have been determined [223]. No other component of the EO was reported.

With only a few exceptions, such as B. lanata [29,206] and 2 B. nigra specimens collected in the Golestan region of Iran [210] and Ukraine [211], respectively, Ballota species EOs are mainly composed of sesquiterpenes with caryophyllene, caryophyllene oxide, and germacrene D often identified as main compounds. On the contrary, in $B$. lanata, monoterpenes are the dominant components, similar to the $B$. nigra of Golestan. The analysis of EOs of different plant parts of Ukrainian B. nigra, showed that fatty acids are the most relevant compounds with sesquiterpenes occurring only in the corallas. However, a nonconventional method of EO extraction was applied.

The Otostegia species studied to date shows controversial results. The case of the $O$. fruticosa, discussed above, and of the O. michauxii, collected in 2 different locations, are emblematic. In fact, the O. michauxii from southern Zagros of Iran show caryophyllene oxide as a main compound [225], whereas the one collected in the Fars province of Iran had an equal amount of monoterpenes and sesquiterpenes [226]. In O. integrifolia, the monoterpene $\alpha$-pinene occurs in $31 \%$ of the oil [56], whereas in some collections of $O$. persica, in several places of southeast Iran, a clear trend cannot be observed.

\section{Biological Properties}

This section deals with the corpus of scientific evidence related to the claims of the biological effects of Ballota and Otostegia genus utilized in traditional medicine ( $\bullet$ Table 2 ). The most widespread usage of the plants is as an aqueous infusion of the drug, which is normally made from the WP dried. The beneficial effects should normally be associated with the presence of polar or water-soluble active principles. Indeed, most of the cited literature deals with the effect of the extract obtained from leaves, stems, roots, flowers, WPs in polar solvent, such as $\mathrm{EtOH}, \mathrm{MeOH}$ and water, as well as mixture of them. However, investigations concerning the bioactivity of fractions obtained with less polar solvents, such as choloroform, $n$-Hex, and EtOAc are also present. In some cases, isolated individual compounds were assessed for their bioactivity. In other cases, the EOs are the focus of the research and their composition is investigated and correlated to the bioactivity observed for that species.

In this report, a selection of the more relevant results, obtained with rigorous and well-defined methodological approaches, are taken into consideration. Redundant investigations that report data concerning the same combinations of plant species and biological targets can often be found in literature, in particular concerning antimicrobial activity.

\section{Antioxidant activity}

Many of the effects of Ballota and Otostegia reported in $>$ Table 2 may be related by their general antioxidant activity, which is well documented in the literature. This activity is generally attributed to the presence of phenolic compounds that are ubiquitous in these genera. In a few cases, terpenoidic compounds, in particular diterpenes, were also identified as the source of antioxidative properties of the drug. The mechanisms of action may include oxygenated radicals scavenging, inhibition of the enzymatic peroxidation, etc. Furthermore, the variety of antioxidant activity evaluation protocols utilized often make it difficult to directly compare the effects of different species. Nevertheless, the antioxidant activity of many Ballota and Otostegia plants has been interestingly and undoubtedly demonstrated. These findings are summarized in $>$ Table 13, where for each investigation reviewed 
the species involved is reported together with its provenience, the extraction mode, and the testing procedure implemented.

As it can be inferred from the analysis of $>$ Table 13, B. nigra and $O$. persica are the most studied species. Their antioxidant activity was evaluated in the EO [196, 228, 229], as well as in different polarity fractions (extraction solvents: $\mathrm{PE}, \mathrm{Ac}, \mathrm{EtOAc}, \mathrm{MeOH}$, $\mathrm{EtOH}$, water) of the extract obtained from leaves, AP, and the WP. In some cases, a number of individual active compounds were isolated and identified. The following were purified from leaf extract (water/EtOH 1:1) of B. nigra collected in France $[236,237]$ : $(+)$-(E)-caffeoyl-L-malic acid (186), verbascoside (227), forsythoside B (223), arenarioside (220), and ballotetroside (221). On the other hand, morin (110) and quercetin (100) were isolated from the bio-guided purification of the methanolic extract of $O$. persica [160]. In an interesting study [236], some chemical indicators of the intracellular inflammatory cascade reaction of the neutrophils were evaluated: superoxide anion, $\mathrm{H}_{2} \mathrm{O}_{2}, \mathrm{HClO}$, and $\mathrm{OH}$ radical. All of the compounds were found to be active, inhibiting the development of oxygenated species to various degrees; only ballotertoside (221) was inactive in the superoxide anion and $\mathrm{OH}$ radical tests. In another study [237], the same authors evaluated all 5 molecules for their inhibitory efficacy with respect to the oxidation of low-density lipoproteins (LDL). They were also assessed for their $\mathrm{Cu}(\mathrm{II})$ chelating power, as this is the well-known mechanism of action of the antioxidant quercetin. None of them were able to behave as a copper chelating agent. The infusion of $B$. nigra leaves from the Czech Republic was shown to possess outstanding antioxidant activity, in particular by DPPH, NO, and superoxide anion scavenging ability [185]. On the contrary, no OH radical scavenging ability was revealed. In terms of organic acids and polyphenol content of the infusion, the composition was determined by HPLC/DAD and HPLC/UV analysis and the authors inferred the correlation between these compounds and the observed antioxidant activity. The crude methanolic extract of AP of B. hirsuta [234] and O. limbata [241] were further partitioned in several solvents in order to select phenolic enriched subfractions following the solubility properties of different compounds, the EtOAc fraction being the most active in both cases: in the DPPH test, the $I_{50}$ were $13.53 \mu \mathrm{g} / \mathrm{mL}$ for $O$. limbata and $70.0 \mu \mathrm{g} / \mathrm{mL}$ for $B$. hirsuta. On the contrary, the less active fraction of the extract was the one in $\mathrm{H}_{2} \mathrm{O}$ for B. nigra $(129.5 \mu \mathrm{g} / \mathrm{mL})$ and the one obtained in $\mathrm{CHCl}_{3}$ for B. hirsuta $(260 \mu \mathrm{g} / \mathrm{mL})$. A significant difference in the antioxidant power of different solvent fractions was found for O. persica collected in Iran [158]. The fraction soluble in $\mathrm{MeOH}$ showed LPO inhibition in the $\mathrm{NH}_{4} \mathrm{SCN}$ test comparable to that of $\alpha$-tocopherol $(95.87 \%)$. On the other hand, the fractions soluble in $n$-Hex and $\mathrm{CHCl}_{3}$ were poorly active (2.5 and $1.9 \%$, respectively). These variations in the antioxidant activity, evidenced by different partition solvents, can be related to the affinity of the active metabolites (poly-phenols and flavonoids) to these solvents.

The relation between the antioxidant power of 0 . persica extract (AP, 70\% MeOH) and the protective effect against the damages caused by the oxidative stress on the endothelium cells, was investigated in vitro on a human cell line: the umbilical vein endothelial cells [244]. No toxicity was revealed up to $250 \mu \mathrm{g} / \mathrm{mL}$ of extract. The oxidative effects were induced by $\mathrm{H}_{2} \mathrm{O}_{2}$ and evaluated by the cell viability essay (MTT), intracellular and extracellular total
- Table 4 Distribution of other diterpenes in Ballota and Otostegia taxa.

\begin{tabular}{|c|c|c|}
\hline & Hispanane skeleton & \\
\hline 51 & hispaninic acid ${ }^{a}$ & B. hispanica $[104,105]$ \\
\hline 52 & hispanonic acida & B. hispanica $[104,105]$ \\
\hline 53 & limbetazulone & O. limbata [106] \\
\hline 54 & limbatenolide A & O. limbata [107] \\
\hline 55 & limbatenolide B & O. limbata [107] \\
\hline 56 & limbatenolide C & O. limbata [107], O. persica [108] \\
\hline 57 & limbatenolide D & O. limbata [109] \\
\hline \multirow[t]{2}{*}{58} & limbatenolide E & O. limbata [109] \\
\hline & Clerodane skeleton & \\
\hline 59 & ballatenolide $\mathrm{A}$ & O. limbata [107], O. persica [108] \\
\hline 60 & ballotenic acid & O. limbata [110] \\
\hline 61 & ballotenic acid $\mathrm{A}$ & O. limbata [111] \\
\hline 62 & ballodiolic acid & O. limbata [110] \\
\hline 63 & ballodiolic acid $\mathrm{A}$ & O. limbata [111] \\
\hline 64 & limbatolide A & O. limbata [112] \\
\hline 65 & limbatolide B & O. limbata [112] \\
\hline 66 & limbatolide C & O. limbata [112] \\
\hline 67 & limbatolide D & O. limbata [113] \\
\hline 68 & limbatolide E & O. limbata [113] \\
\hline 69 & limbatolide F & O. limbata [114] \\
\hline 70 & limbatolide G & O. limbata [114] \\
\hline 71 & 15-methoxypatagonic acid & O. limbata [107], O. persica [108] \\
\hline \multirow[t]{2}{*}{72} & patagonic acid & O. limbata [107], O. persica [108] \\
\hline & Abietane skeleton & \\
\hline 73 & $7 \alpha$-acetoxyroyleanone & B. nigra [115] \\
\hline \multirow[t]{2}{*}{74} & coleon A & B. cinerea $[116]$ \\
\hline & Pimarane skeleton & \\
\hline 75 & 7,8 $\beta$-epoxymomilactone-A & B. arabica [117] \\
\hline
\end{tabular}

peroxides test, and FRAP. The extract significantly reduced the effect of $\mathrm{H}_{2} \mathrm{O}_{2}$ in a dose-dependent fashion $(50-250 \mu \mathrm{g} / \mathrm{mL})$.

The direct correlation between the phenolic compounds content is rather general, although some exceptions are also known. For example, in an investigation of the antioxidant properties of O. limbata from Pakistan [242], the methanolic extract was subsequently divided in fractions soluble in $n$-Hex, $\mathrm{CHCl}_{3}$, EtOAc, $n$ $\mathrm{BuOH}, \mathrm{MeOH}$, and $\mathrm{H}_{2} \mathrm{O}$, and total content in phenolic compounds and flavonoids was determined. The EtOAc fraction resulted the one with the highest antioxidant power $\left(\mathrm{EC}_{50} \mathrm{TPPH}\right.$ test $60.9 \mu \mathrm{g}$, total phenolic compounds $1119 \mathrm{mg}$ ), even if the higher phenols content was found in $n$-Hex $\left(3908 \mathrm{mg}, \mathrm{EC}_{50} 226.1 \mu \mathrm{g}\right)$ and 1-butanol (3037 mg, EC50 $96.3 \mu \mathrm{g})$.

The antioxidant activity of apigenin-7-O-(6"-O-[E]-coumaroyl)$\beta$-glucopyranoside (139), a flavonoid glycoside isolated from the B. lanata (syn. P. alaschanica) AP collected in China [169], was determined in vivo by evaluating its lipid peroxidation inhibitory activity. A diabetes mellitus-related oxidative stress was induced in 


\begin{tabular}{|c|c|c|c|c|c|c|c|c|c|c|c|c|c|c|c|c|c|c|c|c|c|c|c|c|}
\hline ֻँّ & & 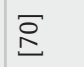 & & & $\bar{\Xi}$ & $\underline{\bar{\infty}}$ & $\underset{\stackrel{\infty}{\infty}}{\underline{\infty}}$ & $\underset{\underline{\infty}}{\bar{\infty}}$ & $\underline{\bar{\Phi}}$ & $\begin{array}{l}\bar{\Phi} \\
\underline{\infty}\end{array}$ & $\underline{\bar{\infty}}$ & $\begin{array}{l}\bar{D} \\
\stackrel{\infty}{ }\end{array}$ & $\underset{\underline{\infty}}{\bar{\infty}}$ & $\underset{\infty}{\mp}$ & $\underset{\infty}{\widetilde{\infty}}$ & 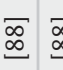 & $\stackrel{\infty}{\infty} \underset{\infty}{\infty}$ & $\stackrel{\mathbb{J}}{\Xi}$ & $\stackrel{\text { In }}{\Xi}$ & $\stackrel{\bar{\Xi}}{\mathbb{I}}$ & $\begin{array}{l}\Phi \\
\mathscr{\infty}\end{array}$ & $\stackrel{\mathbb{I}}{\Xi}$ & 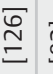 & $\underset{\infty}{\infty}$ \\
\hline $\bar{N}$ & & $\stackrel{\circ}{\dot{\sim}}$ & & & & & & & & & & $\overline{\bar{N}}$ & $\stackrel{\stackrel{n}{\sim}}{\sim}$ & $\check{\bar{v}}$ & $\overline{\dot{N}}$ & $\overline{\dot{N}} \overline{7}$ & $\overline{\dot{v}}$ & & & $\stackrel{\text { }}{\text { iे }}$ & ถู่ & & & 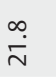 \\
\hline$=$ & & $\stackrel{\circ}{\stackrel{ }{\Sigma}}$ & & & & & 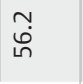 & & & & & $\begin{array}{l} \\
\stackrel{1}{\Omega}\end{array}$ & $\begin{array}{l}0 \\
\stackrel{0}{+} \\
\stackrel{2}{2}\end{array}$ & 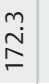 & $\begin{array}{l}\stackrel{0}{\mathbf{N}} \\
\stackrel{-}{2}\end{array}$ & 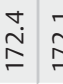 & $\overline{\underline{N}}$ & & & 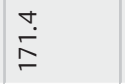 & 苞 & & & : \\
\hline กิ & & $\stackrel{\circ}{\underset{\sim}{\sim}}$ & & & $\begin{array}{l}\stackrel{\varphi}{\sim} \\
\stackrel{\sim}{\sim}\end{array}$ & $\begin{array}{l}\dot{0} \\
\stackrel{\infty}{\sim}\end{array}$ & $\stackrel{\grave{\sim}}{\stackrel{\sim}{\mathbf{N}}}$ & $\overline{\tilde{\sim}}$ & $\stackrel{\stackrel{\sim}{d}}{\sim}$ & $\bar{\sim}$ & $\stackrel{\stackrel{9}{\rightleftharpoons}}{\stackrel{2}{r}}$ & 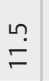 & $\begin{array}{l}\stackrel{\leftrightarrow}{\varphi} \\
\stackrel{\circ}{ }\end{array}$ & 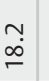 & $\begin{array}{l}\stackrel{\infty}{0} \\
\stackrel{\varphi}{\circ}\end{array}$ & 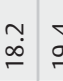 & 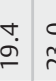 & ì & $\stackrel{-\infty}{\infty}$ & $\stackrel{9}{\circ}$ & $\stackrel{\sim}{\underset{N}{N}}$ & $\underset{\sim}{\sim}$ & $\stackrel{\vec{g}}{\sigma}$ & $\overbrace{}^{n}$ \\
\hline ㅇ & & $\stackrel{\check{\infty}}{\stackrel{\check{\infty}}{\rightleftharpoons}}$ & & & 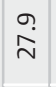 & $\begin{array}{l}\overline{0} \\
\infty\end{array}$ & 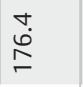 & 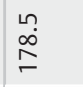 & 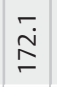 & ㅇ.ㅁ & $\begin{array}{l}m \\
\stackrel{m}{\infty} \\
\stackrel{-}{-}\end{array}$ & $\stackrel{m}{\stackrel{m}{N}}$ & $\stackrel{\stackrel{\sim}{\sim}}{\stackrel{N}{*}}$ & $\stackrel{\grave{n}}{\dot{N}}$ & $\stackrel{q}{\dot{N}}$ & $\stackrel{\infty}{\stackrel{\infty}{N}}$ & $\stackrel{\vec{\sim}}{\bar{\sim}} \mathfrak{z}$ & 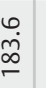 & $\frac{m}{\sim}$ & $\bar{\sigma}$ & 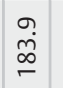 & $\underset{\substack{\infty \\
\infty}}{m}$ & $\underset{m}{\grave{m}}$ & $\stackrel{q}{\dot{d}}$ \\
\hline$\stackrel{\infty}{\stackrel{0}{2}}$ & & $\stackrel{\infty}{\underset{\sim}{\sim}}$ & & & $\stackrel{-\vec{\infty}}{\stackrel{\infty}{\sim}}$ & $\stackrel{\text { Ln }}{\stackrel{\sim}{\sim}}$ & $\hat{\grave{\nu}}$ & $\overline{\grave{N}}$ & $\stackrel{\varphi}{\dot{N}}$ & $\stackrel{\infty}{\stackrel{\infty}{\sim}}$ & $\stackrel{m}{\stackrel{n}{~}}$ & $\stackrel{\circ}{\grave{N}}$ & $\underset{\stackrel{0}{\sim}}{\stackrel{\sim}{\sim}}$ & 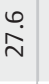 & 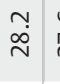 & 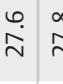 & $\stackrel{\infty}{\stackrel{\infty}{\sim}}$ & 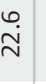 & 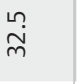 & $\begin{array}{l}\stackrel{\infty}{\grave{\nu}} \\
\stackrel{\omega}{\sim}\end{array}$ & $\stackrel{\circ}{\stackrel{\sim}{\sim}}$ & 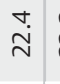 & $\underset{\mathbb{N}}{\mathbb{N}}$ & $\bar{m}$ \\
\hline$\approx$ & & 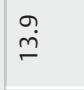 & & & $\begin{array}{l}\stackrel{0}{\rightleftarrows} \\
=\end{array}$ & $\stackrel{g}{\check{C}}$ & 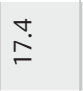 & 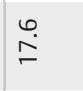 & $\overline{\grave{I}}$ & $\hat{\infty}$ & 亡ُ & $\begin{array}{l}\circ \\
\stackrel{\infty}{\leftarrow}\end{array}$ & $\underset{\infty}{m}$ & $\stackrel{\varphi}{=}$ & $\begin{array}{l}0 \\
\infty\end{array}$ & $\stackrel{\infty}{=}$ & 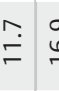 & 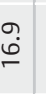 & 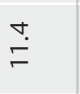 & $\stackrel{\sim}{\varrho}$ & $\begin{array}{l}\stackrel{\infty}{0} \\
\stackrel{\varphi}{\varphi}\end{array}$ & $\begin{array}{l}\sigma \\
\varrho \\
\varrho\end{array}$ & $\stackrel{m}{m}$ & $\stackrel{n}{\sigma}$ \\
\hline 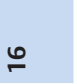 & & 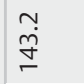 & & & $\underset{\check{g}}{\check{g}}$ & $\begin{array}{l}\infty \\
\infty \\
\stackrel{\infty}{\sim} \\
\stackrel{2}{n}\end{array}$ & $\begin{array}{l}\stackrel{0}{\circ} \\
\stackrel{2}{ }\end{array}$ & $\stackrel{\bar{N}}{\underline{I}}$ & $\begin{array}{l}\infty \\
\infty \\
\stackrel{\infty}{n} \\
\end{array}$ & $\begin{array}{l}\infty \\
\infty \\
\stackrel{\infty}{\sim} \\
\stackrel{2}{n}\end{array}$ & $\begin{array}{l}\hat{\infty} \\
\stackrel{0}{m} \\
\stackrel{n}{n}\end{array}$ & $\begin{array}{l}0 \\
\infty \\
\stackrel{m}{n} \\
m\end{array}$ & 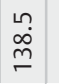 & $\stackrel{\circ}{\circ}$ & 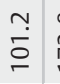 & 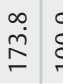 & 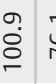 & $\overline{\dot{\rho}}$ & $\begin{array}{l}\stackrel{0}{\infty} \\
\stackrel{\infty}{m} \\
\end{array}$ & $\begin{array}{l}\stackrel{+}{\infty} \\
\stackrel{\infty}{\sim}\end{array}$ & $\begin{array}{l}0 \\
\stackrel{0}{0} \\
\stackrel{0}{\circ}\end{array}$ & ஜ̊̀ & $\underset{\infty}{\stackrel{\infty}{\infty}}$ & $\stackrel{\stackrel{0}{\Gamma}}{ }$ \\
\hline$\stackrel{n}{\check{2}}$ & & $\begin{array}{l}\infty \\
\infty \\
\stackrel{m}{\longrightarrow}\end{array}$ & & & 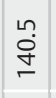 & $\overline{\dot{g}}$ & $\begin{array}{l}\text { g̊ } \\
\stackrel{0}{\circ}\end{array}$ & $\stackrel{+}{\stackrel{+}{\lessgtr}}$ & 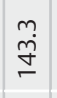 & $\begin{array}{l}\stackrel{m}{g} \\
\stackrel{\tilde{g}}{g}\end{array}$ & $\stackrel{\bar{y}}{\check{\sigma}}$ & 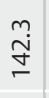 & $\stackrel{\bar{g}}{\check{g}}$ & $\begin{array}{l}\stackrel{0}{\tilde{e}} \\
\stackrel{n}{=}\end{array}$ & $\stackrel{\stackrel{n}{E}}{=}$ & 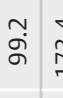 & $\stackrel{+}{\stackrel{丶}{\rightleftarrows}}$ & $\begin{array}{c}\text { a. } \\
\sigma\end{array}$ & $\stackrel{\circ}{\stackrel{\sim}{\Xi}}$ & 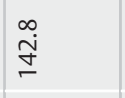 & 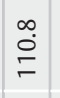 & స్ & ஜ் & $\frac{y}{b}$ \\
\hline$\nabla$ & & $\stackrel{\hat{\rho}}{\rightleftharpoons}$ & & & $\begin{array}{l}\stackrel{\text { L }}{\circ} \\
\stackrel{口}{\rightleftharpoons}\end{array}$ & 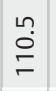 & $\frac{m}{\stackrel{g}{g}}$ & 字 & $\begin{array}{l}\stackrel{n}{n} \\
\stackrel{0}{\rightleftharpoons}\end{array}$ & $\stackrel{\stackrel{+}{\rightleftarrows}}{\rightleftarrows}$ & 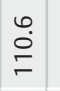 & $\begin{array}{l}\stackrel{n}{n} \\
\stackrel{0}{\rightleftharpoons}\end{array}$ & 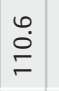 & $\stackrel{\substack{\infty \\
\stackrel{\infty}{\rightleftharpoons}}}{\rightleftharpoons}$ & $\stackrel{\infty}{\stackrel{\infty}{\sim}}$ & 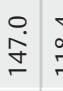 & 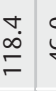 & $\begin{array}{l}0 \\
\dot{y} \\
\dot{y}\end{array}$ & $\begin{array}{l}\stackrel{n}{0} \\
\stackrel{0}{=}\end{array}$ & 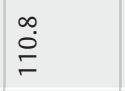 & $\begin{array}{c}\tilde{D} \\
\stackrel{\infty}{0}\end{array}$ & $\overline{\dot{\infty}}$ & $\begin{array}{l}\stackrel{g}{r} \\
\dot{f}\end{array}$ & $\bar{y}$ \\
\hline$\stackrel{m}{m}$ & & $\begin{array}{l}\stackrel{0}{ \pm} \\
\stackrel{+}{\simeq}\end{array}$ & & & 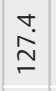 & $\stackrel{\stackrel{\sim}{\tilde{j}}}{\underset{\sim}{\simeq}}$ & Б̄ & 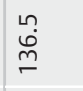 & 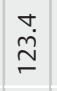 & $\begin{array}{l}\stackrel{0}{\dot{\theta}} \\
\stackrel{\sim}{\simeq}\end{array}$ & 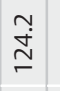 & 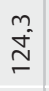 & $\begin{array}{l}\stackrel{\bullet}{ \pm} \\
\stackrel{ \pm}{\simeq}\end{array}$ & 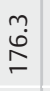 & $\begin{array}{l}\stackrel{0}{\mathbb{N}} \\
\stackrel{1}{\Sigma}\end{array}$ & 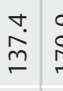 & $\begin{array}{l}0 \\
\stackrel{2}{2} \\
\end{array}$ & $\begin{array}{l}\text { Ln } \\
\text { Ln' } \\
\infty\end{array}$ & 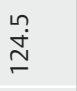 & $\stackrel{\stackrel{n}{\stackrel{n}{n}}}{\simeq}$ & $\stackrel{\stackrel{1}{\infty}}{\infty}$ & $\begin{array}{l}\stackrel{n}{0} \\
\text { 心) }\end{array}$ & $\begin{array}{l}0 \\
\bar{\sigma}\end{array}$ & $\begin{array}{l}\text { هं } \\
\dot{8}\end{array}$ \\
\hline$\simeq$ & & $\stackrel{\nabla}{\infty}$ & & & $\stackrel{\nabla}{\stackrel{D}{~}}$ & $\begin{array}{l}\dot{\dot{d}} \\
\dot{\sim}\end{array}$ & $\stackrel{\bullet}{\stackrel{\sim}{\sim}}$ & $\stackrel{\sigma}{\underset{\sim}{N}}$ & $\check{\dot{\sim}}$ & $\stackrel{\circ}{\underset{\sim}{\mathbf{i}}}$ & $\stackrel{\bar{\infty}}{\stackrel{-}{\infty}}$ & $\stackrel{m}{\stackrel{m}{\sim}}$ & $\stackrel{\stackrel{n}{\sim}}{\stackrel{\sim}{N}}$ & $\stackrel{\bullet}{\stackrel{\sim}{\sim}}$ & $\stackrel{0}{\stackrel{\dot{d}}{\sim}}$ & $\stackrel{\substack{n \\
\sim}}{\sim}$ & 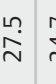 & $\begin{array}{l}\text { fím } \\
\dot{m}\end{array}$ & $\stackrel{\stackrel{\sim}{\sim}}{\sim}$ & $\stackrel{\bullet}{\stackrel{\sim}{\sim}}$ & $\stackrel{\nabla}{\stackrel{\sim}{\sim}}$ & $\underset{m}{\stackrel{N}{s}}$ & $\begin{array}{c}\infty \\
\infty \\
\infty \\
m \\
m\end{array}$ & $\begin{array}{l}\infty \\
\dot{\infty} \\
m \\
m\end{array}$ \\
\hline$=$ & & $\stackrel{+}{\infty} \underset{\sim}{\sim}$ & & & $\stackrel{\infty}{\ddot{\sim}}$ & $\begin{array}{l}\stackrel{0}{\dot{m}} \\
\dot{m}\end{array}$ & शे & $\stackrel{\stackrel{N}{m}}{\bar{m}}$ & $\stackrel{m}{m}$ & $\begin{array}{l}\stackrel{\llcorner}{\sim} \\
\tilde{\gamma}\end{array}$ & $\begin{array}{c}\infty \\
\stackrel{\infty}{m} \\
\stackrel{m}{n}\end{array}$ & வें & $\stackrel{\sim}{\dot{m}}$ & $\stackrel{\infty}{\stackrel{\infty}{\sim}}$ & $\underset{m}{m}$ & $\begin{array}{l}\dot{\omega} \\
\stackrel{\sim}{\sim}\end{array}$ & $\stackrel{n}{\stackrel{n}{\sim}}$ & $\overline{\text { Dे }}$ & ָ̃. & 올 & $\underset{\substack{\sim \\
\sim}}{\sim}$ & $\begin{array}{l}\stackrel{\sigma}{0} \\
\stackrel{\infty}{\sim}\end{array}$ & ִֻ & і் \\
\hline 웅 & & Бें & & & $\begin{array}{l}\infty \\
\ddot{\gamma}\end{array}$ & $\hat{i}$ & $\overline{\text { m் }}$ & $\begin{array}{l}\stackrel{L}{0} \\
\infty \\
m\end{array}$ & $\overline{\dot{m}}$ & $\bar{g}$ & $\begin{array}{l}\stackrel{0}{\dot{q}} \\
\dot{q}\end{array}$ & $\begin{array}{l}\ddot{q} \\
\dot{q}\end{array}$ & $\begin{array}{l}\stackrel{्}{\gamma} \\
\dot{\gamma}\end{array}$ & $\overline{\tilde{g}}$ & 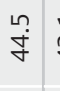 & $\overline{\tilde{g}}$ & 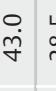 & 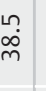 & $\begin{array}{l}\text { ğ } \\
\text { ğ }\end{array}$ & 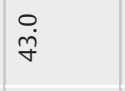 & $\bar{q}$ & $\begin{array}{c}\vec{D} \\
\stackrel{m}{m}\end{array}$ & $\begin{array}{c}m \\
\text { of } \\
\stackrel{f}{q}\end{array}$ & $\begin{array}{l}\overrightarrow{\tilde{\gamma}} \\
\tilde{y}\end{array}$ \\
\hline$a$ & & ָָ & & & $\begin{array}{l}\infty \\
\stackrel{\infty}{\leftrightarrow} \\
\underline{0}\end{array}$ & $\begin{array}{l}\hat{\dot{\theta}} \\
\stackrel{0}{0}\end{array}$ & 孞 & 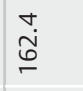 & $\hat{\tilde{\theta}}$ & 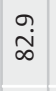 & 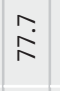 & $\begin{array}{l}\stackrel{0}{0} \\
\dot{m}\end{array}$ & $\begin{array}{l}\stackrel{0}{\infty} \\
\infty\end{array}$ & $\begin{array}{l}m \\
\infty \\
\emptyset \\
\emptyset\end{array}$ & $\underset{\infty}{\stackrel{n}{\sim}}$ & $\begin{array}{ll}\stackrel{n}{n} \\
\stackrel{\infty}{o} \\
\stackrel{0}{0}\end{array}$ & \begin{tabular}{l|l}
$\infty$ & 2 \\
$\stackrel{0}{0}$ & 2
\end{tabular} & $\begin{array}{c}m \\
\bar{a}\end{array}$ & $\stackrel{\dot{0}}{\grave{\varphi}}$ & $\begin{array}{l}\stackrel{0}{r} \\
\stackrel{\nabla}{r}\end{array}$ & 孞 & 吕 & ๙ே. & $\begin{array}{l}N \\
\infty\end{array}$ \\
\hline$\infty$ & & $\begin{array}{l}\infty \\
\infty \\
m \\
m\end{array}$ & & & 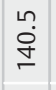 & $\stackrel{\stackrel{v}{m}}{\stackrel{m}{m}}$ & 岕 & $\begin{array}{l}\hat{i} \\
\stackrel{m}{=}\end{array}$ & $\begin{array}{l}\stackrel{0}{\mathfrak{m}} \\
\stackrel{m}{\underline{m}}\end{array}$ & $\begin{array}{l}\stackrel{0}{\dot{b}} \\
\dot{f}\end{array}$ & $\stackrel{+}{i}$ & $\bar{\emptyset}$ & $\stackrel{\text { ㅇ. }}{\text { in }}$ & $\frac{g}{\dot{m}}$ & $\frac{\text { ? }}{\overline{i n}}$ & 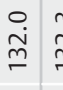 & $\underset{m}{\stackrel{m}{m}}$ & 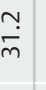 & $\stackrel{m}{m}$ & $\begin{array}{l}\dot{\varphi} \\
\dot{m}\end{array}$ & $\stackrel{\stackrel{\llcorner}{\sim}}{\underset{m}{m}}$ & $\frac{n}{m}$ & $\begin{array}{l}\stackrel{0}{\dot{r}} \\
\dot{f}\end{array}$ & 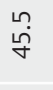 \\
\hline r & & $\begin{array}{l}\stackrel{0}{\circ} \\
\stackrel{\circ}{\circ}\end{array}$ & & & 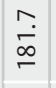 & $\begin{array}{l}\tilde{n} \\
\stackrel{n}{\sigma}\end{array}$ & 悹 & ז. & $\frac{m}{\bar{\sigma}}$ & $\stackrel{\stackrel{\rho}{m}}{\stackrel{m}{\sim}}$ & 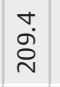 & $\begin{array}{l}m \\
\stackrel{2}{\sigma}\end{array}$ & $\stackrel{+}{\dot{亠}}$ & 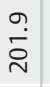 & $\stackrel{\stackrel{n}{+}}{\underset{\sim}{N}}$ & $\begin{array}{l}\stackrel{i}{\mathrm{~d}} \\
\stackrel{\mathrm{N}}{2}\end{array}$ & ma & $\begin{array}{l}\text { ?े } \\
\text { cे }\end{array}$ & ֻ̊̀े & $\frac{n}{m}$ & $\stackrel{\varphi}{\dot{m}}$ & $\begin{array}{l}\text { gे } \\
\dot{m}\end{array}$ & 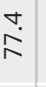 & 㖞 \\
\hline 6 & & $\begin{array}{l}\stackrel{\infty}{\rho} \\
\stackrel{n}{R}\end{array}$ & & & $\overline{\check{g}}$ & 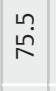 & $\begin{array}{l}\infty \\
\stackrel{\infty}{\wedge}\end{array}$ & $\bar{\infty}$ & $\stackrel{\stackrel{n}{+}}{\stackrel{N}{N}}$ & $\begin{array}{l}\stackrel{g}{+} \\
\stackrel{T}{\sim}\end{array}$ & $\begin{array}{l}\stackrel{0}{\circ} \\
\stackrel{\text { L }}{n}\end{array}$ & $\overline{\dot{m}}$ & $\begin{array}{l}\stackrel{\sim}{n} \\
\infty \\
m\end{array}$ & 望 & 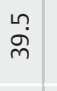 & 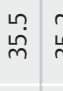 & $\begin{array}{ll}\stackrel{n}{n} \\
\stackrel{n}{n}\end{array}$ & $\begin{array}{l}\hat{n} \\
\text { si }\end{array}$ & 岇 & $\stackrel{b}{\underset{\sim}{\sim}}$ & $\begin{array}{l}m \\
\stackrel{\rho}{n}\end{array}$ & 点 & 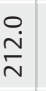 & $\stackrel{\ddot{g}}{i}$ \\
\hline in & & $\begin{array}{l}\stackrel{0}{\dot{f}} \\
\dot{\varphi}\end{array}$ & & & 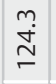 & 年 & ma. & $\begin{array}{c}m \\
\infty \\
\infty\end{array}$ & $\begin{array}{l}\text { No } \\
\text { On }\end{array}$ & $\stackrel{+}{i}$ & $\begin{array}{l}\dot{\leftrightarrow} \\
\dot{J}\end{array}$ & $\stackrel{\text { Ln }}{\dot{f}}$ & $\overline{\dot{y}}$ & 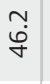 & $\underset{\mathscr{F}}{\stackrel{M}{H}}$ & $\begin{array}{l}\tilde{y} \\
\text { gu }\end{array}$ & 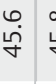 & 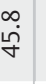 & $\begin{array}{l}\text { No } \\
\text { in }\end{array}$ & 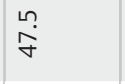 & $\begin{array}{l}\stackrel{\circ}{\dot{f}} \\
\dot{f}\end{array}$ & $\begin{array}{l}\infty \\
\dot{f} \\
\dot{f}\end{array}$ & \begin{tabular}{c}
\multirow{2}{n}{} \\
ñ
\end{tabular} & $\bar{g}$ \\
\hline$\nabla$ & & $\hat{\tilde{\gamma}}$ & & & 空 & 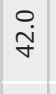 & $\stackrel{\widetilde{Y}}{\tilde{F}}$ & $\begin{array}{l}9 \\
\text { घे }\end{array}$ & $\overline{\mathrm{N}}$ & $\stackrel{\sim}{\stackrel{7}{*}}$ & $\begin{array}{l}\stackrel{\varphi}{\dot{\sigma}} \\
\dot{\sigma}\end{array}$ & $\begin{array}{l}\dot{\varphi} \\
\dot{m}\end{array}$ & $\begin{array}{l}\stackrel{0}{1} \\
\text { m }\end{array}$ & $\stackrel{\text { }}{\stackrel{m}{m}}$ & $\overline{\dot{m}}$ & $\stackrel{r}{\stackrel{m}{m}}$ & $\begin{array}{cc}\stackrel{a}{a} \\
\infty \\
m\end{array}$ & 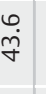 & $\bar{m}$ & $\begin{array}{l}\text { gे } \\
\text { pi }\end{array}$ & $\begin{array}{l}\stackrel{\sigma}{r} \\
\tilde{\gamma}\end{array}$ & $\begin{array}{l}\stackrel{\varphi}{\dot{\gamma}} \\
\dot{\gamma}\end{array}$ & $\underset{\mathrm{m}}{\stackrel{\dot{m}}{\mathrm{~d}}}$ & 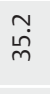 \\
\hline$m$ & & & 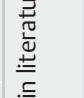 & 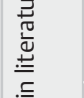 & $\underset{m}{\stackrel{m}{m}}$ & 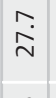 & $\stackrel{\bullet}{\stackrel{0}{\sim}}$ & $\stackrel{\sim}{\stackrel{\sim}{\infty}} \underset{\sim}{\sim}$ & $\stackrel{\nabla}{\stackrel{\sim}{\sim}}$ & $\stackrel{m}{\stackrel{m}{m}}$ & $\begin{array}{l}\infty \\
\infty \\
\infty \\
\sim\end{array}$ & 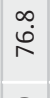 & 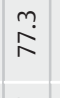 & $\stackrel{\substack{\infty \\
\infty}}{\wedge}$ & ָू & $\stackrel{\substack{n \\
\infty}}{\infty}$ & 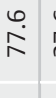 & 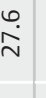 & $\frac{m}{\sigma}$ & 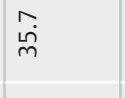 & $\stackrel{\substack{\infty \\
\sim}}{\sim}$ & $\stackrel{\grave{i}}{\sim}$ & 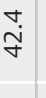 & $\overline{\dot{J}}$ \\
\hline$N$ & & & 竞 & 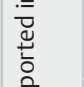 & $\stackrel{\stackrel{n}{\sim}}{\check{r}}$ & $\stackrel{\infty}{\check{1}}$ & $\frac{9}{\bar{N}}$ & $\stackrel{m}{\grave{N}}$ & 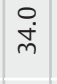 & $\begin{array}{l}\stackrel{\sigma}{\infty} \\
\infty \\
-\end{array}$ & $\stackrel{\infty}{\stackrel{丶}{\rightleftharpoons}}$ & 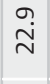 & $\hat{\grave{N}}$ & $\begin{array}{l}\stackrel{\infty}{\sim} \\
\underset{\sim}{\nu}\end{array}$ & $\stackrel{\infty}{\underset{\sim}{\sim}}$ & $\stackrel{\infty}{\tilde{\sim}}$ & స̃. & $\stackrel{m}{\stackrel{m}{=}}$ & \begin{tabular}{l}
$0^{\infty}$ \\
\hdashline
\end{tabular} & $\stackrel{\sim}{\infty}$ & 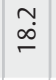 & 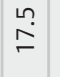 & 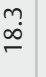 & $\bar{g}$ \\
\hline- & & $\stackrel{\nabla}{m}$ & $\begin{array}{l}\frac{\overrightarrow{0}}{\overrightarrow{0}} \\
\frac{0}{0} \\
2\end{array}$ & $\begin{array}{l}\stackrel{\overrightarrow{0}}{\overrightarrow{0}} \\
\stackrel{0}{2}\end{array}$ & $\stackrel{\stackrel{n}{m}}{\stackrel{m}{m}}$ & $\begin{array}{c}m \\
\dot{m} \\
m\end{array}$ & $\stackrel{\bar{\infty}}{\sim}$ & $\bar{\sim}$ & $\overline{\dot{m}}$ & 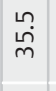 & $\begin{array}{l}\infty \\
\infty \\
\infty \\
\sim\end{array}$ & $\stackrel{\stackrel{\llcorner}{0}}{\stackrel{m}{m}}$ & $\begin{array}{l}\stackrel{\varphi}{\dot{m}} \\
\stackrel{\text { n }}{2}\end{array}$ & 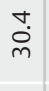 & 衣 & $\stackrel{+}{\dot{m}}$ & $\begin{array}{l}\stackrel{n}{\stackrel{n}{m}} \\
\stackrel{m}{m}\end{array}$ & 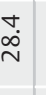 & 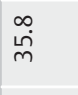 & $\frac{0}{\dot{m}}$ & $\stackrel{\vec{\infty}}{\sim}$ & 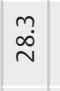 & 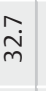 & 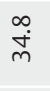 \\
\hline 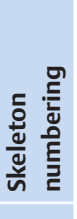 & 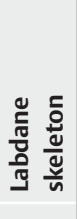 & 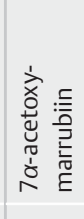 & 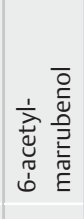 & 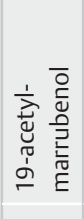 & 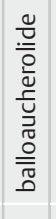 & 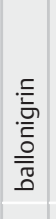 & 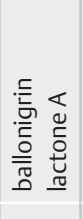 & 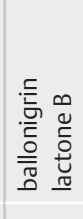 & 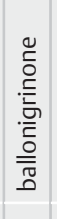 & 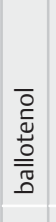 & 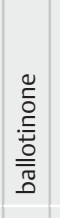 & 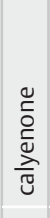 & 竞 & 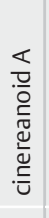 & 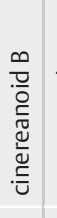 & 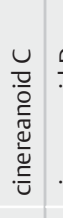 & 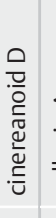 & 这 & 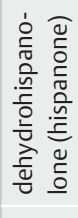 & 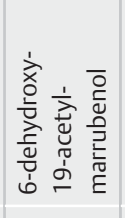 & 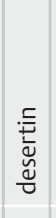 & 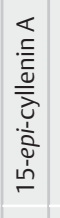 & 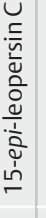 & 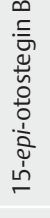 \\
\hline & & - & $N$ & $m$ & $\sigma$ & in & 6 & ^ & $\infty$ & $a$ & 웅 & $=$ & $\simeq$ & $\stackrel{m}{=}$ & $\Xi$ & $\stackrel{\text { n }}{\circ}$ & $\stackrel{\varphi}{\circ}$ & $\approx$ & $\stackrel{\infty}{\sim}$ & $\stackrel{9}{-}$ & ి & $\overline{\boldsymbol{N}}$ & $\mathbf{N}$ & $\tilde{\tilde{N}}$ \\
\hline
\end{tabular}




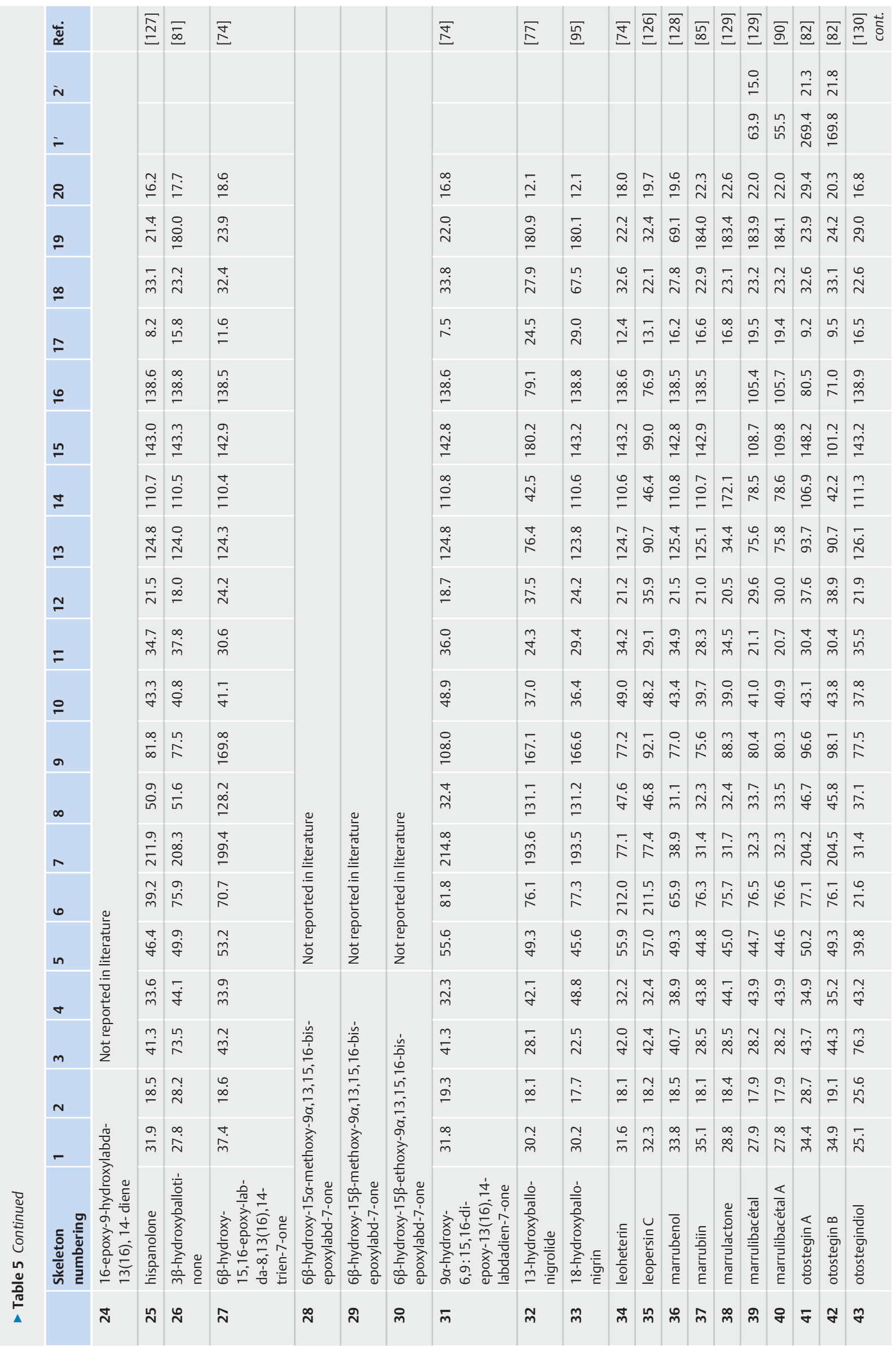




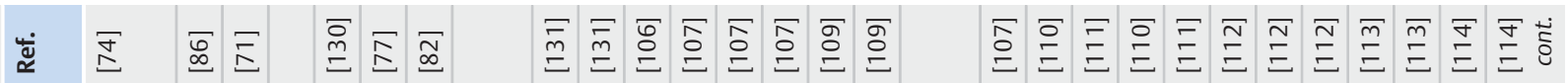

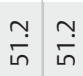

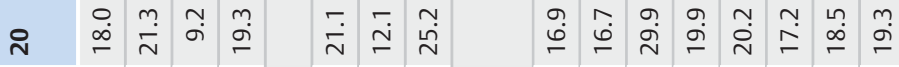

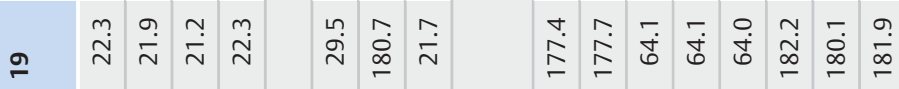

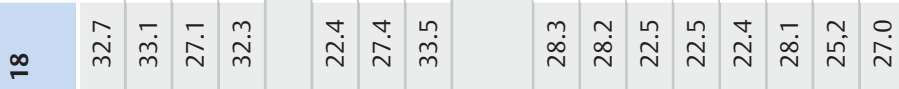

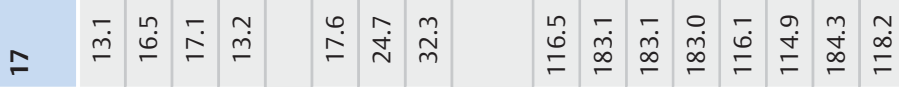

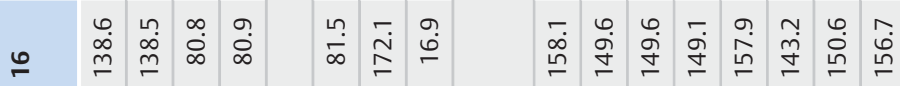

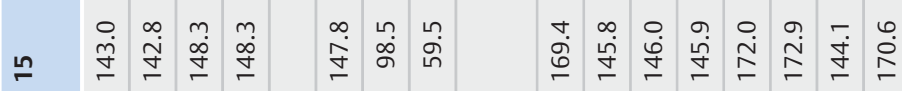

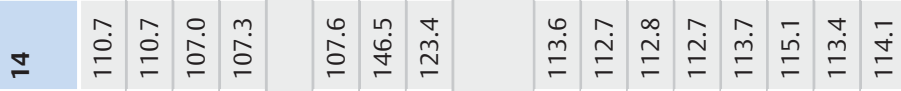

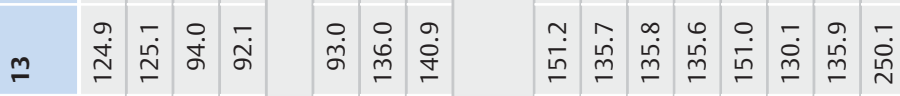

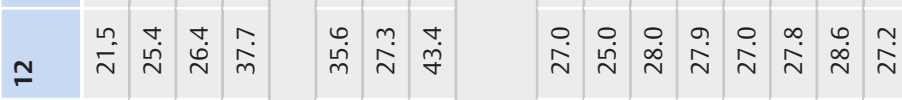

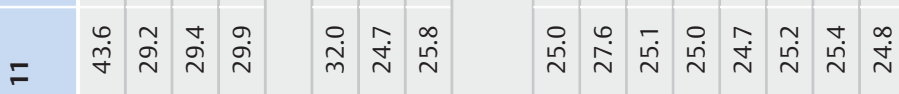

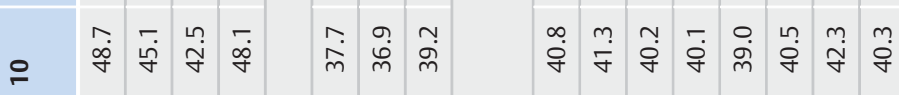

○.

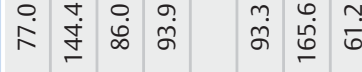

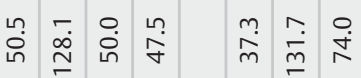

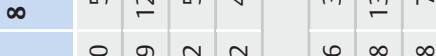

官

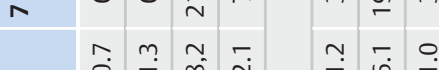

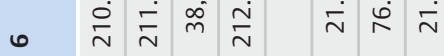

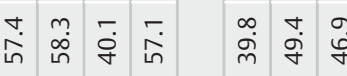

in

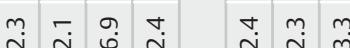

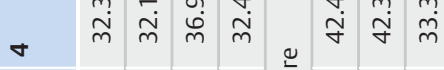

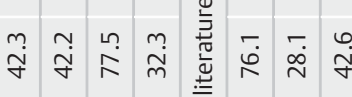

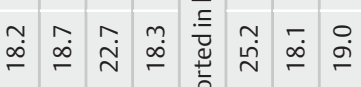

N

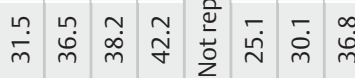

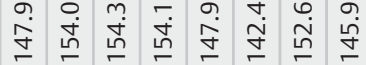

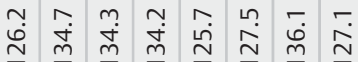

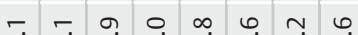

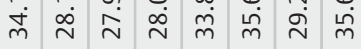

ก.

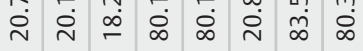

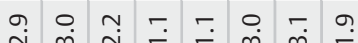

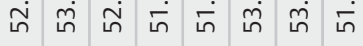

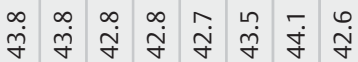

オ. オ.

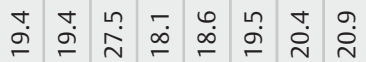

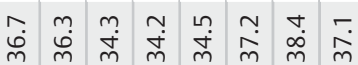

i

ک.

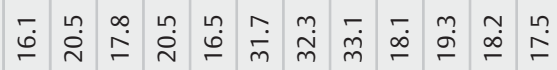

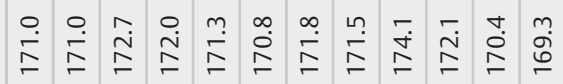

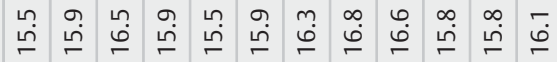

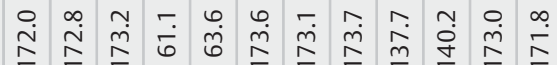

盛

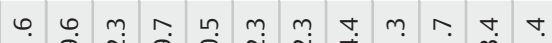

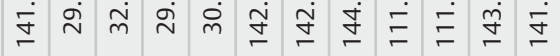

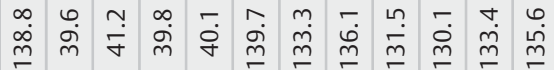

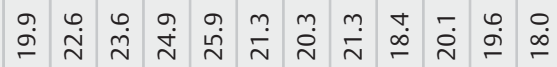

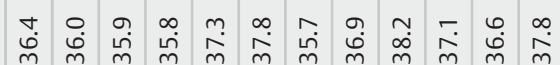

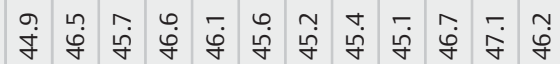

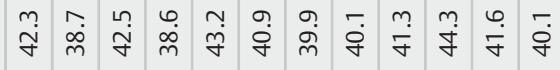

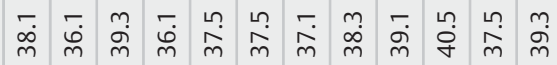

จ.

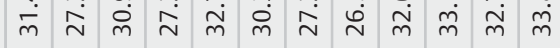

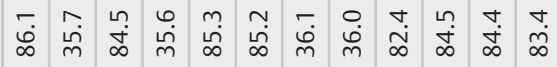

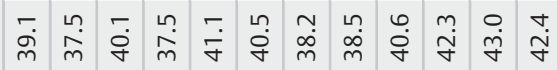

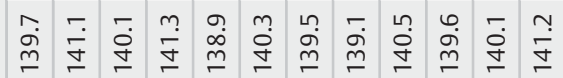

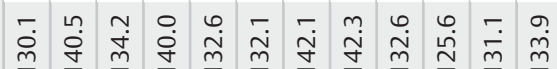

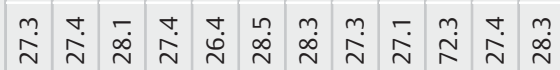

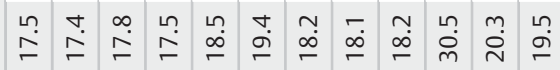




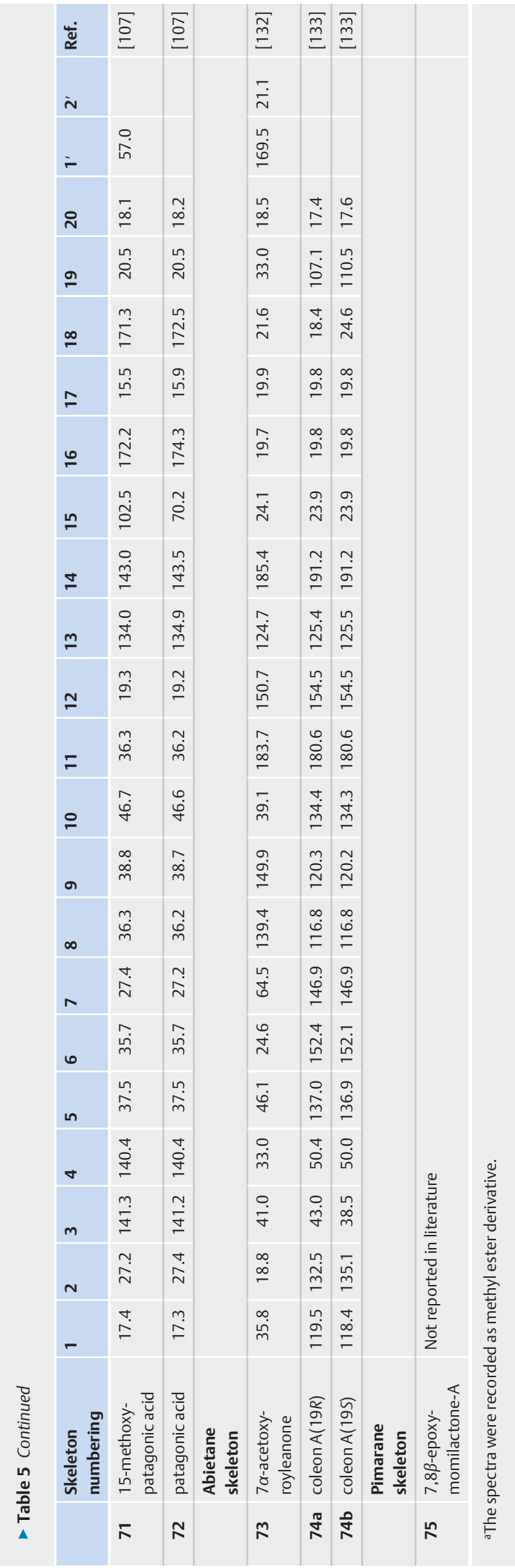

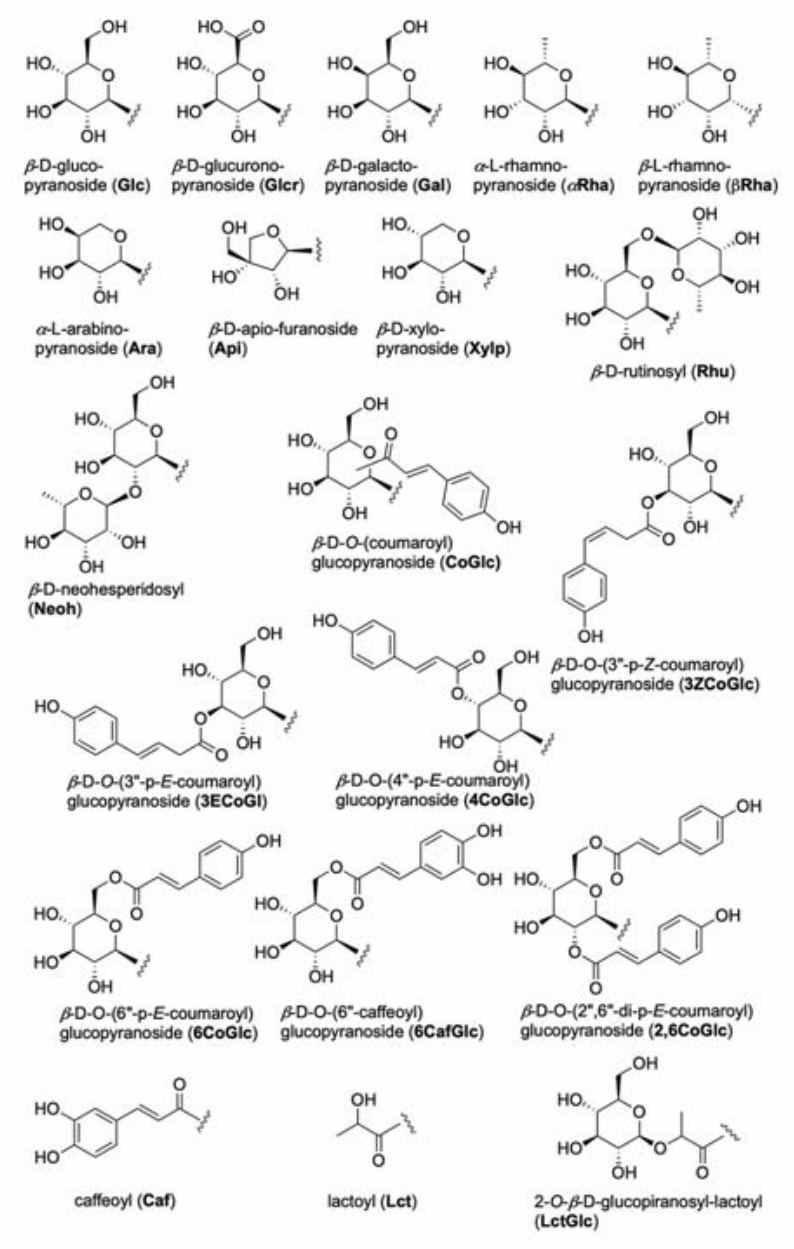

- Fig. 4 Structures of sugars and acyl moieties.

rats with a 7 week treatment of STZ. Afterward, the plasma concentration of malondialdehyde (MDA), co-enzyme $\mathrm{Q}_{9}, \alpha$ - and $\gamma$ tocopherol were measured. A normalization in MDA values was observed in animals treated with $60 \mathrm{mg} / \mathrm{kg}$ b.w. of compound 139, while the $\mathrm{Q}_{9}$ and $y$-tocopherol level remained comparable with those of negative controls.

\section{Anti-inflammatory activity}

This is mainly attributed to the presence of flavonoids and tannins in both genera. The bioactivity is generally evaluated in in vivo models. The aqueous extract of Ballota glandulosissima Hub.Mor. \& Patzak leaves collected in Turkey [245] administrated to rats with carrageenan-induced paw edema $(100 \mathrm{mg} / \mathrm{kg}$ b.w.) was able to induce a significant reduction of the edema volume (32\%). The aerial part aqueous extract of Ballota inaequidens Hub.-Mor. \& Patzak from Turkey [246] showed similar effects by reducing paw edema in rats in a dose-dependent manner: the volume reduction coefficient ranged from 58 to $86 \%$ by administrating 50 to $200 \mathrm{mg} / \mathrm{kg}$ b.w. per day of extract. This species also showed significant, positive dose-dependent results in the abdominal stretching test in mice: $44-91 \%$ reduction by administrating 30 $100 \mathrm{mg} / \mathrm{kg}$ b.w. per day of dry extract. A relevant anti-inflamma- 


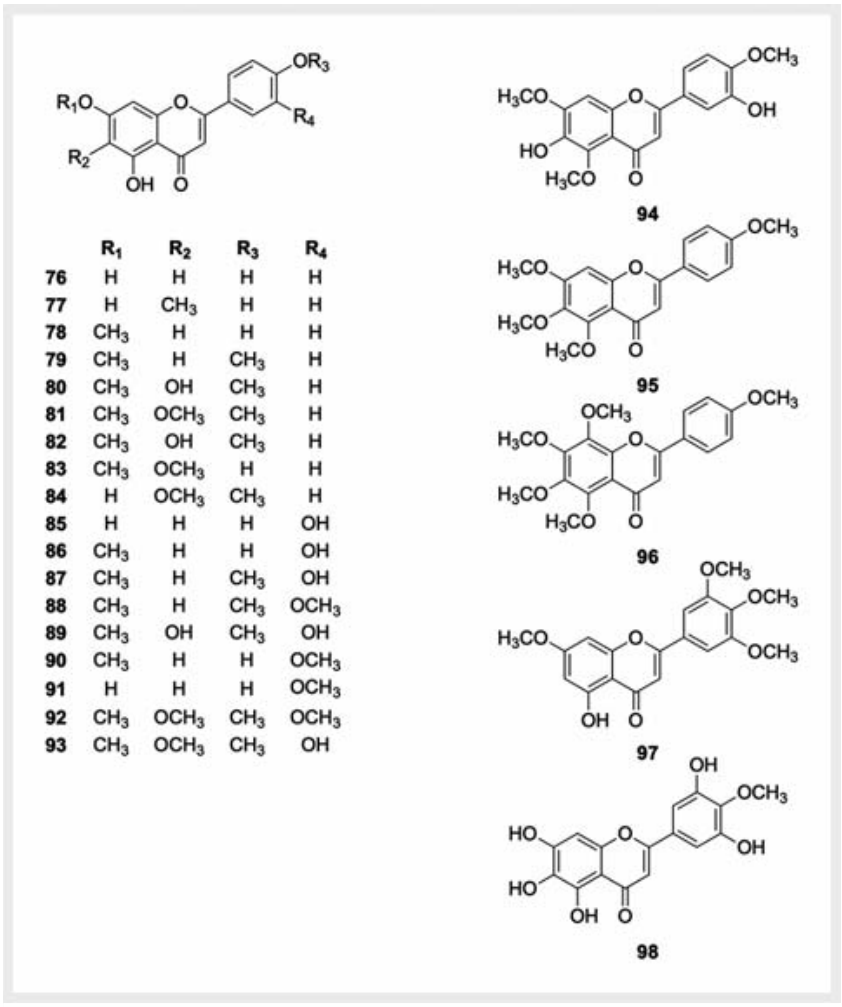

- Fig. 5 Structures of flavones.

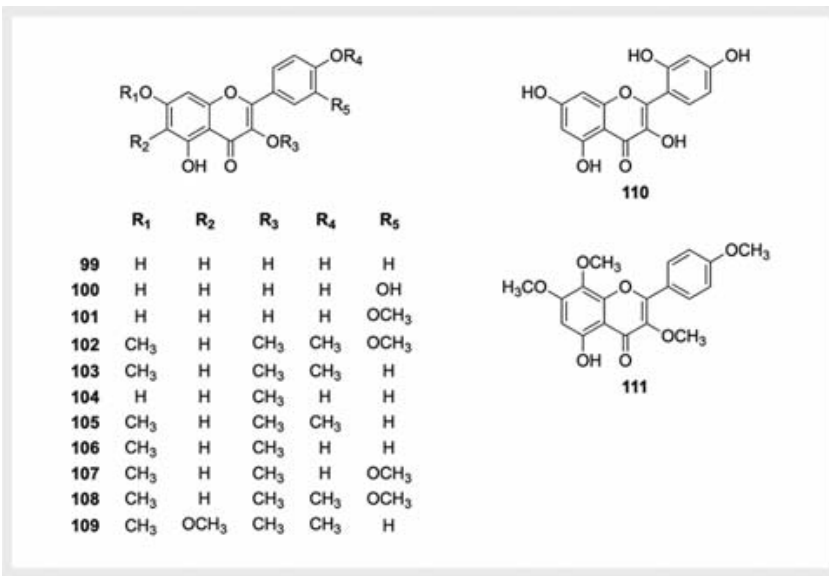

Fig. 6 Structures of flavonols.

tory activity was also demonstrated in the methanolic extract of the AP of B. pseudodictamnus. This species is included in a comparative study on the anti-inflammatory activity of endemic flora from Libya [247]. In the mice paw edema test, a single dose of $500 \mathrm{mg} / \mathrm{kg}$ b. w. reduced the edema volume by $51 \%$.

The same animal model was employed in the evaluation of the anti-inflammatory activity of the crude extract of the aerial part of O. persica [166], as well as the fractions soluble in organic solvents of different polarities ( $\mathrm{PE}, \mathrm{CHCl}_{3}$, EtOAc, $n-\mathrm{BuOH}, \mathrm{MeOH}$ ). The analgesic activities were also investigated. Both the fraction in $\mathrm{BuOH}$ and in $\mathrm{MeOH}$ were able to reduce the edema volume, the last one
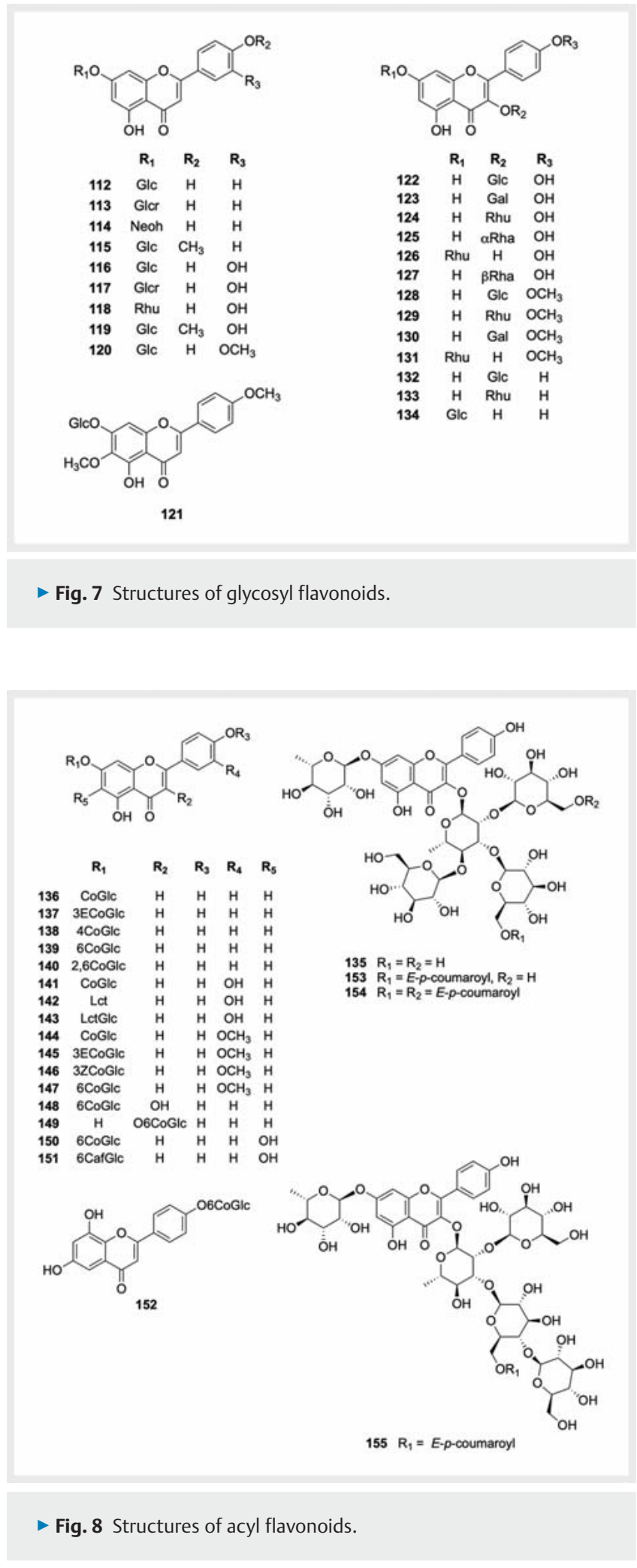

showing an efficacy comparable to that of indomethacin. Furthermore, the $\mathrm{MeOH}$ fraction showed analgesic activity with $\mathrm{EC}_{50}$ of $85.9 \mathrm{mg} / \mathrm{kg}$ b.w. Two active compounds were isolated from the methanolic fraction: vicenin-2 (159) and isorhamnetin-3-O- $\beta$-Dglucopyranoside (128). 

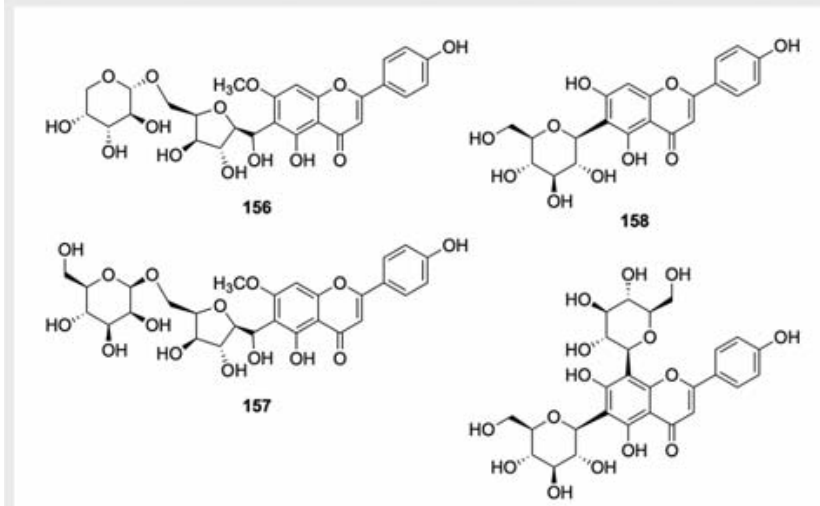

159

Fig. 9 Structures of C-glycosyl flavones.

The extract of $B$. deserti (Algeria) was obtained from AP with PE, dichloromethane, and $\mathrm{MeOH}$. Their anti-inflammatory activity was assessed, once again with the paw edema model, using Wister albino rats [248]. The edema volume was reduced by $85.5 \%$ by a dose of $200 \mathrm{mg} / \mathrm{kg}$ b.w after $3 \mathrm{~h}$ of the $\mathrm{MeOH}$ extract, while acute toxicity signs were absent until $5000 \mathrm{mg} / \mathrm{kg}$ b. w. The lowest, but still significant effect, was obtained in another study [248] with the aqueous extract of the same taxa: edema volume was reduced after $3 \mathrm{~h}$ from 11 to $44 \%$ at variable doses from 250 to $1000 \mathrm{mg} / \mathrm{kg}$ b. w.

The EtOAc extract of the aerial part of B. lanata from China [144] was investigated for its potential anti-inflammatory activity in 2 animal models: the paw edema induced by carrageenan and egg albumin in rats. The effect of edema reduction after treatment with 100 to $400 \mathrm{mg} / \mathrm{kg}$ b. w. of extract was dose-dependent: at the highest dose, there was an effect statistically comparable with the positive reference luteolin (85). Another study reported the isolation of apigenin-7-O- $\beta$-D-(6"'-E-p-coumaroyl)glucopyranoside (139), apigenin-7-O- $\beta$-D-(2",6"-E-dicoumaroyl)glucopyranoside (140), and verbascoside (227) from the ethanolic extract of B. lanata (China) [168]. Their anti-inflammatory activity was investigated in the same animal models employed for the plant extract. Compound 227 had a higher anti-inflammatory potential than diclofenac ( $5 \mathrm{mg} / \mathrm{kg}$ b.w.), while the activity of compounds 139 and 140 were similar at $20-50 \mathrm{mg} / \mathrm{kg}$ b.w. doses. The butanol extract of this species furnished 2 other potentially anti-inflammatory compounds: the flavone C-glycosides panzeroside A (156) and B (157) [173]. A paw edema reduction volume in rats equal to that of $5 \mathrm{mg} / \mathrm{kg}$ b.w. of diclofenac was achieved with $30 \mathrm{mg} / \mathrm{kg}$ b.w. of both compounds.

\section{Antibacterial activity}

Because of the growing human health danger represented by the antibiotics-resistant bacterial strains and in consideration of the therapeutic uses of many species of Ballota and Otostegia genera in microbial related pathologies, as well as in wounds and burns treatments, the antimicrobial activity of EOs, plant parts extracts, and also individual compounds has been extensively investigated.

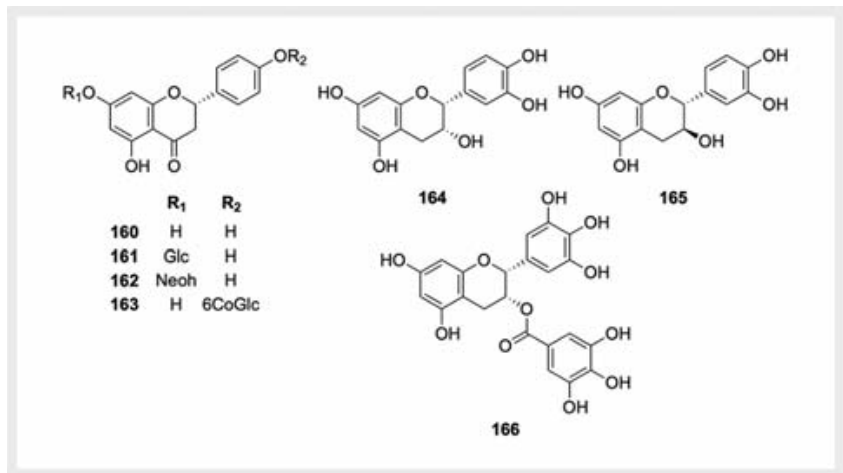

- Fig. 10 Structures of flavanones and flavanols.

- Table 14 reports a synopsis of the most relevant evidence in the literature.

The MIC and MBC values may vary over a wide interval and are often lower in order of magnitude than that of the antibiotics selected as positive controls. For example, the ethanolic extract of $B$. acetabulosa was found to be significantly active against Escherichia coli with MIC and MBC values equal to ampicillin (32, and $64 \mu \mathrm{g} / \mathrm{mL}$, respectively). On the contrary, the same extract was inactive against Pseudomonas aeruginosa (Schroeter) Migula (MIC and MBC $1.02 \mathrm{mg} / \mathrm{mL}$ ) [250].

The direct relation between the chemical composition of the plant extracts and the antimicrobial activity is not always clearly explainable. Sometimes this relation is evident as in the case of the antimicrobial activity of $B$. pseudodictamnus EO from Greece [220]. Its antibiotic activity was assessed against a panel of 6 pathogenic microorganisms, along with the activity of the principal components of the oil individuated by GC-MS. Unsurprisingly, the MIC of the main component caryophillene oxide (from 0.07 to $5.20 \mu \mathrm{g} / \mathrm{mL}$ ) was from 4 to 6 times stronger than that of the whole oil, in which caryophillene oxide is present at $22.8 \%$ ! The author stated, "The antibacterial property of the oil is suspected to be associated with the high percentage of caryophyllene oxide which is known to possess strong antibacterial activity." The data reported seem to show any other component of this EO as a mere diluent! This is just an example of how the rather vague postulate of the "synergistic effect" of natural product mixtures in biological systems should at least be deeply questioned. Another example of antibacterial activity of a caryophillene rich EO is in the study of $B$. saxatilis subsp. brachyodonta from Turkey [221]. A MIC of $50 \mu \mathrm{g} / \mathrm{mL}$ against all of the bacterial genera evaluated was found; caryophillene, caryophillene oxide, and epi-bicyclosesquiphellandrene were the main components of the oil.

An example of the lack of a molecular explanation is the study of the activity of the water and methanolic extract of leaves of B. africana from South Africa [251]: MICs were reported at 438 and $370 \mu \mathrm{g} / \mathrm{mL}$ versus Klebsiella pneumoniae (Schroeter) Trevisan for methanolic and water extracts, respectively. However, an attempt to isolate single bioactive compounds using bio-guided fractionation of the crude extract failed. Additionally, the authors underlined, for not so evident reasons, the apparent discrepancy between the observed antibacterial activity and the lack of 
- Table 6 Distribution of flavones in Ballota and Otostegia taxa.

\begin{tabular}{|c|c|c|}
\hline No & Name & Taxa \\
\hline 76 & apigenin & $\begin{array}{l}\text { B. acetabulosa }[75,134,135] \text {; B. deserti [89]; B. hirsuta }[136,137] \text {; B. lanata [138]; } \\
\text { B. nigra [139]; B. pilosa [140] }\end{array}$ \\
\hline 77 & 6-methylapigenin & O. persica $[141,142]$ \\
\hline 78 & genkwanin & B. hirsuta [136] \\
\hline 79 & apigenin 7,4'-dimethyl ether & B. inaequidens [73, 75, 76]; B. lanata [143-145]; B. pseudodictamnus [79]; B. rotundifolia [73] \\
\hline 80 & ladanein & $\begin{array}{l}\text { B. acetabulosa [73]; B. hirsuta [136]; B. inaequidens [75]; B. latibracteolata [73]; B. nigra [115]; } \\
\text { B. rotundifolia [73]; B. saxatilis [73]; B. saxatilis ssp. brachyodonta [146] }\end{array}$ \\
\hline 81 & salvigenin & B. glandulosissima $[75,147] ;$ B. hirsuta $[136]$ \\
\hline 82 & scutellarein 7,4'-dimethyl ether & B. acetabulosa $[75,134]$ \\
\hline 83 & cirsimaritin & B. andreuzziana [148]; B. pilosa [140] \\
\hline 84 & pectolinarigenin & O. fruticosa [149] \\
\hline 85 & luteolin & B. acetabulosa [135]; B. hirsuta [136]; B. lanata [138]; B. nigra [139] \\
\hline 86 & luteolin 7-methyl ether & B. andreuzziana [148] \\
\hline 87 & pilloin & B. cinerea $[87]$ \\
\hline 88 & luteolin-7,3', $4^{\prime}$-trimethyl ether & B. glandulosissima $[73,75,147]$; B. inaequidens $[73,75,76]$; B. lanata [145] \\
\hline 89 & nuchensin & B. hirsuta [136] \\
\hline 90 & velutin & B. glandulosissima $[73,75,147]$; B. undulata $[150]$ \\
\hline 91 & chrysoeriol & B. lanata [138]; B. nigra [139]; O. persica [141, 142] \\
\hline 92 & 5-hydroxy-3',4',6,7-tetramethoxy flavone & O. limbata [151] \\
\hline 93 & eupatorin & O. limbata $[151,152]$ \\
\hline 94 & 3',6-dihydroxy-4',5,7-trimethoxy-flavone & O. persica [153] \\
\hline 95 & 4',5,6,7-tetramethoxyflavone & B. cinerea $[154]$ \\
\hline 96 & tangeretin & B. nigra [155] \\
\hline 97 & corymbosin & B. glandulosissima $[73,75,147]$ \\
\hline 98 & 6,5'-dihydroxy diosmetin & O. fruticosa [156] \\
\hline
\end{tabular}

- Table 7 Distribution of flavonols in Ballota and Otostegia taxa.

\begin{tabular}{|c|c|c|}
\hline No & Name & Taxa \\
\hline 99 & kaempferol & B. deserti [157]; B. lanata $[29,138,145]$; O. persica $[158]$ \\
\hline 100 & quercetin & B. deserti [157]; B. lanata $[29,138,143]$; B. macrodonta $[159]$; O. persica $[158,160]$ \\
\hline 101 & isorhamnetin & B. lanata $[138,145]$ \\
\hline 102 & quercetin $3,7,3^{\prime}, 4^{\prime}$-tetramethyl ether & B. undulata [150] \\
\hline 103 & 5-hydroxy-3,7,4'-trimethoxyflavone & B. inaequidens [73, 76]; B. nigra ssp. foetida [73]; B. rotundifolia [73]; B. saxatilis $[73,75]$ \\
\hline 104 & isokaempferide & B. hirsuta $[136]$ \\
\hline 105 & kaempferol 3,7,4'-trimethyl ether & B. saxatils ssp. brachyodonta [146]; B. undulata [150] \\
\hline 106 & kumatakenin & $\begin{array}{l}\text { B. glandulosissima }[73,75,147] \text {; B. hirsuta [136]; B. nigra ssp. anatolica [73]; B. nigra ssp. } \\
\text { foetida [73] }\end{array}$ \\
\hline 107 & pachypodol & B. glandulosissima $[73,75,147]$; B. inaequidens $[73,75,147]$; B. undulata $[150]$ \\
\hline 108 & retusin & $\begin{array}{l}\text { B. glandulosissima [147]; B. inaequidens [75, 76]; B. nigra ssp. foetida [73]; B. saxatilis [73]; } \\
\text { B. saxatils ssp. brachyodonta [146] }\end{array}$ \\
\hline 109 & 5-hydroxy-3,6,7,4'-tetramethoxy flavone & B. inaequidens [73, 76]; B. saxatilis [73] \\
\hline 110 & morin & O. persica $[153,160]$ \\
\hline 111 & filindulatin & B. inaequidens [75] \\
\hline
\end{tabular}


- Table 8 Distribution of flavonoid glycosides, flavanones, and flavanols in Ballota and Otostegia taxa.

\begin{tabular}{|c|c|c|}
\hline \multirow[t]{2}{*}{ No } & Name & Taxa \\
\hline & Flavone glycosides & \\
\hline 112 & apigenin-7-O- $\beta$-D-glucopyranoside & $\begin{array}{l}\text { B. acetabulosa [161]; B. deserti [89, 157]; B. hirsuta [136,137]; } \\
\text { B. lanata [138, 144]; B. larendana [162]; B. nigra ssp. foetida [163]; } \\
\text { B. pseudodictamnus [162]; B. undulata [164]; O. persica }[141,142]\end{array}$ \\
\hline 113 & apigenin-7-O- $\beta$-D-glucuronide & B. deserti [89] \\
\hline 114 & apigenin-7-O- $\beta$ - neohesperidoside & B. deserti $[89,90]$ \\
\hline 115 & acacetin-7-O- $\beta$-D-glucopyranoside & B. acetabulosa $[75,134]$ \\
\hline 116 & luteolin-7-O- $\beta$-D-glucopyranoside & $\begin{array}{l}\text { B. andreuzziana }[148] \text {; B. hirsuta }[136,137] \text {; B. lanata }[29,138] \text {; } \\
\text { B. larendana }[162] \text {; B. macrodonta }[159] \text {; B. undulata }[150,164]\end{array}$ \\
\hline 117 & luteolin-7-O- $\beta$-D-glucuronide & O. fruticosa [156] \\
\hline 118 & luteolin-7-O- $\beta$-D-rutinoside & B. hirsuta [136] \\
\hline 119 & diosmetin-7-O- $\beta$-D-glucopyranoside & B. undulata $[150]$ \\
\hline 120 & chrysoeriol-7-O- $\beta$-D-glucopyranoside & $\begin{array}{l}\text { B. acetabulosa [75, 134, 161]; B. hirsuta [137]; B. pseudodictamnus [162]; } \\
\text { O. fruticosa [149] }\end{array}$ \\
\hline \multirow[t]{2}{*}{121} & 6,4'-di-O-methyl-scutellarein-7-O- $\beta$-glucopyranoside & B. andreuzziana [148] \\
\hline & Flavonol glycosides & \\
\hline 122 & quercetin-3-O- $\beta$-D-glucopyranoside (isoquercetin) & B. cinerea [88]; B. hirsuta [136]; B. lanata [138] \\
\hline 123 & quercetin-3-O- $\beta$-galactopyranoside & B. lanata $[144]$ \\
\hline 124 & rutin & $\begin{array}{l}\text { B. acetabulosa [135]; B. cinerea [88]; B. deserti [157]; B. lanata }[29,138 \text {, } \\
\text { 143]; B. macrodonta [159]; B. undulata [164] }\end{array}$ \\
\hline 125 & quercetin-3-O- $\alpha$-L-rhamnopyranoside & B. lanata $[29,138]$ \\
\hline 126 & quercetin-3-O- $\beta$-L-rhamnopyranoside & B. cinerea $[165]$ \\
\hline 127 & quercetin-7-O- $\beta$-L-rutinoside & B. andreuzziana [148] \\
\hline 128 & isorhamnetin-3-O- $\beta$-D-glucopyranoside & B. lanata $[138,144,145]$; O. persica $[166]$ \\
\hline 129 & isorhamnetin-3-O- $\beta$-D-rutinoside & B. lanata $[138,144]$ \\
\hline 130 & isorhamnetin-3-O- $\beta$-D-galactopyranoside & B. lanata [138] \\
\hline 131 & isorhamnetin-7-O- $\beta$-D- rutinoside & B. lanata $[145]$ \\
\hline 132 & kaempferol-3-O- $\beta$-D-glucopyranoside & B. lanata $[138,143,145]$ \\
\hline 133 & kaempferol-3-O- $\beta$-D-rutinoside (nicotiflorin) & B. cinerea [88]; B. lanata [138] \\
\hline 134 & kaempferol-7-O- $\beta$-D-glucopyranoside & B. lanata [138] \\
\hline \multirow[t]{2}{*}{135} & $\begin{array}{l}\text { kaempferol-3-O-[ } \beta \text {-D-glucopyranosyl-( } 1 \rightarrow 2)-\{\beta \text {-D-glucopyranosyl- } \\
(1 \rightarrow 3)\}-\{\beta \text {-D-glucopyranosyl-( }(1 \rightarrow 4)\} \text { - } \alpha \text {-L-rhamnopyranoside]-7-O- } \\
{[\alpha \text {-L-rhamnopyranoside }]}\end{array}$ & O. limbata [167] \\
\hline & Acyl flavonoid glycosides & \\
\hline 136 & apigenin-7-( $p$-coumaroyl)-glucoside & B. hirsuta $[136,137]$ \\
\hline 137 & apigenin-7-O- $\beta$-D-(3"'-p-E-coumaroyl)glucopyranoside & B. larendana [162] \\
\hline 138 & apigenin-7-O- $\beta$-D-(4"'-E-p-coumaroyl)glucopyranoside (echinaticin) & B. acetabulosa [161]; O. persica $[141,142]$ \\
\hline 139 & apigenin-7-O- $\beta$-D-(6"'-E-p-coumaroyl)glucopyranoside (terniflorin) & B. deserti [89]; B. lanata [138, 168, 169]; B. larendana [162]; B. pilosa [140] \\
\hline 140 & apigenin-7-O- $\beta$-D-(2",6"-E-dicoumaroyl)glucopyranoside & B. lanata [168] \\
\hline 141 & luteolin-7-(p-coumaroyl)glucopyranoside & B. hirsuta [137] \\
\hline 142 & luteolin-7-lactate & B. nigra $[170]$ \\
\hline 143 & luteolin-7-O-[2-O- $\beta$-D-glucopiranosyl-lactate] & B. nigra $[170]$ \\
\hline 144 & chrysoeriol-7-(p-coumaroyl)glucopyranoside & B. hirsuta [137] \\
\hline 145 & chrysoeriol-7-O- $\beta$-D-(3"'-E-p-coumaroyl)-glucopyranoside & B. acetabulosa [161]; B. pseudodictamnus [162] \\
\hline 146 & chrysoeriol-7-O- $\beta$-D-(3"'-Z-p-coumaroyl)glucopyranoside & B. acetabulosa [161] \\
\hline 147 & chrysoeriol-7-O- $\beta$-D-(6"'-p-coumaroyl)glucopyranoside & B. lanata [138]; B. undulata [150] \\
\hline 148 & kaempferol-7-O- $\beta$-D-(6"'-p-coumaroyl)glucopyranoside & B. lanata [138] \\
\hline 149 & kaempferol-3-O- $\beta$-D-(6"'-p-coumaroyl)glucopyranoside & B. lanata [138] \\
\hline
\end{tabular}


- Table 8 Continued

\begin{tabular}{|c|c|c|}
\hline No & Name & Taxa \\
\hline 150 & $\begin{array}{l}\text { 5,6,7,4'-tetrahydroxyflavone-7-O- } \beta \text {-D- } \\
\left(6^{\prime \prime}-E-p \text {-coumaroyl)glucopyranoside }\right.\end{array}$ & B. lanata [144] \\
\hline 151 & $\begin{array}{l}\text { 5,6,7,4'-tetrahydroxyflavone-7-O- } \beta \text {-D- } \\
\left(6^{\prime \prime} \text {-E-caffeoyl)glucopyranoside }\right.\end{array}$ & B. lanata [144] \\
\hline 152 & leufolins B & B. arabica $[171]$ \\
\hline 153 & $\begin{array}{l}\text { 5-hydroxy-2-(4-hydroxyphenyl)-4-oxo-7-[( } \alpha \text {-L-rhamnopyranosyl)oxy]- } \\
4 \text { H-chromen-3-yl } \beta \text {-D-glucopyranosyl-( } 1 \rightarrow 2) \text {-[ } \beta \text {-D-glucopyranosyl- } \\
(1 \rightarrow 4)]-[6-O-[(2 E)-3 \text {-(4-hydroxyphenyl) prop-2-enoyl]- } \beta \text {-D-gluco- } \\
\text { pyranosyl-( }(\rightarrow 3)]-\alpha \text {-L-rhamnopyranoside }\end{array}$ & O. limbata [172] \\
\hline 154 & $\begin{array}{l}\text { 5-hydroxy-2-(4-hydroxyphenyl)-4-oxo-7-[( } \alpha \text {-L-rhamnopyranosyl)oxy]- } \\
4 \text { H-chromen-3-yl [6-O-[(2E)-3-(4-hydroxyphenyl)prop-2-enoyl]- } \beta \text {-D- } \\
\text { glucopyranosyl-( }(1 \rightarrow 2)] \text {-[ } \beta \text {-D-glucopyranosyl-( } 1 \rightarrow 4)] \text {-[6-O-[(2E)-3- } \\
\text { (4-hydroxyphenyl)prop-2-enoyl]- } \beta \text {-D-glucopyranosyl-( }(\rightarrow 3)]-\alpha-L- \\
\text { rhamnopyranoside }\end{array}$ & O. limbata [172] \\
\hline \multirow[t]{2}{*}{155} & 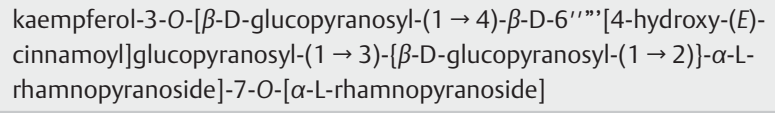 & O. limbata [167] \\
\hline & C-glycosyl flavonoids & \\
\hline 156 & panzeroside $\mathrm{A}$ & B. lanata [173] \\
\hline 157 & panzeroside B & B. lanata [173] \\
\hline 158 & isovitexin & O. persica $[158]$ \\
\hline \multirow[t]{2}{*}{159} & vicenin-2 & $\begin{array}{l}\text { B. aucheri [174]; B. hirsuta [136]; B. nigra ssp. foetida [163]; } \\
\text { O. fruticosa [156]; O. persica [166] }\end{array}$ \\
\hline & Flavanones, flavanone glycosides & \\
\hline 160 & naringenin & B. acetabulosa [135] \\
\hline 161 & naringenin-7-O- $\beta$-D-glucopyranoside & B. macrodonta [159] \\
\hline 162 & naringin & B. acetabulosa [135] \\
\hline \multirow[t]{2}{*}{163} & leufolins A & B. arabica $[171]$ \\
\hline & Flavanols & \\
\hline 164 & epicatechin & B. acetabulosa [135]; B. macrodonta [159] \\
\hline 165 & catechin & B. macrodonta [159] \\
\hline 166 & epigallocatechin gallate & B. macrodonta [159] \\
\hline
\end{tabular}

resveratrol in the extract. Furthermore, in one case, the demonstration of the lack of antibacterial activity versus Propionicbacterium acnes (Gilchrist) Douglas of Italian B. nigra extract demonstrated the inconsistency of its claimed ethnopharmacological use as an anti-acne remedy [262]. In some cases, the antimicrobial activity of a certain species may vary greatly depending on the extraction solvent and the target investigated. A clear example is the case of the aerial part of Iranian O. persica [261]: the initial ethanolic extract was portioned in fractions soluble in $n-\mathrm{Hex}$, $\mathrm{CHCl}_{3}$, and finally $\mathrm{MeOH}$. The lowest MIC and $\mathrm{MBC}$ values were $1.25 \mathrm{mg} / \mathrm{mL}$ for the $\mathrm{CHCl}_{3}$ ext. against Staphylococcus aureus and Enterococcus faecalis, while the $\mathrm{MeOH}$ extc. had a $25 \mathrm{mg} / \mathrm{mL} \mathrm{MIC}$ for Listeria monocytogens (E. Murray et al.) Pirie. Finally, the bacteria species E. coli, P. aeruginosa, Salmonella spp., Klebsiella spp., and Proteus spp. were completely insensitive to all of the extracts.

In other cases, the different geographical origin of the plant material may be the cause of strong differences in the bioactivity, as is the case of the EO derived from $O$. fruticosa. The plant har- vested in Egypt is characterized by antibacterial activity with MIC in the order of $\mu \mathrm{g} / \mathrm{mL}$ [224]. On the contrary, the same taxa from Yemen show MIC values 2 order of magnitude higher than the former [222]. This difference can be explained by the deep difference in the composition of the 2 oils, regarding their main components in particular, as discussed above.

- Table 14 includes cases where the crude extracts were further portioned with organic solvents in the attempt to concentrate the more active compounds [253, 254,257], as well as other cases, where the bioactivity was evaluated for single isolated molecules $[76,89,187]$. In most, highly polar extracts obtained with protic solvents and water contained mainly phenols, phenylpropanoids, and glycosylates. Only 1 case reports the isolation and the measurement of the antibacterial activity of terpenes from the Ac extract of $B$. saxatilis subsp. saxatilis from Turkey [80]. 


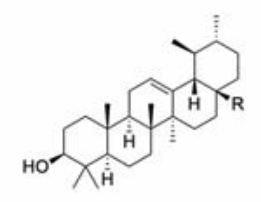

$167 \mathrm{R}=\mathrm{CH}_{3}$

$176 \mathrm{R}=\mathrm{COOH}$

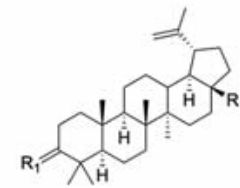

$169 \mathrm{R}=\mathrm{CH}_{2} \mathrm{OH} \mathrm{R}_{1}=\mathrm{H}, \beta-\mathrm{OH}$

$170 \mathrm{R}=\mathrm{COOH} \quad \mathrm{R}_{1}=\mathrm{O}$

$172 \quad \mathrm{R}=\mathrm{CH}_{3} \quad \mathrm{R}_{1}=\mathrm{H}, \mathrm{B}-\mathrm{OH}$

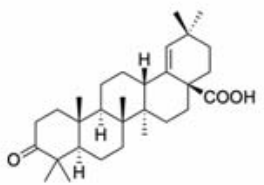

173

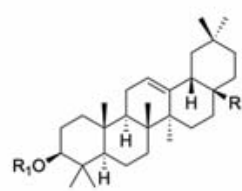

$168 \quad \mathrm{R}=\mathrm{CH}_{3} \quad \mathrm{R}_{1}=\mathrm{H}$

$174 \mathrm{R}=\mathrm{COOH} \mathrm{R}_{1}=\mathrm{H}$

$175 \mathrm{R}=\mathrm{COOH} \mathrm{R}_{1}=\mathrm{AC}$

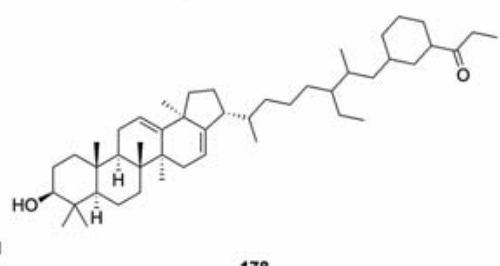

178

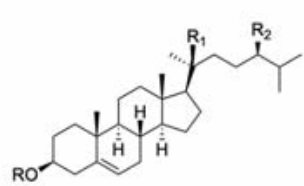

$177 \quad \mathrm{R}=\mathrm{H} \quad \mathrm{R}_{1}=\mathrm{H} \quad \mathrm{R}_{2}=\mathrm{H}$

$179 \mathrm{R}=\mathrm{H} \quad \mathrm{R}_{1}=\mathrm{OH} \Delta_{22} \quad \mathrm{R}_{2}=\mathrm{CH}_{3}$

$180 \mathrm{R}=\mathrm{H} \quad \mathrm{R}_{1}=\mathrm{H} \quad \mathrm{R}_{2}=\mathrm{CH}_{3}$

$181 \mathrm{R}=\mathrm{AC} \quad \mathrm{R}_{1}=\mathrm{H} \quad \mathrm{R}_{2}=\mathrm{CH}_{3}$

$182 \mathrm{R}=\mathrm{Glc} \mathrm{R}_{1}=\mathrm{H} \quad \mathrm{R}_{2}=\mathrm{CH}_{3}$

$183 \quad \mathrm{R}=\mathrm{H} \quad \mathrm{R}_{1}=\mathrm{H} \Delta_{22} \quad \mathrm{R}_{2}=\mathrm{CH}_{3}$

$184 \mathrm{R}=\mathrm{Glc} \quad \mathrm{R}_{1}=\mathrm{H} \Delta_{22} \quad \mathrm{R}_{2}=\mathrm{CH}_{3}$

Fig. 11 Structures of triterpenes and steroids.

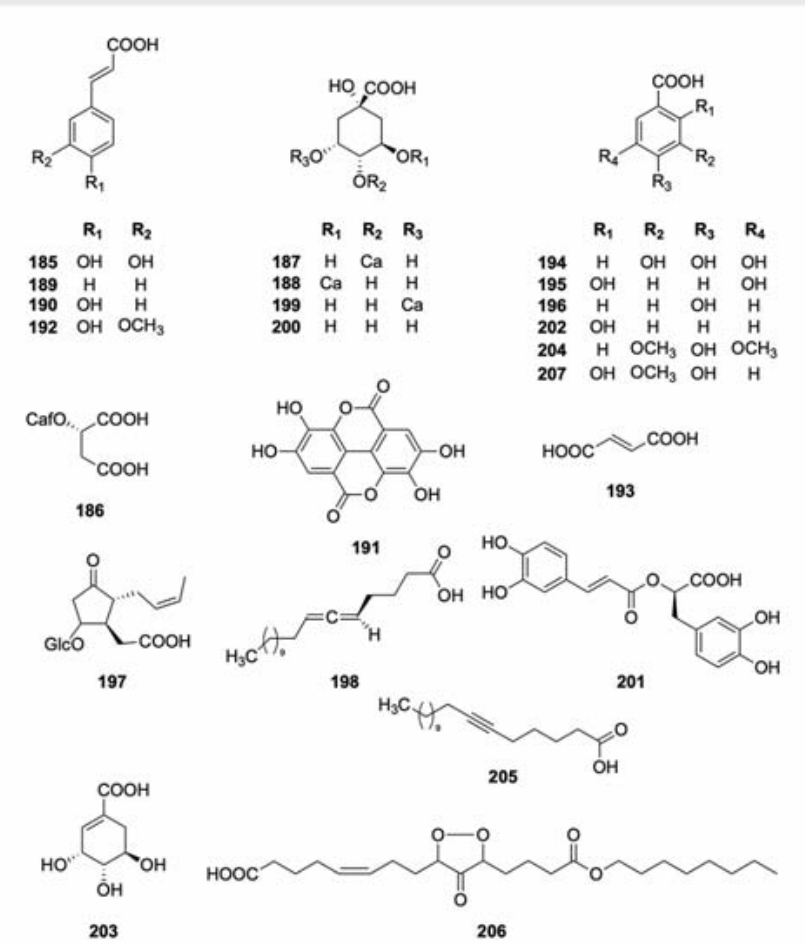

- Fig. 12 Structures of carboxylic acids.
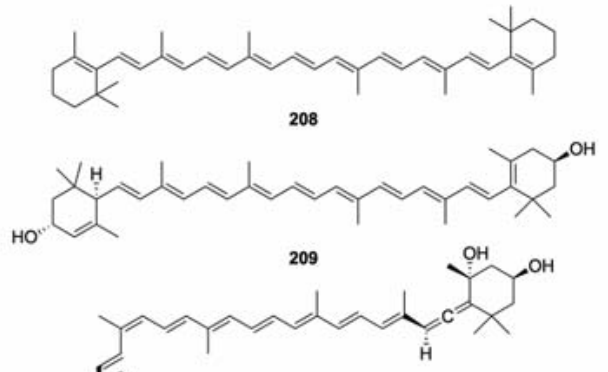

210

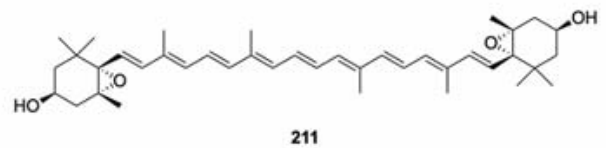<smiles>CC1=C(/C=C/C=C(C)/C=C/C=C/C(C)=C/C=C/C(C)=C/C=C/C=C(C)/C=C/C2=C(C)CC(O)CC2(C)C)C(C)(C)CC(O)C1</smiles>

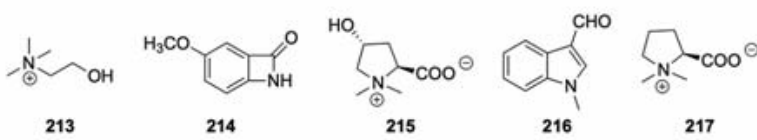

Fig. 13 Structures of carotenoids and nitrogen containing compounds.

\section{Antifungal activity}

The general points discussed for antibacterial activity are valid also in this case, as methodological approaches are obviously similar and a lot of investigations involve both bacterial and fungal species as targets. - Table $\mathbf{1 5}$ summarizes the most relevant data reviewed.

\section{Antitumor activity}

The EO of Ballota undulate, B. saxatilis, and B. nigra collected in Italy were assessed for their in vitro cytotoxicity toward the Hep-G2 hepatocarcinoma and MCF-7 breast carcinoma cell lines [218]. The 3 oils showed moderate inhibition values against the former target (IC $50: 54.7,65.4$, and $69.9 \mu \mathrm{g} / \mathrm{mL}$, respectively) and low values for latter $(>100 \mu \mathrm{M})$. Sesquiterpenes were found as major components in the oils. The MCF-7 cells were instead more sensitive to the EO of $O$. fruticosa with an $\mathrm{IC}_{50}$ of $55.1 \mu \mathrm{g} / \mathrm{mL}$. This taxon was moderately active also against MDA-MB-231 cells with $\mathrm{IC}_{50}$ of $72.3 \mu \mathrm{g} / \mathrm{mL}$ [222].

Rhabdomyosarcoma cells were found to be sensitive to treatment with the methanolic extract of 0 . limbata from Pakistan [266]. Cell viability tests showed up to $93 \%$ mortality after $72 \mathrm{~h}$, a higher level than cisplatin (23\%).

The EtOH extract of AP of B. cinerea was assessed for its cytotoxicity against several cancer cell lines, showing moderate activity [267] with the following LC $_{50}$ values $(\mu \mathrm{g} / \mathrm{mL}): 131.8$ for SK-MEL 2, 275.4 for BE (2) C, and 302.0 for U87MG. 
- Table 9 Distribution of triterpenes, steroids, carboxylic acids, and carotenoids in Ballota and Otostegia taxa.

\begin{tabular}{|c|c|c|}
\hline \multirow[t]{2}{*}{ No } & Names & Taxa \\
\hline & Triterpenoids & \\
\hline 167 & $\alpha$-amyrin & O. fruticosa [175] \\
\hline 168 & $\beta$-amyrin & B. cinerea $[154,176]$, O. persica $[177]$ \\
\hline 169 & betulin & B. cinerea [176], \\
\hline 170 & betulonic acid & B. cinerea $[154]$ \\
\hline 171 & friedelin & B. aucheri [174], B. cinerea [154] \\
\hline 172 & lupeol & O. fruticosa [175] \\
\hline 173 & moronic acid & B. cinerea $[178]$ \\
\hline 174 & oleaonolic acid & B. nigra [179], B. cinerea [180] O. fruticosa [156] \\
\hline 175 & oleanolic acid 3-acetate & B. pilosa $[140]$ \\
\hline \multirow[t]{2}{*}{176} & ursolic acid & B. arabica [181], B. nigra [179], O. fruticosa [156] \\
\hline & Steroids & \\
\hline 177 & campesterol & O. persica [177] \\
\hline 178 & $\begin{array}{l}\text { 3ß-hydroxy-35-(cyclohexyl-5'-propan-7'-one)- } \\
\text { 33-ethyl-34-methyl-bacteriohop-16-ene }\end{array}$ & B. cinerea $[182]$ \\
\hline 179 & leucisterol & B. arabica $[181]$ \\
\hline 180 & $\beta$-sitosterol & $\begin{array}{l}\text { B. arabica }[181], \text { B. cinerea }[87,154,176], \text { B. deserti }[24] \text {, B. lanata }[29,143] \text {, B. nigra }[115,183] \text {, } \\
\text { B. pilosa }[140] \text {, O. fruticosa }[156,175], \text { O. persica }[177]\end{array}$ \\
\hline 181 & $\beta$-sitosterol 3-acetate & O. persica [177] \\
\hline 182 & $\beta$-sitosterol-3-O- $\beta$-D-glucopiranoside & B. cinerea [154], B. deserti [24], B. lanata [29, 143], B. pilosa [140] \\
\hline 183 & stigmasterol & $\begin{array}{l}\text { B. aucheri [174], B. cinerea [87, 176, 182], B. deserti [24], B. lanata [143], B. pilosa [140], } \\
\text { B. undulata [81], O. integrifolia [100], O. persica [177] }\end{array}$ \\
\hline \multirow[t]{2}{*}{184} & stigmasterol-3-O- $\beta$-D-glucopiranoside & B. pilosa $[140]$ \\
\hline & Carboxylic acids & \\
\hline 185 & caffeic acid & $\begin{array}{l}\text { B. acetabulosa [135], B. arabica [184], B. lanata }[29,145], \text { B. macrodonta [159], B. nigra }[139,185] \text {, } \\
\text { O. fruticosa [156], O. persica [177] }\end{array}$ \\
\hline 186 & E-caffeoyl-L-malic acid & B. hirsuta [186], B. lanata [29], B. nigra [185-189], B. pseudodictamnus [162], B. rupestris [186] \\
\hline 187 & 4-O-caffeoylquinic acid & B. macrodonta [159] \\
\hline 188 & chlorogenic acid & B. acetabulosa [135], B. lanata [29], B. macrodonta [159], B. nigra [139, 185] \\
\hline 189 & E-cinnamic acid & B. deserti [157], O. persica [158] \\
\hline 190 & E-coumaric acid & B. acetabulosa [135], B. hirsuta [137], B. macrodonta [159] \\
\hline 191 & ellagic acid & B. macrodonta [159] \\
\hline 192 & ferulic acid & B. arabica [184], B. macrodonta [159], B. nigra [139], O. fruticosa [149] \\
\hline 193 & fumaric acid & B. nigra [185] \\
\hline 194 & gallic acid & B. acetabulosa [135], B. arabica [184], B. cinerea [176], B. deserti [157], B. macrodonta [159] \\
\hline 195 & gentisic acid & B. macrodonta [159] \\
\hline 196 & 4-hydroxy benzoic acid & B. arabica [181], B. deserti [157], B. macrodonta [159], O. persica [177] \\
\hline 197 & jasmonic acid 5'- $\beta$-D-glucopyranosyloxy & B. cinerea $[88]$ \\
\hline 198 & laballenic acid & B. nigra $[188]$ \\
\hline 199 & neochlorogenic acid & B. lanata [29], B. macrodonta [159] \\
\hline 200 & quinic acid & B. nigra [185] \\
\hline 201 & rosmarinic acid & B. acetabulosa [135], B. macrodonta [159] \\
\hline 202 & salicylic acid & B. macrodonta [159] \\
\hline 203 & shikimic acid & B. nigra [185] \\
\hline 204 & syringic acid & B. macrodonta [159] \\
\hline 205 & tariric acid & B. cristata $[190]$ \\
\hline 206 & urticic acid & B. arabica [181] \\
\hline 207 & vanillic acid & B. macrodonta [159] \\
\hline
\end{tabular}


- Table 9 Continued

\begin{tabular}{|l|l|l|}
\hline No & Names & Taxa \\
\hline & Carotenoids & \\
\hline $\mathbf{2 0 8}$ & carotene & B. lanata [29] \\
\hline $\mathbf{2 0 9}$ & lutein & B. lanata [29] \\
\hline $\mathbf{2 1 0}$ & neoxanthin & B. lanata [29] \\
\hline $\mathbf{2 1 1}$ & violaxanthin & B. lanata [29] \\
\hline $\mathbf{2 1 2}$ & zeaxanthin & B. lanata [29] \\
\hline
\end{tabular}

Precalyone (45) was isolated, together with other diterpenes, from the ethanolic extract of the leaves and the stems of $B$. cinerea [86]. This compound was active against the murine P-388 lymphocytic leukemia.

Docking studies of 18 phyto compounds from the plant $B$. nigra of the family Lamiaceae were carried out. From the results, ballotinone (10), and ballonigrin (5) were found to have the best binding efficiency with the active site residues of the protein. The binding energies of the 2 compounds were $-12.0691 \mathrm{kcal} / \mathrm{mol}$ and $-10.2564 \mathrm{kcal} / \mathrm{mol}$, respectively. This study provides a promising anti-cervical cancer inhibitor for further drug development [268].

7 $\alpha$-Acetoxyroyleanone (73), also occurring in B. nigra [115], was shown to be an active anticancer agent against both MIAPaCa-2 and melanoma (MV-3) cancer cell lines $\left(\mathrm{IC}_{50}=4.7\right.$ and $7.4 \mathrm{\mu g} / \mathrm{mL}$, respectively) [269]. Additionally, it also exhibited cytotoxic activity against 5 more human cancer cell lines including, breast (MCF-7), human leukemia (CEM and HL-60), murine skin (B16), and colon cancer (HCT-8) cell lines in the range of $\mathrm{IC}_{50}=0.9-7.6 \mu \mathrm{g} / \mathrm{mL}$. Its cytotoxic activity seemed to be related to inhibition of DNA synthesis [270].

The anticancer activity of marrubenol (36), present in B. pseudodictamnus [79], against osteosarcoma cells along with evaluating its effects on autophagic cell death, reactive oxygen species generation and cell migration and invasion tendency was evaluated. The results indicated that compound 36 exhibited an $I C_{50}$ value of $45 \mu \mathrm{M}$ and exerted its cytotoxic effects in a dose-dependent manner. Moreover, it was observed that the drug inhibited colony formation and induced autophagy dose-dependent [271].

A moderate antiproliferative effect on lung adenocarcinoma cell line (H1975 and XLA-07) and mouse mononuclear macrophage leukemia cell line (RAW264.7) was detected for leoheterin (34) [272], a labdane diterpenoid occurring in B. aucheri [71, 74, 97] and O. fruticosa $[82,98]$.

\section{Other bioactivities in vitro}

The efficacy of $B$. nigra in its traditional neurosedative use was proven by assessing the binding activity in dopaminergic, benzodiazepine, and morphinic receptors of a number of compounds isolated from the leaf extract in $\mathrm{EtOH} / \mathrm{H}_{2} \mathrm{O} 1: 1$ [236]: (+)-(E)-caffeoyl-L-malic acid (186), verbascoside (227), forsythoside B (223), arenarioside (220), and ballotetroside (221). These molecules were active with $\mathrm{IC}_{50}$ values ranging from 0.4 to $10 \mu \mathrm{M}$.

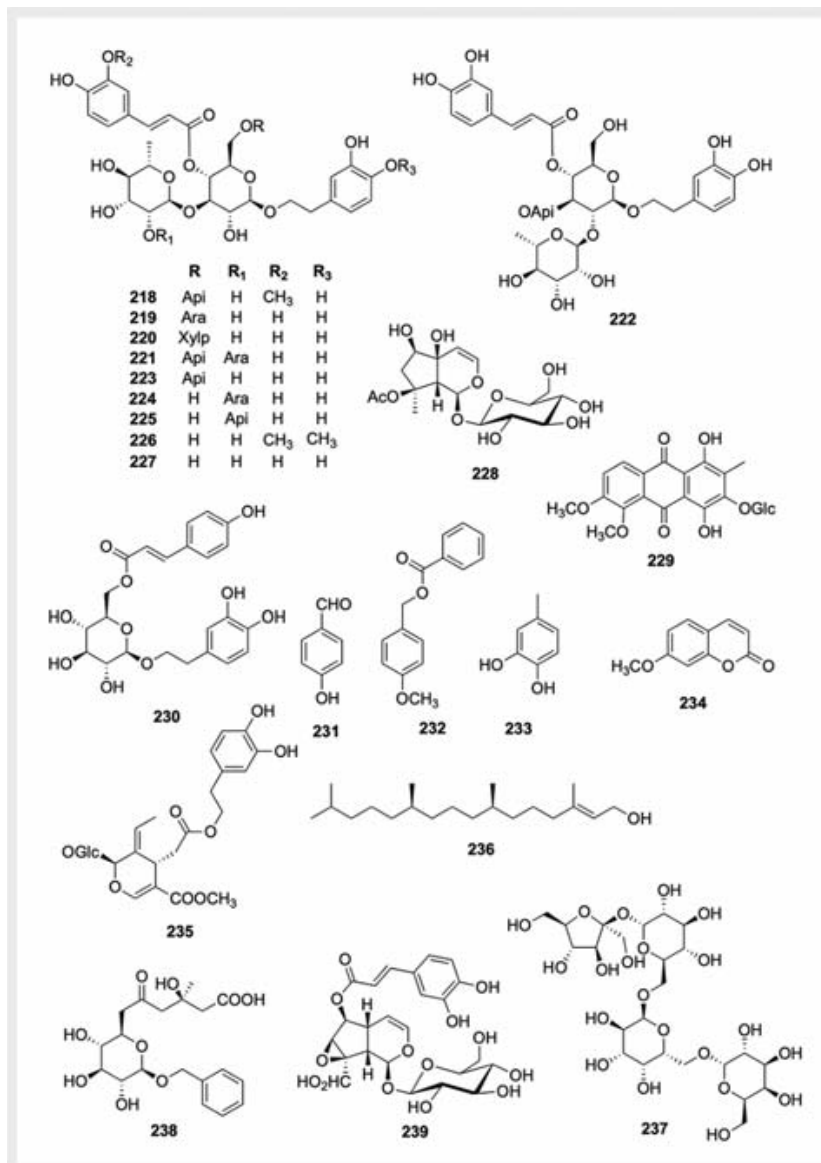

- Fig. 14 Structures of other compounds.

The labdane diterpene cinereanoid D (16), the flavonoid glycosides isoquercetin (122), nicotiflorin (133), and martynoside (226) isolated from the aerial part extract $(95 \% \mathrm{EtOH})$ of $B$. cinerea, collected in India [88], significantly inhibited the ATP binding of a tumor growth-promoting heat shock protein, Hsp90. No significant binding inhibition was revealed for the protein Hsp70.

B. nigra [253] and B. pseudodictamnus [257] from Pakistan were evaluated by the same group for their anti-leishmanial activity. The extracts of the plant stems, leaves, and roots in EtOH were partitioned between water and several organic solvents: $n$-Hex, EtOAc, chloroform, and $n-\mathrm{BuOH}$. The single subfractions of the 
- Table 10 Distribution of $\mathrm{N}$-derivatives, phenylpropanoids, and other metabolites in Ballota and Otostegia taxa.

\begin{tabular}{|c|c|c|}
\hline \multirow[t]{2}{*}{ No } & Names & Taxa \\
\hline & Nitrogen-containing compounds & \\
\hline 213 & choline & B. nigra subsp. foetida [191] \\
\hline 214 & cinerealactam $\mathrm{E}$ & B. cinerea $[88,182]$ \\
\hline 215 & 4-hydroxyprolinebetaine & B. nigra [188], B. undulata [150] \\
\hline 216 & 1-methylindole-3-carboxaldehyde & B. cinerea [87] \\
\hline \multirow[t]{2}{*}{217} & stachydrine & B. lanata $[138,192]$, B. nigra [188], B. nigra subsp. foetida [191], B. undulata [150] \\
\hline & Penylpropanoids & \\
\hline 218 & alyssonoside & B. nigra $[185,187]$ \\
\hline 219 & angoroside $\mathrm{A}$ & B. nigra [187] \\
\hline 220 & arenarioside & B. nigra $[94,187,193]$ \\
\hline 221 & ballotetroside & B. nigra $[94,185,187,194]$ \\
\hline 222 & betonyoside F & B. undulata $[150]$ \\
\hline 223 & forsythoside B & $\begin{array}{l}\text { B. deserti }[89,90], \text { B. hirsuta }[186], \text { B. nigra }[94,115,185-189,193] \text {, } \\
\text { B. pseudodictamnus [162], B. rupestris [186], B. undulata }[150]\end{array}$ \\
\hline 224 & lavandulifolioside & B. nigra [187] \\
\hline 225 & lysionotoside & B. undulata [150] \\
\hline 226 & martynoside & B. cinerea [88], B. nigra [115] \\
\hline \multirow[t]{2}{*}{227} & verbascoside (acteoside) & $\begin{array}{l}\text { B. deserti }[89,90] \text {, B. hirsuta [186], B. lanata }[29,144,168] \text {, B. nigra }[94,185-189,193 \text {, } \\
\text { 195], B. pseudodictamnus [162], B. rupestris [186], B. undulata [150] }\end{array}$ \\
\hline & Other metabolites & \\
\hline 228 & 8-O-acetylharpagide & O. fruticosa [82] \\
\hline 229 & $\begin{array}{l}\text { anthroquinone1,4-dihydroxy-6,7-dimethoxy } \\
\text { 2-methyl 3-O- } \beta \text {-D-glucopyranoside }\end{array}$ & B. cinerea [165] \\
\hline 230 & eutigoside $A$ & B. acetabulosa [161] \\
\hline 231 & 4-hydroxybenzaldehyde & B. macrodonta [159] \\
\hline 232 & 4-methoxybenzyl benzoate & B. arabica [184] \\
\hline 233 & 4-methyl-catechol & B. deserti [157] \\
\hline 234 & 7-methoxy coumarin & B. lanata [145] \\
\hline 235 & oleuropein & B. acetabulosa [135] \\
\hline 236 & phytol & B. deserti [24], B. nigra [183], B. nigra subsp. anatolica [196] \\
\hline 237 & stachyose & B. nigra subsp. foetida [197] \\
\hline 238 & undatuside A & B. cinerea $[88]$ \\
\hline 239 & verminoside & B. undulata $[150]$ \\
\hline
\end{tabular}

2 species showed the ability to inhibit the parasite development process at various stages.

The leaves of $B$. deserti harvested in Tunisia were extracted in a solvent of increasing polarity [25]. $\mathrm{MeOH}, \mathrm{BuOH}$, and EtOAc extracts showed significant antiviral activity against coxsakie B3 virus with $\mathrm{IC}_{50}$ values ranging from 100 to $135 \mu \mathrm{g} / \mathrm{mL}$ and with a selective index above 3 .

The genotoxic and antigenotoxic activities of some $B$. deserti AP extracts in various solvents were evaluated on $E$. coli PQ37 cells by the SOS Chromotest. Additionally, a number of pure compounds isolated from the same plant were included in this investigation [89]. EtOAc, $\mathrm{MeOH}$, and $\mathrm{BuOH}$ extracts proved moderately to highly genotoxic in a dose-dependent manner, while apigenin-7$O$ - $\beta$-neohesperidoside (114), verbascoside (227), apigenin-7-O$\beta$-D-glucopyranoside (112), and apigenin (76) resulted as margin- ally genotoxic. Furthermore, the protective effect of all extracts and the isolated compounds was studied on nitrofurantoin (NF) induced damage. $\mathrm{MeOH}$, EtOAc, and $\mathrm{BuOH}$ extracts significantly decreased the induction factor of NF by $89.8 \%, 94.3 \%$, and $96.2 \%$, respectively, while compounds $76,112-114,139,223$, and 227 decreased the genotoxicity by a factor of 65 to $97 \%$.

\section{Insecticidal activity}

The hot water extract of the leaves of $B$. undulata from Jordan [273] was effective as a repellent agent against the sweet potato parasite Bemisia tabaci (Gennadius). A set of tomato leaves treated with the extract was compared with untreated tomato leaves, demonstrating a significant difference (analysis of variance test) in the number of insects that attacked each group. The same taxon, in the form of leaves brewed in hot water, showed acaricidal 
- Table 11 Occurrence of non-volatile metabolites in taxa of Ballota and Otostegia.

\begin{tabular}{|c|c|c|c|}
\hline Taxa & Diterpenes & Flavonoids & Others \\
\hline B. acetabulosa & $5,18,25,33$ & $\begin{array}{l}76,80,82,85,112,115,120,124,138 \\
145,146,160,162,164\end{array}$ & $185,188,190,194,201,230,235$ \\
\hline B. africana & 25 & & \\
\hline B. andreuzziana & 25 & $83,86,116,121,127$ & \\
\hline B. antalyensis & 5,18 & & \\
\hline B. arabica & 75 & 152,163 & $176,179,180,182,185,192,194,196,206,232$ \\
\hline B. aucheri & $\begin{array}{l}4,5,10,27,28,29 \\
30,31,34,44,46\end{array}$ & 159 & $185,188,190,194,201,230,235$ \\
\hline B. cinerea & $\begin{array}{l}12,13,14,15,16 \\
45,74\end{array}$ & $87,95,122,124,126,133$ & $\begin{array}{l}\text { 168-171, } 173,174,178,180,182,183,194 \text {, } \\
\text { 197, } 214,216,226,229,238 \text {; cetyl alcohol, glu- } \\
\text { cose, fructose, arabinose, palmitic acid, stearic } \\
\text { acid, oleic acid, oxalic acid, tartaric acid [176]; } \\
\text { hentriacontane, triacontane [116]; pentacosane, } \\
\text { octacosanol [154] }\end{array}$ \\
\hline B. cristata & $5,18,25$ & & $\begin{array}{l}\text { 204; linoleic acid, oleic acid, palmitic acid, } \\
\text { stearic acid, linolenic acid [190] }\end{array}$ \\
\hline B. deserti & $\begin{array}{l}2,3,19,17,20,21 \\
24,37-40\end{array}$ & $76,99,100,112-114,124,139$ & $180,182,183,189,194,196,223,227,233,236$ \\
\hline B. glandulosissima & & $81,88,90,97,106,107,108$ & \\
\hline B. hirsuta & 25 & $\begin{array}{l}76,78,80,81,85,89,104,106,112 \\
116,118,120,122,136,141,144,159\end{array}$ & $186,190,223,227$ \\
\hline B. hispanica & 51,52 & & \\
\hline B. inaequidens & 5,25 & $79,80,88,103,107-109,111$ & \\
\hline B. lanata & 5,32 & $\begin{array}{l}76,79,85,88,91,99,100,101,112, \\
116,122-125,128-132,134,139,140 \\
147-151,156,157\end{array}$ & $\begin{array}{l}176,180,182,183,185,186,188,199 \\
208-212,217,227,234\end{array}$ \\
\hline B. larendana & 5,18 & $112,116,137,139$ & \\
\hline B. latibracteolata & 18 & 80 & \\
\hline B macrodonta & 18 & $100,116,124,161,164-166$ & $\begin{array}{l}185,187,188,190-192,194-196,199,201 \\
202,204,207,231\end{array}$ \\
\hline B. nigra & $1,5,32,73$ & $76,80,85,91,96,142,143$ & $\begin{array}{l}\text { 174, 176, 180, 182, 185, 186, } 188,192,193 \text {, } \\
\text { 227, 200, 203, 215, 217-221, 223, } 224,226 \text {, } \\
\text { ascorbic acid, malic aconitic acid, citric acid, } \\
\text { a-linolenic acid, oleic acid, palmitic acid, stearic } \\
\text { acid [198] }\end{array}$ \\
\hline B. nigra subsp. anatolica & & 106 & $\begin{array}{l}\text { 236; } 10 \text {-undecenoic acid, myristic acid, palmito- } \\
\text { leic acid, palmitic acid, } 11,13 \text {-dimethyl-12-tetra- } \\
\text { decen-1-ol acetate, linoleic acid, oleic acid, lino- } \\
\text { lenic acid, stearic acid, arachidic acid, 7-methyl-6- } \\
\text { hexadecenoic acid, behenic acid [196] }\end{array}$ \\
\hline B. nigra subsp. foetida & $5,9,10,37,47$ & $103,106,108,112,159$ & $\begin{array}{l}213,217,237 \text {; palmitic acid, stearic acid, } \\
\text { octadecenoic acid, octadecadienoic acid, } \\
\text { octadecatrienoic acid [199] }\end{array}$ \\
\hline B. nigra f. uncinata. & 18 & & \\
\hline B. pilosa & & 76,139 & $175,180,182-184$ \\
\hline B. pseudodictamnus & $5,33,36$ & $79,112,120,145$ & $186,223,227$ \\
\hline B. pseudodictamnus subsp. lycia & 18,25 & & \\
\hline B. rotundifolia & 18,25 & $79,80,103$ & \\
\hline B. rupestris & $5,8,49$ & & $186,223,227$ \\
\hline B. saxatilis & $5,18,25,33$ & $80,103,108,109$ & \\
\hline B. saxatilis subsp. brachyodonta & 5,18 & $80,105,108$ & \\
\hline
\end{tabular}


- Table 11 Continued

\begin{tabular}{|l|l|l|l|}
\hline Taxa & Diterpenes & Flavonoids & Others \\
\hline B. undulata & $\mathbf{5 , 8 , 1 0 , 2 6}$ & $\begin{array}{l}90,102,105,107,112,116,119,124, \\
147\end{array}$ & $\begin{array}{l}183,215,217,222,223,225,227,239 ; \\
(-) \text {-carvone, [81] }\end{array}$ \\
\hline O. fruticosa & $\begin{array}{l}5,22,23,34,35, \\
41,42,46,50\end{array}$ & $84,98,117,120,159$ & $\begin{array}{l}167,174,176,180,185,192 ; \text { octacosane, } \\
\text { palmitic acid, linoleic acid, arachidic acid [175] }\end{array}$ \\
\hline O. integrifolia & 43,48 & & 183 ; pentatriacontane [100] \\
\hline O. limbata & $6,7,53-72$ & $92,93,135,153-155$ & \\
\hline O. persica & $56,59,71,72$ & $77,91,94,99,100,110,112,128,158$, & $\begin{array}{l}168,177,180,181,183,185,189,196 ; ~ g e r i a n o l, ~ \\
\text { eugenol, ceryl alcohol, hentiacontane [177] }\end{array}$ \\
\hline
\end{tabular}

activity against the spider mite Tetranychus urticae. A high mortality $(53 \%)$ was achieved by treating adults with the extract, while the eggs remained unaffected [274].

An insect repellent activity was also disclosed for 0 . integrifolia from Ethiopia [275]. The headspace of fresh leaves, dried leaves, and burned dried leaves were evaluated giving a repellent ratio of $29-56 \%$ in an elegant experimental setting developed by the author. This activity was shown to be associated with the presence of $\beta$-ocimene in the headspace blend.

The $n$-Hex extract of $O$. limbata (Pakistan) showed larvicidal activity against the banana parasite Drosophila melanogaster Meigen [276]. The crude solid extract was mixed with overripe banana at $2-6 \% \mathrm{w} / \mathrm{w}$ and larvae were let to feed. A concentration dependent mortality from 12 to $89 \%$ was observed. Also, a pupation reduction effect was established with a ratio of development dropping from 88 to $23 \%$, depending on the extract concentration (0.5$2.0 \%)$.

The Ac extract of $O$. persica leaves collected in Iran [277] was studied as a pesticide against Aphis fabae Scopoli, Aphis gossypii Glov., Myzus persicae Sulzer, and Tribolium castaneum Herbst. The maximum mortalities of individuals after $48 \mathrm{~h}$ of treatment at $80 \mu \mathrm{L} / \mathrm{mL}$ dose of extract were $55,58,88$, and $34 \%$, respectively. The prolongation of exposure time to $60 \mathrm{~h}$ did not appear to significantly improve the mortality rate.

An Ac extract of B. hirsuta (AP) from Spain [278] caused a significant growth inhibition in the T. castaneum larvae (29\%) accompanied by $20 \%$ mortality. This effect was related to the harvesting time, as the plant taken in November was active, while a sample collected in April was inactive.

\section{Effects on central nervous system}

The extract of $B$. limbata (Pakistan) leaves in $n$-buthanol was active as antitussive by reducing the $\mathrm{SO}_{2}$-induced cough in mice. The treatment of animals with $800 \mathrm{mg} / \mathrm{kg}$ b. w. of the extract caused the cough episodes to be reduced from 46 to 12 in 60 min; an efficacy analogous to that of the standard antitussive drugs codeine and dextromethorphan [279]. The toxicity test proved the extract to be inoffensive until the dose of $5000 \mathrm{mg} / \mathrm{g}$ b.w.

The brew (in hot water) of the AP of $B$. nigra subsp. anatolica from Turkey possess both antidepressant and anxiolytic activities in rats, determined with the forced swimming and the elevated plus-maze tests [280].
Two extracts of $O$. persica harvested in Iran were prepared by suspending the AP of the plant in $n-\mathrm{Hex}$ and $80 \% \mathrm{EtOH}$ and were tested for the alleviating effect in the opioid withdrawal syndrome [281], as this is a traditional use in Iran folk medicine. Male mice were intoxicated with morphine and the withdrawal signs (jumping, rearing, diarrhea, piloerection, tremor, and ptosis) were recorded after injection of naloxone in untreated animals, treated with clonidine $(0.2 \mathrm{mg} / \mathrm{kg}$ b. w.) and with increasing doses of both the extracts (500-1500 mg/kg b. w.). All of the clinical signs were reduced significantly to levels comparable to those obtained with clonidine at the maximum dose of utilized ethanolic extract. The $n$-Hex extract, however, was only able to reduce diarrhea.

An anticonvulsant effect was evidenced in the $\mathrm{MeOH}$ extract of O. persica [282], sustaining the traditional use of this plant in seizure management with scientific evidence. Convulsions were induced in mice with pentylenetetrazole, followed by an IP injection of $800 \mathrm{mg} / \mathrm{kg}$ b. w. which had a $93 \%$ protective effect, comparable to that of benzodiazepine.

The extract of AP of B. glandulosissima in water were investigated for their antinociceptive activity in mice by acetic acid-induced "writhing" and "tail-flick" tests [283]. A lethal dose of $8.85 \mathrm{~g} / \mathrm{kg}$ b. w. was determined. The extract, intraperitoneally administrated at 100 and $200 \mathrm{mg} / \mathrm{kg}$ b.w. doses, had promising antinociceptive activity, comparable to acetylsalicylic acid, utilized as a positive control.

An analgesic effect similar to paracetamol was obtained with the $\mathrm{MeOH}$ extract of $B$. deserti AP from Algeria [248] when administrated at $400 \mathrm{mg} / \mathrm{kg}$ b. w. to albino rats. Acetic acid induced abdominal writhes were reduced by $73 \%$.

\section{Metabolism control effects}

The extract of $B$. nigra from Jordan, obtained by brewing the AP in EtOH water $7: 3$, was proven to possess very interesting hypoglycemic activity both in healthy and in allossana-induced diabetic albino rats. After a single dose treatment of $400 \mathrm{mg} / \mathrm{g}$ b. w., the first group had a glucose blood concentration reduction from 96 to $62 \mathrm{mg} / \mathrm{dL}$ after $6 \mathrm{~h}$, while for the second group, the value dropped from 324 to $271 \mathrm{mg} / \mathrm{dL}$ [284]. In another study by the same authors [285], an analogous $B$. nigra preparation administrated at the same dose for $7 \mathrm{~d}$ was also effective in reducing hematic cholesterol (from 193 to $144 \mathrm{mg} / \mathrm{dL}$ ), triglycerides (from 97 to $83 \mathrm{mg} /$ $\mathrm{dL}$ ), and CK protein (from 431 to $348 \mathrm{IU} / \mathrm{L}$ ). 
- Table 12 Main compounds (> 3\%) of the essential oils from Ballota and Otostegia taxa.

\begin{tabular}{|c|c|c|c|c|}
\hline Taxa & & Origin & Main compounds & Ref. \\
\hline B. andreuzziana & $\mathrm{F}$ & G. Akhdar, Libya & caryophyllene (63.1), cis- $\gamma$-bisabolene (26.3), selinene (5.0) & [200] \\
\hline B. aucheri & AP & Fars, Iran & $\begin{array}{l}\alpha \text {-cadinol (21.0), dehydroaromadendrene (11.8), } \beta \text {-caryophyllene (8.1), carvone (6.4), } \\
\text { spathulenol (6.0), linalool (4.8), (Z)-methyl isoeugenol (4.1), } \alpha \text {-santalene (3.5) }\end{array}$ & [201] \\
\hline \multirow[t]{3}{*}{ B. deserti } & AP & Djelfa, Algeria & $\begin{array}{l}\text { germacrene D (45.7), } \beta \text {-bourbonene(4.0), } \alpha \text {-terpinolene (3.9), } \delta \text {-cadinene (3.8), } \\
\text { 1-octen-3-ol (3.7), } \alpha \text {-copaene (3.5) }\end{array}$ & [202] \\
\hline & AP & Ghardaïa, Algeria & $\begin{array}{l}\text { 9-methyl-undecene (21.3), } \delta \text {-cadinene (12.2), germacrene D (11.9), cis-phytol (7.7), } \\
\alpha \text {-cubebene (4.4) }\end{array}$ & [203] \\
\hline & AP & Algerian Sahara & $\begin{array}{l}\text { tetracosane (31.1), germacrene D (7.9), } \delta \text {-cadinene (6.5), } \alpha \text {-cadinol (6.3) t-cadinol (5.8), } \\
\beta \text {-elemene (3.8) }\end{array}$ & [204] \\
\hline B. hispanica & AP & Sicily, Italy & $\begin{array}{l}\alpha \text {-elemol (10.9), } \alpha \text {-ylangene (8.5), } \gamma \text {-dodecalactone (5.1), manoyl oxide (4.8), } \gamma \text {-eudesmol } \\
\text { (4.2), } \beta \text {-eudesmol (3.7), 1-pentadecene (3.7), germacrene } D(3.5) \text {, }\end{array}$ & [205] \\
\hline \multirow[t]{2}{*}{ B. lanata } & AP & Buryatia, Russia & $\begin{array}{l}\text { palmitic acid (14.3), camphor (12.4), } \alpha \text {-pinene (10.3), linalool (9.1), } \beta \text {-caryophyllene (8.3), } \\
\text { terpinen-4-ol (6.4), phytol (4.8), caryophyllene oxide (4.2), p-mentha-3-en-8-ol (3.3) }\end{array}$ & [29] \\
\hline & AP & Gobi, Mongolia & $\begin{array}{l}\text { camphor (14.4), } \alpha \text {-pinene, (11.3), terpinenol-4 (5.3), 6,10,14-trimethyl-2-pentadecanone } \\
\text { (4.7), } \beta \text {-caryophyllene (3.5), } \beta \text {-humulene (3.2), } \alpha \text {-thujene (3.1) }\end{array}$ & [206] \\
\hline \multirow[t]{2}{*}{ B. macedonica } & AP & Debar, Macedonia & germacrene D (24.6), (E)-caryophyllene (16.5), carotol (13.7), caryophyllene oxide (3.5) & [207] \\
\hline & AP & Prizren, Serbia & $\begin{array}{l}\text { carotol (52.1), germacrene D (8.6), (Z)-hex-3-en-1-ol (7.0), (E)-caryophyllene (6.5) } \\
\text { oct-1-en-3-ol (3.8) }\end{array}$ & [207] \\
\hline \multirow[t]{9}{*}{ B. nigra } & AP & Mazandaran, Iran & $\begin{array}{l}\text { caryophyllene oxide (7.9), epi- } \alpha \text {-muurolol (6.6), } \delta \text {-cadinene (6.5), } \alpha \text {-cadinol (6.3), } \\
\gamma \text {-amorphene (4.3), } \beta \text {-bourbonene (4.1), 6,10,14-trimethyl-2-pentadecanone (4.0), } \\
\text { (E)-caryophyllene (4.0), germacrene D (3.8), aromadendrene (3.4), } \gamma \text {-muurolene (3.2), } \\
\text { germacrene D-4-ol (3.2), } \alpha \text {-bisabolol (3.2), } \alpha \text {-amorphene (3.0) }\end{array}$ & [208] \\
\hline & S & Jadovnik Mt., Serbia & $\begin{array}{l}\beta \text {-caryophyllene (35.4), germacrene D (27.4), } \alpha \text {-humulene (7.4), } \delta \text {-cadinene (3.8), } \\
\text { (E)-phytol (2.5) }\end{array}$ & [209] \\
\hline & L & Jadovnik Mt., Serbia & $\beta$-caryophyllene (39.1), germacrene D (35.7), $\alpha$-humulene (10.4), (E)-phytol (3.8) & [209] \\
\hline & $\mathrm{R}$ & Jadovnik Mt., Serbia & $\begin{array}{l}\text { p-vinylguiacol (9.2), borneol (7.5), myrtenol (7.1), trans-pinocarveol (5.2), } \\
\text { 1-octen-3-ol (5.1), pinocarvone (4.4), 2-methyl-3-phenylpropanal (4.3), } \\
\text { p-cymen-8-ol (4.3), trans-carveol (3.5) }\end{array}$ & [209] \\
\hline & AP & Golestan, Iran & $\beta$-pinene (39.0), $\alpha$-pinene (34.5), sabinene (7.7), $\alpha$-phellandrene (4.1) & [210] \\
\hline & corollas & Kharkov, Ukraine & $\begin{array}{l}\text { palmitic acid }(573)^{\mathrm{a}}, 2,2,6 \text {-trimethyl-4-methylene- } 2 \mathrm{H}-\text { pyran }(172)^{\mathrm{a}} \text {, hexahydrofarnesylace- } \\
\text { tone }(167)^{\mathrm{a}} \text {, miristic acid }(100)^{\mathrm{a}} \text {, caryophyllene oxide }(57)^{\mathrm{a}} \text {, pentadecanoic acid }(50)^{\mathrm{a}} \text {, } \\
\text { palmitoliec acid }(40)^{\mathrm{a}} \text {, germacrene } \mathrm{D}(40)^{\mathrm{a}} \mathrm{mg} / \mathrm{kg}\end{array}$ & [211] \\
\hline & calyx & Kharkov, Ukraine & $\begin{array}{l}\text { palmitic acid }(1620)^{\mathrm{a}} \text {, dodecanal }(519)^{\mathrm{a}} \text {, palmitoliec acid }(306)^{\mathrm{a}} \text {, miristic acid }(271)^{\mathrm{a}} \text {, } \\
\text { pentadecanoic acid }(182)^{\mathrm{a}} \text {, lauric acid }(67)^{\mathrm{a}} \text {, trans-isoelemicin }(67)^{\mathrm{a}} \text {, hexahydrofarnesylace- } \\
\text { tone }(60)^{\mathrm{a}} \text {, pentadecene }(54)^{\mathrm{a}} \text {, methyleugenol }(40)^{\mathrm{a}} \mathrm{mg} / \mathrm{kg}\end{array}$ & [211] \\
\hline & L & Kharkov, Ukraine & $\begin{array}{l}\text { palmitic acid }(656)^{\mathrm{a}} \text {, palmitoliec acid }(197)^{\mathrm{a}} \text {, miristic acid }(187)^{\mathrm{a}} \text {, pentadecanoic acid }(121)^{\mathrm{a}} \text {, } \\
\text { farnesylacetone }(69)^{\mathrm{a}} \text {, dihydroactinidiolide }(44)^{\mathrm{a}} \mathrm{mg} / \mathrm{kg}\end{array}$ & [211] \\
\hline & S & Kharkov, Ukraine & $\begin{array}{l}\text { methylsalicilate (313) a palmitic acid (130)a , 2,2,6-trimethyl-4-methylene-2H-pyran (42)a, } \\
\text { miristic acid }(42)^{\mathrm{a} a \mathrm{mg}} / \mathrm{kg}\end{array}$ & [211] \\
\hline \multirow[t]{4}{*}{$\begin{array}{l}\text { B. nigra L. subsp. } \\
\text { anatolica }\end{array}$} & AP & Mazandaran, Iran & $\begin{array}{l}\text { germacrene } \mathrm{D} \text { (18.1), nerolidol epoxyacetate (15.4), sclareol oxide (12.1), linalyl acetate } \\
\text { (11.5), } \beta \text {-caryophyllene (10.5), spathulenol (9.0), linalool (5.2), longipinene epoxide (4.7) }\end{array}$ & [212] \\
\hline & $\mathrm{F}$ & Çamlica, Turkey & $\begin{array}{l}\text { hexenal (21.2), (E)- } \beta \text {-caryophyllene (10.0), germacrene D (7.8), cis-3-hexene-1-ol (6.8), } \\
\text { pentanal (6.9), limonene (5.2), (E)-2-hexenal (3.0) }\end{array}$ & [213] \\
\hline & AP & Muğla, Turkey & $\begin{array}{l}\text { hexadecanoic acid (40.9), } \beta \text {-bisabolene (13.4), hexahydrofarnesyl acetone (7.9), } \\
\text { 1-isobutyl-4-isopropyl-2,2-diemethyl succinate (6.6), } \beta \text {-eudesmol (3.5) }\end{array}$ & [214] \\
\hline & AP & Western Turkey & $\begin{array}{l}\text { 1-hexacosanol (26.7), caryophyllene oxide (9.3), germacrene-D (9.3), } \alpha \text {-selinene (8.7), } \\
\text { Z-8-octadecen-1-ol acetate (7.1), 2,5-di-tertoctyl-p-benzoquinone (7.3), } \\
\text { arachidic acid (6.0), tetracosane (4.5), heneicosane (4.4), heptacosane (4.3), } \\
\text { 2-methyl-1-hexadecanol (3.3), octadecane (3.0), butyl phthalate (3.0) }\end{array}$ & [196] \\
\hline
\end{tabular}


- Table 12 Continued

\begin{tabular}{|c|c|c|c|c|}
\hline Taxa & & Origin & Main compounds & Ref. \\
\hline \multirow[t]{6}{*}{$\begin{array}{l}\text { B. nigra subsp. } \\
\text { foetida }\end{array}$} & $\mathrm{AP}$ & Pisa, Italy & $\begin{array}{l}\beta \text {-caryophyllene (25.1), germacrene D (24.2), 1-octen-3-ol (7.3), (E)-2-hexenal (6.1), } \\
\alpha \text {-humulene (4.3), caryophyllene oxide (4.2) }\end{array}$ & [215] \\
\hline & AP & Urbino, Italy & $\begin{array}{l}\beta \text {-caryophyllene (20.0), germacrene D (18.0), caryophyllene oxide (15.0), } \\
\text { 1-octen-3-ol (6.8), (E)-2-hexenal }(6.1), \alpha \text {-humulene (4.5), } \beta \text {-bourbonene (3.2) }\end{array}$ & [216] \\
\hline & $\begin{array}{l}\text { AP } \\
\text { flowering }\end{array}$ & Urbino, Italy & $\begin{array}{l}\beta \text {-caryophyllene (22.6), caryophyllene oxide (18.0), germacrene D (16.5), } \\
(E) \text {-2-hexenal (6.5), 1-octen-3-ol (5.5) }\end{array}$ & [217] \\
\hline & $\begin{array}{l}\text { AP } \\
\text { fruiting }\end{array}$ & Urbino, Italy & $\begin{array}{l}\beta \text {-caryophyllene (21.8), caryophyllene oxide (20.5), germacrene D (13.1), } \\
(E) \text {-2-hexenal (11.2), } \beta \text {-pinene (4.4), limonene (4.1), 1-octen-3-ol (3.5), linalool (3.5) }\end{array}$ & [217] \\
\hline & $\mathrm{AP}$ & Nis, Serbia & $\begin{array}{l}\text { (E)-phytol (56.9), germacrene D (10.0), } \beta \text {-caryophyllene (4.7), caryophyllene oxide (3.6), } \\
\text { (E)- } \beta \text {-ionone (3.4) }\end{array}$ & [207] \\
\hline & $\mathrm{AP}$ & Brac, Croatia & $\begin{array}{l}\text { germacrene D (23.1), } \beta \text {-caryophyllene (20.3), caryophyllene oxide (6.2), caryophylladienol I } \\
\text { (3.3), (E)-2-hexenal (3.1), hexadecanoic acid (3.1), } \alpha \text {-humulene (3.0) }\end{array}$ & [218] \\
\hline $\begin{array}{l}\text { B. nigra subsp. } \\
\text { kurdica }\end{array}$ & $\mathrm{AP}$ & Kurdistan, Iran & $\begin{array}{l}\text { caryophyllene oxide (39.4), } \beta \text {-caryophyllene (24.9), germacrene D (7.6), 1-undecene (4.2), } \\
\text { isoaromadendrene epoxide (3.2) }\end{array}$ & [219] \\
\hline B. nigra f. uncinata & $\mathrm{AP}$ & Konya, Turkey & $\begin{array}{l}\text { caryophyllene oxide ( } 21.2) \text {, hexadecanoic acid (19.9), } \beta \text {-caryophyllene (18.9), germacrene } \\
\text { D (4.6), hexahydrofarnesyl acetone (4.4), spathulenol (4.2), caryolphyllenol II (3.8); } \\
\text { bicyclogermacrene (3.7) }\end{array}$ & [214] \\
\hline $\begin{array}{l}\text { B. pseudo- } \\
\text { dictamnus }\end{array}$ & $\mathrm{AP}$ & Crete, Greece & $\begin{array}{l}\text { caryophyllene oxide (22.4), phytol (11.9), } \gamma \text {-muurolene (11.4), (E)-caryophyllene (10.7), } \\
\alpha \text {-copaene (6.1), } \beta \text {-cucubene (5.3), hexahydrofarnesyl acetone (3.5) }\end{array}$ & [220] \\
\hline \multirow[t]{2}{*}{ B. saxatilis } & AP & Amman, Jordan & $\begin{array}{l}\text { linalool (14.6), caryophyllene oxide (11.0), acorenone (9.3), } \beta \text {-caryophyllene (7.9), } \\
\text { germacrene D (7.6), 1-octen-3-ol (3.6), } \beta \text {-bourbonene (3.0) }\end{array}$ & [215] \\
\hline & $\mathrm{AP}$ & Kfardin, Lebanon & $\begin{array}{l}\text { linalool (11.2), (E)- } \beta \text {-caryophyllene (8.8), caryophyllene oxide (6.3), (E)-2-hexenal (5.6), } \\
\text { hexadecanoic acid (4.9), (Z,Z)-9,12-octadecadienoic acid (3.4) }\end{array}$ & [218] \\
\hline $\begin{array}{l}\text { B. saxatilis subsp. } \\
\text { brachyodonta }\end{array}$ & $\mathrm{AP}$ & Mersin, Turkey & $\begin{array}{l}\text { (E)- } \beta \text {-caryophyllene (23.9), epi-bicyclosesqui-phellandrene (20.2), caryophyllene oxide } \\
\text { (10.5), } \gamma \text {-elemene (5.5), thymol (4.1) }\end{array}$ & [221] \\
\hline Ballota schimperi & $\mathrm{L}$ & Yemen & $\begin{array}{l}\text { T-cadinol (9.3), } \beta \text {-caryophyllene (8.8), bornyl formate (5.2), myrtenyl formate (3.8), } \\
\text { spathulenol (3.2), } \beta \text {-selinene (3.0) }\end{array}$ & [222] \\
\hline B. sechmenii & & Turkey & linalool (5) (ratio (+)-linalool: (-)-linalool = 27:73) & [223] \\
\hline \multirow[t]{2}{*}{ B. undulata } & AP & Naur, Jordan & $\begin{array}{l}\text { germacrene D (19.1), bicyclogermacrene (11.6), viridiflorol (6.0), 1-octen-3-ol (3.5), } \\
\text { epi-10-y-eudesmol (3.1) }\end{array}$ & {$[215]$} \\
\hline & AP & Kfardin, Lebanon & $\begin{array}{l}\text { germacrene D (16.0), bicyclogermacrene (10.4), 9,12-octadecadienoic acid (5.3), } \\
\text { hexadecanoic acid (4.5), dihydroactinidiolide (3.4) }\end{array}$ & {$[218]$} \\
\hline \multirow[t]{2}{*}{ O. fruticosa } & $\begin{array}{l}\text { AP culti- } \\
\text { vated }\end{array}$ & El-Mansoura, Egypt & thymol (43.7), $\gamma$-terpinene (16.4), $p$-cymene (12.4), (E)- $\beta$-caryophyllene (9.5) & {$[224]$} \\
\hline & AP & Sinai, Egypt & $\begin{array}{l}\text { caryophyllene oxide (60.8), } \beta \text {-bisabolene (9.2), 4-decyne (5.1), } \alpha \text {-cis-bergamotene (4.4), } \\
\beta \text {-bourbonene (4.2), linalyl acetate (4.2) }\end{array}$ & {$[175]$} \\
\hline O. integrifolia & Ls & North Shoa, Ethiopia & $\begin{array}{l}\alpha \text {-pinene (31.3), 1-octen-3-ol (11.8), } \beta \text {-caryophyllene (11.3), linalool (6.6), cis- } \beta \text {-ocimene } \\
\text { (5.9), germacrene D (3.3) }\end{array}$ & {$[56]$} \\
\hline \multirow[t]{2}{*}{ O. michauxii } & AP & Zagros, Iran & $\begin{array}{l}\text { caryophyllene oxide (20.1), trans-verbenol (10.2), linalool (5.3) and humulene epoxide II } \\
\text { (4.6) }\end{array}$ & {$[225]$} \\
\hline & AP & Fars, Iran & $\begin{array}{l}\text { dillapiole (23.9), 2-methylbenzofuran (12.9), } \alpha \text {-pinene (8.1), } \delta \text {-cadinene (6.1), } \\
\text { 1-octen-3-ol (4.9), caryophyllene oxide (4.8), linalool (4.5), (E)- } \beta \text {-caryophyllene (3.6) }\end{array}$ & [226] \\
\hline \multirow[t]{6}{*}{ O. persica } & AP & Fars, Iran & dillapiole (43.1), trans-verbenol (9.6), hexadecanoic acid (5.7), isospathulenol (4.5) & [227] \\
\hline & $\mathrm{L}$ & Sistan, Iran & $\begin{array}{l}\text { hexahydrofarnesyl acetone (14.3), trans-verbenol (10.2), geranyl acetone (6.5), } \\
\text { pentadecane (5.9), hexadecane (5.9), } \alpha \text {-pinene (4.5), trans-anethole (4.5), verbenone (3.5), } \\
\text { 1-octen-3-ol (3.0) }\end{array}$ & [228] \\
\hline & $\mathrm{F}$ & Sistan, Iran & $\begin{array}{l}\alpha \text {-pinene (13.6), trans-verbenol (9.2), linalool (6.8), hexadecane (5.5), caryophyllene oxide } \\
\text { (4.8), pentadecane (4.6), trans-carveol (4.0), 1-octen-3-ol (3.8), geranyl acetone (3.7), } \\
\text { heptadecane (3.3) }\end{array}$ & [228] \\
\hline & $\begin{array}{l}\text { flowering } \\
\text { AP }\end{array}$ & Kerman. Iran & $\begin{array}{l}\text { hexadecanoic acid (31.7), pentacosane (29.5), } \alpha \text {-copaene-8-ol (5.9), hexadecanoic acid } \\
\text { methylester (4.8), caryophyllene oxide (3.8), trans-damascenone (3.7) }\end{array}$ & {$[228]$} \\
\hline & $\mathrm{F}$ & Kerman. Iran & $\alpha$-pinene (17.2), 1-octen-3ol (13.4), cubenol (7.3) & [229] \\
\hline & fruits & Kerman. Iran & diisooctyl phthalate (45.0), hexadecanoic acid (11.1) & [229] \\
\hline
\end{tabular}


- Table 13 The antioxidant activity of Ballota and Otostegia taxa.

\begin{tabular}{|c|c|c|c|c|}
\hline Species & Origin & $\begin{array}{l}\text { Sample preparation } \\
\text { (plant part-solvent) }\end{array}$ & Test & Ref. \\
\hline $\begin{array}{l}\text { B. acetabulosa, B. antalyanse, B. cristata, } \\
\text { B. glandulosissima, B. inaequidens, } \\
\text { B. larendana, B. latibracteolata, B. macro- } \\
\text { donta, B. nigra ssp. anatolica, B. nigra ssp. } \\
\text { foetida, B. nigra, B. nigra ssp. uncinata, } \\
\text { B. pseudodictamnus ssp. lycia, B. rotundi- } \\
\text { folia, B. saxatilis ssp. brachyodonta, } \\
\text { B. saxatilis }\end{array}$ & Turkey & L: EtOAc, MeOH, W & $\begin{array}{l}\text { FRAP (\% inhib.): B. antalyense (1.34) MeOH extr., B. saxa- } \\
\text { tilis ssp. brachyodonta MeOH extr. (1.28) B. saxatilis } \mathrm{MeOH} \\
\text { extr. (1.12); B. antalyense W extr. (1.24) }\end{array}$ & [230] \\
\hline $\begin{array}{l}\text { B. antalyense, B. macrodonta, B. glandulo- } \\
\text { sissima, B. larendana, B. pseudodictam- } \\
\text { nus, B. nigra ssp. anatolica, B. rotundifolia, } \\
\text { B. saxatilis ssp. brachyodonta, B. saxatilis }\end{array}$ & Turkey & L: EtOH/W $3: 1$ & $\begin{array}{l}\text { Superoxide anion formation quenching (SAFQ): } \\
\mathrm{IC}_{50} 0.50 \text { to } 0.87 \mathrm{mg} / \mathrm{mL} \text {; Liver rats lipid peroxidation } \\
\text { (LO): not significant }\end{array}$ & [231] \\
\hline $\begin{array}{l}\text { B. antalyense, B. macrodonta, } \\
\text { B. glandulosissima }\end{array}$ & Turkey & L: EtOH/W $3: 1$ & SAFQ: $0.50,0.51,0.51$ & [231] \\
\hline $\begin{array}{l}\text { B. inaequidens, B. glandulosissima, } \\
\text { B. saxatilis, B. macrodonta, B. antalyense }\end{array}$ & Turkey & L: EtOH/W $3: 1$ & $\mathrm{LO}(\mathrm{mg} / \mathrm{mL}): 12$ to $20 \mathrm{mg} / \mathrm{mL}$ & [231] \\
\hline B. inaequidens, B. glandulosissima & Turkey & L: EtOH/W $3: 1$ & LO: $(\mathrm{mg} / \mathrm{mL}) 12$ and 15 & [231] \\
\hline B. aucheri & Pakistan & AP: $70 \% \mathrm{MeOH}$ & DPPH: IC ${ }_{50}(\mu \mathrm{g} / \mathrm{mL}) 2.23$ & [232] \\
\hline B. cinerea & India & $\begin{array}{l}\text { AP: EtOH, then partition in } \mathrm{CHCl}_{3} \text {, } \\
\text { EtOAc, } n \text {-BuOH, W }\end{array}$ & $\begin{array}{l}\text { DPPH: IC }{ }_{50}(\mu \mathrm{g} / \mathrm{mL}) \text { 85-661; FRAP }(\mathrm{mmolFe} / \mathrm{g}) 0.14- \\
0.59 ; \text { ABTS IC }_{50}(\mu \mathrm{g} / \mathrm{mL}) \text { 60-840; ORAC (TEAC mM) } \\
8.55-36.0\end{array}$ & [233] \\
\hline B. deserti & Tunisia & $\begin{array}{l}\text { L: } \mathrm{MeOH} \text {, then partition in pet. } \\
\text { ether, } \mathrm{CHCl}_{3} \text {, EtOAc, } \mathrm{BuOH}\end{array}$ & $\begin{array}{l}\mathrm{DPPH}: \mathrm{IC} 50(\mathrm{mg} / \mathrm{mL}) 0.85-2.50 \text {; ABTS: } \mathrm{IC} 50(\mathrm{mg} / \mathrm{mL}) \\
0.35-13 \text {, pet. Ether inactive }\end{array}$ & [25] \\
\hline B. deserti & Algeria & EO & DPPH: IC $5_{50} \mu \mathrm{g} / \mathrm{mL} 35.9$ & [204] \\
\hline B. deserti & Algeria & $\begin{array}{l}\text { AP: } \mathrm{CH}_{2} \mathrm{Cl}_{2}, \mathrm{MeOH} \text {, then isolat. } \\
\text { compds: } 114,223,227\end{array}$ & ABTS: $\mathrm{IC}_{50}(\mathrm{~g} / \mathrm{mmol})$ 0.14-3.50 & [90] \\
\hline B. deserti & Algeria & $\begin{array}{l}\text { AP: } \mathrm{CH}_{2} \mathrm{Cl}_{2}, \mathrm{MeOH} \text {, then isolat. } \\
\text { compds: } 76,112-114,139,223 \text {, } \\
227\end{array}$ & $\begin{array}{l}\mathrm{ABTS}: \mathrm{EC}_{50}(\mathrm{~mol} \%) 0.09->0.50 ; \mathrm{CUPRAC}: \mathrm{E} \%(\mathrm{~L} / \mathrm{mol} / \mathrm{cm}) \\
0.01-0.80 ; \mathrm{DPPH}: \mathrm{EC}_{50} \text { (mol \%) } 0.39->1.5,9 \text { and } \\
12 \text { oxidant or pro-oxidant }\end{array}$ & [89] \\
\hline B. hirsuta & Algeria & $\begin{array}{l}\mathrm{L}: \mathrm{W} / \mathrm{MeOH} 1: 1 \text {, then partition in } \\
\mathrm{EtOAc}, \mathrm{CHCl}_{3}, n-\mathrm{BuOH}\end{array}$ & $\begin{array}{l}\text { DPPH IC } \text { Fo }(\mathrm{mg} / \mathrm{mL}): 0.35 \text { extr., } 0.07 \text { EtOAc, } 0.26 \mathrm{CHCl}_{3} \text {, } \\
0.12 n-\mathrm{BuOH}\end{array}$ & [234] \\
\hline B. nigra & ex vitro & Shoots: $\mathrm{MeOH}$ & $\begin{array}{l}\mathrm{DPPH} \mathrm{EC}_{50}(\mathrm{mg} / \mathrm{mL}): 56.0-202.6, \text { FRAP }(\mu \mathrm{mol} / \mathrm{g}) 331.5- \\
\text { 642.4; LPO (\% inhb.) 20.97-36.05 }\end{array}$ & [235] \\
\hline B. nigra & $\begin{array}{l}\text { Czech } \\
\text { Republic }\end{array}$ & $\mathrm{L}: \mathrm{W}$ & $\begin{array}{l}\text { DPPH IC }_{25}(\mu \mathrm{g} / \mathrm{mL}) 4.81 ; \mathrm{X} / \mathrm{XO}(\mu \mathrm{g} / \mathrm{mL}) 14.6 ; \mathrm{HClO} \text { scav. } \\
80 \% \text { at } 500 \mu \mathrm{g} / \mathrm{mL} \text {; NO scav. IC25 }(\mu \mathrm{g} / \mathrm{mL}) 122\end{array}$ & [185] \\
\hline \multirow[t]{2}{*}{ B. nigra } & \multirow[t]{2}{*}{ France } & \multirow[t]{2}{*}{$\begin{array}{l}\text { L: } 50 \% \text { EtOH, then isolat. compds: } \\
186,220,221,223,227\end{array}$} & $\begin{array}{l}\mathrm{O}_{2}^{2-} \text { scav: } \mathrm{IC}_{50}(\mu \mathrm{g} / \mathrm{mL}) 30.6-149.0 ; \mathrm{H}_{2} \mathrm{O}_{2} \text { scav.: } \\
\mathrm{IC}_{50}(\mu \mathrm{g} / \mathrm{mL}) 2.3-11.2 ; \mathrm{HClO} \text { scav.: } \mathrm{IC}_{50}(\mu \mathrm{g} / \mathrm{mL}) 1.5-9.3 ; \\
\text { OH rad. scav.: } \mathrm{IC} C_{50}(\mu \mathrm{g} / \mathrm{mL}) 26.7-64.7 .\end{array}$ & [236] \\
\hline & & & LDL-Ox: $\mathrm{ED}_{50}(\mu \mathrm{M}) 1.0-9.5$ & [237] \\
\hline B. nigra ssp. anatolica & Turkey & WP: PE, Ac, MeOH, W & ABTS (\% inhib. at $100 \mathrm{mg} / \mathrm{mL}$ ): $72-80$ & [196] \\
\hline B. rotundifolia & Turkey & AP: $\mathrm{MeOH}$ & DPPH $(\mu \mathrm{g} / \mathrm{mg})$ 138.0; LPO (\% inhib.): 35.97 & [238] \\
\hline B. undulata & Jordan & $\begin{array}{l}\text { L: MeOH: compds: } 116,119,147 \text {, } \\
222,223,225,227,239\end{array}$ & ABTS, TEAC (mM) 0.68-1.67 & [150] \\
\hline B. pseudodictamnus, B. acetabulosa & Grecia & $\mathrm{L}: \mathrm{MeOH}$ & Activity equal to $\alpha$-tocopherol in Umezawa essay & [239] \\
\hline O. integrifolia & Ethiopia & L: EtOAc, MeOH & DPPH: 82.9 MeOH, 32.7 EtOAc; & [240] \\
\hline O. integrifolia & Ethiopia & EO & DPPH: $\mathrm{EC}_{50}(\mu \mathrm{L} / \mathrm{mL}) 5.32$ & [56] \\
\hline O. limbata & Pakistan & WP: $\mathrm{MeOH}$ & $\begin{array}{l}\text { DPPH: IC } 50 \text { (mg/mL) 13.53-129.52; FRAP, (mM/mL) } \\
\text { 88.86-334.27; LPO: ([\% inhib.] 12.67-61.76). }\end{array}$ & [241] \\
\hline O. limbata & Pakistan & $\begin{array}{l}\text { AP: } \mathrm{MeOH} \text { then solubilization } \\
\text { in } n-\mathrm{Hex}, \mathrm{CHCl}_{3}, \mathrm{EtOAc}, \mathrm{BuOH} \text {, } \\
\mathrm{MeOH}, \mathrm{W}\end{array}$ & $\begin{array}{l}\text { DPPH: } \text { EC }_{50}(\mu \mathrm{g} / \mathrm{mL}) \text { 60-350; FRAP }(\mathrm{mmol} / \mathrm{mg}) \text { 5-41; } \\
\text { ABTS, TEAC }(\mu \mathrm{mol} / \mathrm{g}) \text { 30-139 }\end{array}$ & [242] \\
\hline
\end{tabular}


- Table 13 Continued

\begin{tabular}{|c|c|c|c|c|}
\hline Species & Origin & $\begin{array}{l}\text { Sample preparation } \\
\text { (plant part-solvent) }\end{array}$ & Test & Ref. \\
\hline O. persica & Iran & $\begin{array}{l}\text { WP: } \mathrm{MeOH} \text {, then solubilization in } \\
n-\mathrm{Hex} \text { and } \mathrm{CHCl}_{3}\end{array}$ & LPO (\% inhib.) 95.87 MeOH, 2.5 nHex., $1.9 \mathrm{CHCl}_{3}$ & [158] \\
\hline O. persica & Iran & EO & DPPH: IC $50(\mathrm{mM}) 9.76$ & [228] \\
\hline O. persica & Iran & $\begin{array}{l}\text { EO in flowering } \\
\text { EO in fruiting }\end{array}$ & DPPH: IC 50 ( $\mu \mathrm{g} / \mathrm{mL})$ 19.8, 29.2; LPO (\% inhib.) 93.8, 63.0 & [229] \\
\hline O. persica & Iran & AP: $\mathrm{MeOH}$ & LPO (\% inhib.) 95.87 & [160] \\
\hline O. persica & Iran & AP: $\mathrm{MeOH}$, EtOAc & DPPH: IC $50.49 \mathrm{mg} / \mathrm{mL}$ for both & [141] \\
\hline O. persica & Iran & $\begin{array}{l}\mathrm{L}: 70 \% \mathrm{EtOH}_{\text {, then partition in PE, }} \\
\text { EtOAc, } \mathrm{CHCl}_{3}, n-\mathrm{BuOH}, \mathrm{MeOH}\end{array}$ & DPPH: $\mathrm{IC}_{50}(\mu \mathrm{g} / \mathrm{mL}) 170$ (EtOH) to 1580 (pet. Et.) & [243] \\
\hline
\end{tabular}

Similar hypolipidemic effects were observed in rabbits treated with the AP extract, in EtOH/water 7:3, of $B$. undulata from Jordan [286]. Two animal groups were treated with $400 \mathrm{mg}$ of cholester$\mathrm{ol} / \mathrm{kg}$ b.w. per day dissolved in $5 \mathrm{~mL}$ of coconut oil for $120 \mathrm{~d}$; in 1 group $1.2 \mathrm{~g} / \mathrm{kg} \mathrm{b.w}$. per day of the extract were added to the diet. A strong difference in lipidemic parameters was observed for the 2 groups: total cholesterol 807 versus 104, HDL 246 versus 40 , phospholipids 252 versus 112 , triglycerides 259 versus 74 . Despite their scientific relevance, in our opinion, the eventual transfer of these findings on human trials might be strongly inhibited by the evident difficulty to orally administer a dose of $84 \mathrm{~g} /$ day of plant extract to an average weight human.

A potent antidiabetic effect was identified for the ethanolic extract of the O. persica AP from Iran [287]. The hematic glucose level of STZ-induced diabetic rats was normalized when animals received 200 to $500 \mathrm{mg} / \mathrm{kg}$ b. w. per day of the extract. At the maximum dose, the glucose level was lowered from 405-420 to 170$230 \mathrm{mg} / \mathrm{dL}$, depending on the control time. Similar results were obtained by other authors [288] with the methanolic extract of this plant, also collected in Iran. In this case, the glucose level reduction observed in rat blood was accompanied by an increase in the insulin secretion in $C 187$ pancreatic $\beta$-cells. Additionally, a reduction in lipidic oxidation was proved by the decrease in MDA values and the increase in GSH values. The ethanolic extract of $O$. persica was demonstrated to possess protective effects by preventing renal damage induced by ischemia/reperfusion induced in diabetic rats [289]. The hematic renal function indicators were ameliorated after treatment with $300 \mathrm{mg} / \mathrm{kg}$ b. w. of the extract for $2 \mathrm{wk}$ : urea from 67.6 to 36.1 , creatinine from 2.32 to 1.32 , glucose from 378.6 to 147.2. Furthermore, kidney resection and tissue evaluation of the oxidative stress parameters (MDA, MPO, NO, SOD, and CAT) evidenced a beneficial effect in $O$. persica treated animals.

The antidiabetic activity of the AP of $O$. persica were assessed in different factions of the crude extract, following their solubility in $\mathrm{PE}, \mathrm{CHCl}_{3}$, EtOAc, $n-\mathrm{BuOH}$, and $\mathrm{MeOH}$ [141]. The antioxidant activity was measured by DPPH method and was correlated to antidiabetic activity in mice. $\mathrm{MeOH}$ extract was effective in reducing hematic glucose with a $300 \mathrm{mg} / \mathrm{kg}$ b.w. dose. Both $\mathrm{MeOH}$ an EtOAc extract showed antioxidant activity with $\mathrm{IC}_{50}$ of $0.49 \mathrm{mg} /$ $\mathrm{mL}$ for both. Finally, 4 compounds were isolated from the active extracts: chrysoeriol (91) from EtOAc, 6-methylapigenin (77), api-
genin-7-O- $\beta$-D-glucopyranoside (112), and echinaticin (138) from the $\mathrm{MeOH}$ one.

The aerial part of $O$. persica also showed a potent antidiabetic effect when extracted in water at $40^{\circ} \mathrm{C}$ [290]. Fasting blood sugar, insulin, and HOMA.IR (homeostasis model assessments for insulin resistance) were evaluated in STZ-induced diabetic mice after 10 , 20 , and $30 \mathrm{~d}$ of administration of up to $400 \mathrm{mg} / \mathrm{kg} \mathrm{b}$. w. of the extract. The indicator improvements were comparable to those reported in other studies, and total cholesterol and triglycerides were also significantly reduced: 95 versus $75 \mathrm{mg} / \mathrm{dL}$ for the former and 203 versus 71 for the latter. A reduction in the number and the mass of pancreatic $\beta$-cells was evidenced by histopathological visualization techniques.

The antidiabetic effect of O. persica (AP extracted in $\mathrm{EtOH} / \mathrm{H}_{2} \mathrm{O}$ $1: 1)$ was also studied by stereological analysis of pancreas tissue in diabetic (STZ induction) Sprague-Dawley rats [291]. The oral administration of $500 \mathrm{mg} / \mathrm{kg}$ b.w. reduced blood glucose levels and insulin production, as reported in many other references. After $1 \mathrm{mo}$, the animals were sacrificed and pancreatic tissue was analyzed. A hypertrophic change in the remaining $\beta$-cells of the diabetic group was observed, accompanied by a reduction in pancreatic islet volume. These phenomena were significantly reduced in the animals treated with the extract.

B. aucheri extract (AP in $70 \% \mathrm{MeOH}$ ) [232] was effective in reducing postprandial hematic glucose increment in type II diabetic rats, while it was ineffective in type I diabetic animals. This antidiabetic activity was associated with a notable antioxidant activity determined in this sample ( $\triangleright$ Table 13 ).

Bone damage such as osteoporosis may constitute an important comorbidity in patients affected by mellitus diabetes. The aqueous extract of $O$. persica (Iran) was proven to act as a protection from bone damage in STZ treated diabetic rats [292]. Rats was treated orally with $200-450 \mathrm{mg} / \mathrm{kg}$ b.w. for $29 \mathrm{~d}$. Then the left femoral and tibiofibular bones were dissected and evaluated histomorphometrically, while the right-side bones were removed for ash weight determination. The plant extract was able to significantly reverse the epiphyseal and metaphyseal trabecular width reduction observed in untreated animals. Additionally, the epiphyseal bone area/tissue were normalized with the utilization of the minimum extract dose. Ash weight was significantly lower in animals treated at $450 \mathrm{mg}$ dose. 
- Table 14 Antibacterial activity of Ballota and Otostegia taxa.

\begin{tabular}{|c|c|c|c|c|c|}
\hline Species & Origin & $\begin{array}{l}\text { Sample preparation } \\
\text { (plant part: solvent) }\end{array}$ & Test & Target & Ref \\
\hline B. acetabukosa & Turkey & L: EtOH $80 \%, W$ & $\begin{array}{l}\text { MIC, MBC }(\mu \mathrm{g} / \mathrm{mL}): 0.4- \\
1.6 ; 3.2-12.5(\mathrm{EtOH}), 0.8- \\
3.2,6.3-12.5 \mathrm{~W}\end{array}$ & S. aureus & [249] \\
\hline B. acetabukosa & Turkey & L: EtOH & $\begin{array}{l}\text { MIC }(\mathrm{mg} / \mathrm{mL}) 32-1024 \\
\mathrm{MBC}(\mu \mathrm{g} / \mathrm{mL}) 64-1024\end{array}$ & $\begin{array}{l}\text { E. faecalis, E. coli, P. mirabilis, K. pnemoniae, } \\
\text { P. aeruginosa }\end{array}$ & [250] \\
\hline B. africana & S. Africa & L: $\mathrm{MeOH}, \mathrm{W}$ & MIC $(\mu \mathrm{g} / \mathrm{mL}) 438$ & K. pneumoniae, A. nauplii & [251] \\
\hline B. andreuzziana & Libya & WP: EtOAc, $\mathrm{CHCl}_{3}, \mathrm{BuOH}, \mathrm{W}$ & $\begin{array}{l}\text { AD at } 50,100,150 \mathrm{mg} / \mathrm{mL} \\
(\mathrm{mm}) 7-11\end{array}$ & S. aureus, B. subtilis, My phlei & [148] \\
\hline B. deserti & Algeria & $\begin{array}{l}\mathrm{EO} \\
\mathrm{L}: \mathrm{MeOH}\end{array}$ & $\begin{array}{l}\text { MIC biofilm formation EO } \\
(\mu \mathrm{L} / \mathrm{mL}) 25-80 ; \mathrm{MeOH} \\
(\mathrm{mg} / \mathrm{mL}) 3.25-25\end{array}$ & $\begin{array}{l}\text { S. aureus (ATCC } 25923 \text {, ATCC } 6538 \text {-P), S. epidermidis, } \\
\text { B. subtilis, B. cereus, S. mutans, M. luteus }\end{array}$ & [204] \\
\hline B. deserti & Algeria & $\begin{array}{l}\text { AP: } \mathrm{CH}_{2} \mathrm{Cl}_{2}, \mathrm{MeOH} \text {; then isolat. } \\
\text { compds: } 40,223,227\end{array}$ & $\operatorname{MIC}(\mu M): 46-162$ & E. faecalis, P. aeruginosa, S. aureus & [90] \\
\hline B. inaequidens & Turkey & $\begin{array}{l}\text { AP: Ac; isolat. compds: 5, 25, } \\
79,103,105,107,108,109\end{array}$ & $\operatorname{MIC}(\mu \mathrm{g} / \mathrm{mL}): 25-50$ & S. aureus, B. subtilis, E. coli, P. aeruginosa & [76] \\
\hline B. nigra & Italy & S: EtOH & $\begin{array}{l}\text { Quantification of } \delta \text {-hemo- } \\
\text { lysin. Response in the prod- } \\
\text { uction of } \delta \text {-hemolysin, indi- } \\
\text { cating anti-QS activity in a } \\
\text { pathogenic MRSA isolate. } \\
\text { No inhibitory effect }\end{array}$ & S. aureus & [252] \\
\hline B. nigra & Pakistan & $\begin{array}{l}\text { S, L, R: EtOH then part. between } \\
\mathrm{W} \text { and } n-\mathrm{Hex}, \mathrm{EtOAc}, \mathrm{CHCl}_{3} \text {, } \\
\mathrm{BuOH}\end{array}$ & $A D$ at $5 \mathrm{mg} / \mathrm{mL}(\mathrm{mm}) 8-30$ & $\begin{array}{l}\text { E. coli, S. aureus, P. mirabilis, K. pneumoniae, E. faecalis, } \\
\text { S. typhi }\end{array}$ & [253] \\
\hline B. nigra & Serbia & S, L: EO & $\mathrm{MIC}(\mu \mathrm{g} / \mathrm{mL}): 2.5-5$ & $\begin{array}{l}\text { E. coli, S. aureus, B. mycoides, M. lysodeikticus, } \\
\text { B. subtilis, K. pneumoniae, C. albicans }\end{array}$ & [209] \\
\hline B. nigra & France & $\begin{array}{l}\text { shoots: } 50 \% \text { EtOH then isolat. } \\
\text { compds: } 220,223,227\end{array}$ & MIC $(\mu \mathrm{g} / \mathrm{mL}): 64-128$ & S. aureus, S. aureus MRSA, P. mirabilis & [187] \\
\hline B. nigra & Italy & WP: W & $\begin{array}{l}\text { MIC, dose-dependent bio- } \\
\text { film formation inhibition, } \\
\text { max inhib. at } 128 \mu \mathrm{g} / \mathrm{mL}\end{array}$ & S. aureus MRSA & [254] \\
\hline $\begin{array}{l}\text { B. nigra spp. } \\
\text { anatolica }\end{array}$ & Turkey & L: EtOH & $\operatorname{MIC}(\mu \mathrm{g} / \mathrm{mL}) 250-1000$ & $\begin{array}{l}\text { B. subtilis, B. cereus, S. aureus, E. coli, P. vulgaris, } \\
\text { S. typhimurium, P. aeruginosa }\end{array}$ & [255] \\
\hline $\begin{array}{l}\text { B. nigra spp. } \\
\text { anatolica }\end{array}$ & Turkey & L: EtOH & $\begin{array}{l}\text { AD at } 50 \mu \mathrm{g} / \mathrm{mL}(\mathrm{mm}) \\
10.0-19.2\end{array}$ & $\begin{array}{l}\text { B. cereus, P. aeruginosa, K. pneumoniae, S. capitis, } \\
\text { S. aureus, S. epidermidis, P. acnes, M. nonliquefaciens, }\end{array}$ & [256] \\
\hline $\begin{array}{l}\text { B. nigra ssp } \\
\text { foetida }\end{array}$ & Italy & EO & MIC, MBC (mg/mL): 3-7 & $\begin{array}{l}\text { E. coli, E. cloacae, P. aerouginosa, F. fluorescens, } \\
\text { S. aureus, S. epidermidis }\end{array}$ & [216] \\
\hline $\begin{array}{l}\text { B. pseudo- } \\
\text { dictamnus }\end{array}$ & Pakistan & $\begin{array}{l}\text { S, L, R: EtOH then part. bet. } \\
\text { W/n-Hex, EtOAc, } \mathrm{CHCl}_{3} \text {, BuOH }\end{array}$ & $\begin{array}{l}\mathrm{AD} \text { at } 2 \mu \mathrm{g} / \mathrm{mL}(\mathrm{mm}) \\
0.8-20\end{array}$ & $\begin{array}{l}\text { E. coli, S. aureus, P. mirabilis, K. pneumoniae, E. faecalis, } \\
\text { S. typhi }\end{array}$ & [257] \\
\hline $\begin{array}{l}\text { B. pseudo- } \\
\text { dictamnus }\end{array}$ & Greece & EO & $\begin{array}{l}\mathrm{MIC}(\mathrm{mg} / \mathrm{mL}) 0.45-10.15 \\
>20 \text { for } E . \text { coli }\end{array}$ & $\begin{array}{l}\text { S. aureus, S. epidermidis, E. coli, E. cloacae, } \\
\text { K. pneumoniae, P. aeruginosa }\end{array}$ & [220] \\
\hline B. rotundifolia & Turkey & $\begin{array}{l}\text { WP: MeOH then the extract } \\
\text { was split in W-soluble and } \\
\text { W-insoluble fractions }\end{array}$ & $\operatorname{MIC}(\mu \mathrm{g} / \mathrm{mL}):>72$ & $\begin{array}{l}\text { S. pneumoniae, B. cereus, A. Iwoffii, E. coli, } \\
\text { K. pneumoniae, C. perfringens }\end{array}$ & [238] \\
\hline B. saxatilis & Turkey & $\begin{array}{l}\text { F: Ac: then isolat. compds: } \\
5,18,25\end{array}$ & $\operatorname{MIC}(\mu \mathrm{g} / \mathrm{mL}): 25-50$ & $\begin{array}{l}\text { S. aureus, S. faecalis, E. coli, P. aeruginosa, } \\
\text { K. pneumaniae. }\end{array}$ & [80] \\
\hline $\begin{array}{l}\text { B. saxatilis ssp. } \\
\text { brachyodonta }\end{array}$ & Turkey & EO & $\operatorname{MIC}(\mu \mathrm{g} / \mathrm{mL}): 25-50$ & $\begin{array}{l}\text { E. coli, E. feacalis, B. subtilis, S. thyphimurium, S. aureus, } \\
\text { S. epidermidis, K. pneumoniae }\end{array}$ & [221] \\
\hline O. fruticosa & Egypt & EO & MIC $(\mu \mathrm{g} / \mathrm{mL}): 1.5-6$ & $\begin{array}{l}\text { B. subtilis, S. aureus, E. coli, S. epidermidis, S. faecalis, } \\
\text { K. aerogenes }\end{array}$ & [224] \\
\hline $\begin{array}{l}\text { O. fruticosa ssp. } \\
\text { schimperi }\end{array}$ & Yemen & EO & $\operatorname{MIC}(\mu \mathrm{g} / \mathrm{mL}): 310-1250$ & B. cereus, S. aureus, E. coli, P. aeruginosa & [222] \\
\hline O. integrifolia & Ethiopia & $\mathrm{L}: 80 \% \mathrm{MeOH}, \mathrm{CHCl}_{3}$ & $\operatorname{MIC}(\mathrm{mg} / \mathrm{mL}): 0.312$ & M. tuberculosis & $\begin{array}{r}{[258]} \\
\text { cont }\end{array}$ \\
\hline
\end{tabular}


$\checkmark$ Table 14 Continued

\begin{tabular}{|c|c|c|c|c|c|}
\hline Species & Origin & $\begin{array}{l}\text { Sample preparation } \\
\text { (plant part: solvent) }\end{array}$ & Test & Target & Ref \\
\hline O. integrifolia & Ethiopia & EO & $\mathrm{MIC}(\mathrm{mg} / \mathrm{mL})$ 5-100 & $\begin{array}{l}\text { E. coli 18/9, E. coli ATCC } 10536 \text {, E. coli CD/99/1, } \\
\text { E. coli K88, E. coli RP4, E. coli VC Sonawave } 3: 37 \text { C, } \\
\text { P. aeruginosa, S. boydii, S. dysentery, S. flexneri, } \\
\text { S. soneii } 1 \text {, S. soneii BCH 217, V. cholera, B. subtilis }\end{array}$ & [56] \\
\hline O. limbata & Pakistan & $\begin{array}{l}\text { WP: } 70 \% \text { EtOH. then ext. } \\
\text { solubilized in DMSO, EtOH, } \\
\text { MeOH }\end{array}$ & MIC (mg/mL): 0.2-5 & $\begin{array}{l}\text { B. subtilis, L. monocytogenes, S. aureus, E. coli, } \\
\text { P. aeruginosa, Salmonella spp. }\end{array}$ & [259] \\
\hline O. persica & Iran & AP: $\mathrm{MeOH}$ & $\mathrm{MIC}(\mathrm{mg} / \mathrm{mL}): 0.5-2$ & $\begin{array}{l}\text { E. coli, B. subtilis, S. aureus, S. epidermis, P. aeruginosa, } \\
\text { S. typhi, K. pneumonia, A. niger }\end{array}$ & [260] \\
\hline O. persica & Iran & $\begin{array}{l}\text { AP: } 70 \% \text { EtOH, } n \text { - } \mathrm{Hex}, \mathrm{CHCl}_{3} \text {, } \\
\mathrm{MeOH}\end{array}$ & MIC (mg/mL): 1.25-25 & $\begin{array}{l}\text { E. coli, L. monocytogen, E. faecalis, S. aureus, } \\
\text { S. epidermidis, B. subtiltis, P. aeruginosa, } \\
\text { Salmonella spp. Klebsiella spp. }\end{array}$ & [261] \\
\hline
\end{tabular}

The Ethiopian plant $O$. integrifolia, in particular the leaf extract in $80 \% \mathrm{MeOH}$, was also effective in contrasting diabetes in mice and rats [293]. STZ induced diabetic mice receiving doses from 100 to $400 \mathrm{mg} / \mathrm{kg}$ b.w. of extract displayed time-dependent hypoglycemic effects. After $4 \mathrm{~h}$, the dose of $200 \mathrm{mg}$ reduced the hematic glucose from 375 to $159 \mathrm{mg} / \mathrm{dL}$.

Three extracts were obtained from the AP of $B$. cinerea (India) by brewing them in $\mathrm{PE}$, EtOAc, and $\mathrm{MeOH}$ [294]. They were active in reducing blood glucose levels in diabetic rats both with acute (after $4 \mathrm{~h}$ ) and with chronic (after $21 \mathrm{~d}$ ) alloxana-induced disease. Total reduction of glucose reached values (36-42\%) comparable to the standard antidiabetic drug glibenclamide. Other authors [267] reported slightly different values for the activity of the same extracts, together with the EtOH extract, assessed in the reduction of hematic glucose in STZ-induced diabetic rats: from 18.2 to $23.4 \%$ reduction after $24 \mathrm{~h}$ at $100 \mathrm{mg} / \mathrm{kg} \mathrm{b.w.} \mathrm{Furthermore,}$ similar results in reducing murine glycemia were obtained with the butanol and water extract of the same taxon [233]. In the last study, the lipidic profile, the hepatic glycogen content and the pancreatic parameters (SOD, GSH, and PGx) were also evaluated after $15 \mathrm{~d}$ of extract treatments at $50 \mathrm{mg} / \mathrm{kg} \mathrm{b.w.} \mathrm{Almost} \mathrm{all} \mathrm{of}$ the parameters were normalized as compared to standard values, and these beneficial effects were confirmed by the histopathological evaluation of pancreatic and hepatic tissue dissections. Take note that the dose of extract implemented in this study is significantly lower than the average doses normally utilized in similar contexts.

4-Methoxybenzo[b]azet-2(1H)-one (214) and 3 $\beta$-hydroxy-35(cyclohexyl-5'-propan-7'-one)-33-ethyl-34-methyl-bacteriohop16-ene (178), isolated from the aerial part of $B$. cinerea (India) [182], significantly reduced the blood glucose level in alloxan-induced diabetic rats at the dose of $10 \mathrm{mg} / \mathrm{kg}$ b.w. administered orally.

An interesting protective effect for hyperlipidemia was investigated for the aqueous extract of $B$. arabica (syn. L. urticifolia) in a Triton WR-1339 induced hyperlipidemic rat model [295]. The administration of a $100-400 \mathrm{mg} / \mathrm{kg}$ b. w. dose for $24 \mathrm{~h}$ was able to restore normal values of plasma lipidic parameters, total cholesterol, TG, LDL, VLDL, and to significantly raise the HDL level.

\section{Other bioactivities in vivo}

The fruit extract of $B$. undulata obtained in $\mathrm{EtOH} /$ water 7:3 was shown to be effective as a fertility controller in Albino rats [296]. The effects were time dependent: after a treatment of $4 \mathrm{wk}$ at $15 \mathrm{mg} / \mathrm{kg}$ b.w. per day, the numbers of pregnancies was not reduced significantly, while only a slight reduction in embryo and ovarian weights was observed. On the contrary, when the treatment was prolonged until $12 \mathrm{wk}$, the percentage of embryo implantation and pregnancies with respect to the controls was statistically relevant.

The extract of $O$. persica AP in $\mathrm{MeOH}$ was tested for its healing promoting activity in the skin of Wistar rats [297]. The healing process of burns provoked in the dorsal part of animals was accelerated by the application of a ointment in which the extract was dispersed. The histological evaluation evidenced an increase in fibroblast proliferation, angiogenesis and re-epithelialization that improved in a 5- to 14-d time range.

Ischemia-reperfusion is a dangerous syndrome that can cause severe injuries to remote organs, due to multiple effects, including the increase in reactive oxygenated radicals and general inflammation conditions. The ethanolic extract of O. persica (Iran) demonstrated protective effects toward renal injury in rats suffering of a hindlimb ischemia reperfusion surgically induced by clamping the femoral artery [298]. Reperfusion induced kidney damage including the increase of water uptake, creatinine excretion rate, and kidney/body weight. The animals treated with $300 \mathrm{mg} / \mathrm{kg}$ b. w. of extract $2 \mathrm{~d}$ before intervention had the abovementioned renal functionality parameters at levels comparable with the negative control.

The antihypertensive effect of the AP extract $(70 \% \mathrm{EtOH})$ of $O$. persica was proven in Wistar rats suffering from dexamethasone-induced hypertension [299]. The systolic pressure increase from 115 to $143 \mathrm{mmHg}$ was completely suppressed by a dose of $400 \mathrm{mg} / \mathrm{kg}$ b. w. per day of the extract, administered $2 \mathrm{~d}$ before starting the dexamethasone treatment. The hematic $\mathrm{H}_{2} \mathrm{O}_{2}$ and FRAP values increased by dexamethasone were also normalized in the animals receiving the plant brew. 
- Table 15 Antifungal activity of Ballota and Otostegia taxa.

\begin{tabular}{|c|c|c|c|c|c|}
\hline Species & Origin & $\begin{array}{l}\text { Sample preparation } \\
\text { (plant part: solvent) }\end{array}$ & Test & Target & Ref. \\
\hline B. acetabulosa & Turkey & L, R: $50 \%$ EtOH & $\operatorname{MIC}(\mathrm{mg} / \mathrm{mL}) 1.56-25.0$ & $\begin{array}{l}\text { C. albicans, C. tropicalis, C. guilliermondii, } \\
\text { Cryptococcus neoformans, C. laurentii }\end{array}$ & [263] \\
\hline B. deserti & Algeria & $\begin{array}{l}\mathrm{EO} \\
\mathrm{L}: \mathrm{MeOH}\end{array}$ & $\begin{array}{l}\text { MIC biofilm formation EO } \\
(\mu \mathrm{L} / \mathrm{mL}) 25 ; \mathrm{MeOH} \text { extr, } \\
(\mathrm{mg} / \mathrm{mL}) 12.5\end{array}$ & C. albicans & [204] \\
\hline B. inaequidens & Turkey & $\begin{array}{l}\text { AP: Ac; then isolat. } \\
\text { compds: } 5,25,79, \\
103,105,107,108 \text {, } \\
109\end{array}$ & $\mathrm{MIC}(\mu \mathrm{g} / \mathrm{mL}) 3.1-12.5$ & C. albicans, C. crusei & [76] \\
\hline B. nigra & Paikstan & $\begin{array}{l}\mathrm{S}, \mathrm{L}, \mathrm{R}: \text { EtOH then } \\
\text { partition between } \\
\mathrm{W} / \mathrm{n}-\mathrm{Hex}, \mathrm{EtOAc} \text {, } \\
\mathrm{CHCl}_{3}, \mathrm{BuOH}\end{array}$ & $\begin{array}{l}\text { Agar tube diluition, results } \\
\text { reported as inhibition } \\
\text { "positive" or "negative"; } \\
\text { crude extrat always posi- } \\
\text { tive at } 2 \mathrm{mg} / \mathrm{mL}\end{array}$ & A. niger, A. flavus, A. fumigatus, F. solani & [253] \\
\hline B. nigra ssp. anatolica & Turkey & L: EtOH & $\operatorname{MIC}(\mu \mathrm{g} / \mathrm{mL}): 500-1000$ & C. albicans, D. hansenii, K. fragilis, R. rubra & [255] \\
\hline B. nigra ssp foetida & Italy & EO & $\begin{array}{l}\mathrm{MIC}(\mathrm{mg} / \mathrm{mL}) 5.5 \\
\mathrm{MBC}(\mathrm{mg} / \mathrm{mL}) 15.0\end{array}$ & C. albicans, C. glabrata, C. tropicalis & [216] \\
\hline B. pseudodictamnus & Pakistan & $\begin{array}{l}\text { S, L, R: EtOH then } \\
\text { partition between } \\
W / n \text {-Hex, EtOAc, } \\
\mathrm{CHCl}_{3}, \mathrm{BuOH}\end{array}$ & $\begin{array}{l}\text { Agar tube dilution, results } \\
\text { reported as inhibition } \\
\text { "positive" or "negative" at } \\
2 \mathrm{mg} / \mathrm{mL}\end{array}$ & A. niger; A. fumigates, A. flavus; F. solani & [257] \\
\hline B. pseudodictamnus & Greece & EO & Not active & C. albicans, C. tropicalis, C. glabrata & [220] \\
\hline B. rotundifolia & Turkey & $\begin{array}{l}\text { WP: } \mathrm{MeOH} \text { then the } \\
\text { extract was split in } \\
\text { W-soluble and } \\
\text { W-insoluble fractions }\end{array}$ & $\operatorname{MIC}(\mu \mathrm{g} / \mathrm{mL})>72$ & C. albicans, C. krusei & [238] \\
\hline B. saxatilis & Turkey & $\begin{array}{l}\text { L: Ac then isolat. } \\
\text { compds: } 5,18,25\end{array}$ & $\begin{array}{l}\text { Agar diluititon MIC } \\
(\mu \mathrm{g} / \mathrm{mL}): 1.5-3.1\end{array}$ & C. albicans & [80] \\
\hline $\begin{array}{l}\text { B. saxatilis ssp. } \\
\text { brachyodonta }\end{array}$ & Turkey & EO & MIC ( $\mu \mathrm{g} / \mathrm{mL}): 25$ & C. albicans (clinic strain), C. parapsilosis & [221] \\
\hline B. undulata & Egypt & $\begin{array}{l}\text { L, S, F: EtOH, EtOAc, } \\
\mathrm{CHCl}_{3}, n-\mathrm{Hex}\end{array}$ & $\operatorname{MIC}(\mathrm{mg} / \mathrm{mL}): 25$ - > 150 & $\begin{array}{l}\text { T. rubrum, T. tonsurans, C. albicans, } \\
\text { C. tropicum, P. lilacinus, P. variotii, } \\
\text { S. bervicaulis }\end{array}$ & [264] \\
\hline O. integrifolia & Ethiopia & EO & MIC $(\mu \mathrm{g} / \mathrm{mL}): 50-100$ & $\begin{array}{l}\text { A. niger, C. albicans, P. funiculosum, } \\
\text { P. notatum }\end{array}$ & [56] \\
\hline O. fruticosa & Egypt & EO & $\operatorname{MIC}(\mu \mathrm{g} / \mathrm{mL}): 6.0,16.0$ & C. albicans, S. cerevisiae & [224] \\
\hline O. limbata & Paikstan & $\begin{array}{l}\text { WP: } \mathrm{MeOH} \text {, then } \\
\text { partition in } n-\mathrm{Hex} \text {, } \\
\mathrm{CHCl}_{3} \text {, EtOAc, BuOH }\end{array}$ & MIC $(\mathrm{mg} / \mathrm{mL}) 0.18-1.5$ & S. setubal, P. pickettii, S. aureus, M. luteus & [265] \\
\hline O. persica & Iran & AP: $\mathrm{MeOH}$ & MIC (mg/mL): 1.0 & C. albicans & {$[260]$} \\
\hline
\end{tabular}

\section{Anti-malarial activity}

A significant antimalarial effect in Plasmodium berghei Vinke \& Lips-infected mice was disclosed for 0 . integrifolia collected in Ethiopia [300]. Mice were administrated with 200, 400, and $800 \mathrm{mg} / \mathrm{kg}$ b.w. doses of the leaf extract prepared in chloroform, $\mathrm{MeOH}$, and water. The survival of the animals treated with polar extract was dose-dependent and significantly higher than that of the negative control ( 10.5 vs. $7.5 \mathrm{~d}$ of $\mathrm{H}_{2} \mathrm{O}$ ext. at max. dose; 13.5 vs. 7 for $\mathrm{MeOH}$ ext.). General lack of toxicity was proven until reaching a $2000 \mathrm{mg} / \mathrm{kg}$ b. w. dose. Also in this case, the very high dose of extract employed may be an obstacle for possible developments toward application to humans. However, a study appear- ing in the literature in the same year [101] resulted in the bioguided isolation of the labdane diterpene otostegindiol (43) from the methanolic extract of $O$. integrifolia leaves. This compound showed chemosuppressive properties against $P$. berghei with a maximum suppression ratio of $73.16 \%$ at $100 \mathrm{mg} / \mathrm{kg}$. Additionally, the EtOH extract of the aerial part of Iranian O. persica [301] demonstrated antimalarial activity in $P$. berghei infected mice with $\mathrm{ED}_{50}$ of $45 \mathrm{mg} / \mathrm{kg}$ b. w. Furthermore, an interesting synergistic effect was observed when CQ-sensitive animals were treated with a combination of this drug and the plant extract; for example, a combination of $70 \%$ of the $E D_{50}$ of CQ with $30 \%$ of extract caused a $26 \%$ increase in the mean effective dose. 
The effective antiprotozoal activity against another relevant malarial parasite, Plasmodium falciparum Welch, was disclosed in both the PE and the chloroform extracts of $B$. cinerea from India [302]. The $\mathrm{IC}_{50}$ values for the 2 materials were 4.39 and $1.84 \mu \mathrm{g} /$ $\mathrm{mL}$, respectively.

\section{Hepatoprotective effects}

The AP extract $(80 \% \mathrm{MeOH})$ of 0 . persica collected in Iran [303] was effective in strongly reducing liver damage in $\mathrm{CCl}_{4}$ intoxicated rats $(2.5 \mathrm{mg} / \mathrm{kg}$ b. w.). After administrating $400 \mathrm{mg} / \mathrm{kg}$ b. w. of extract, hematic liver damage parameters were significantly ameliorated with respect to untreated animals: ALT 13\%, AST 11.6\%, plasma MDA 6.7\%, liver MDA $11.4 \%$, liver GSH + 21\%. Histological evaluation of liver sections demonstrated the prevention of tissue degradation in treated rats. A similar protective effect was described for the aqueous extract of the AP of B. glandulosissima from Turkey [245]. Liver damage indicators in the blood of $\mathrm{CCl}_{4}$ treated rats $(0.8 \mathrm{~mL} / \mathrm{kg}$ b.w.) were reduced significantly with $100 \mathrm{mg} / \mathrm{kg}$ b. w. of extract: AST 59\%, ALT 47\%, ALP 43\%, bilirubin $47 \%$. The efficacies of the 2 above-cited treatments are hardly comparable considering the differences in applied dose intoxication, in the extraction procedure and in the feeding procedures of the animals involved in these studies. Similar protective effects on the liver were found in the AP extract $(70 \% \mathrm{MeOH})$ of 0 . persica collected in Iran [304] administrated to rats at $80-120 \mathrm{mg} / \mathrm{kg}$ b. w.

The hepatoprotective effect of $B$. cinerea (syn. Roylea elegans Wall. ex Benth.) collected in India was assessed for the AP extract obtained in $\mathrm{EtOH} / \mathrm{H}_{2} \mathrm{O} 1: 1$ in a $\mathrm{CCl}_{4}$ and paracetamol toxicity induced model in rats [305]. Hepatic damage was evaluated by several blood parameters, such as SGOT, SGPT, ALP, and TB, after treatment with $100-400 \mathrm{mg} / \mathrm{kg}$ b. w. of extract for $7 \mathrm{~d}$. Also, the liver oxidative stress indicators GSH and TBARS were followed. The pathological displacement of all of the indicators was reduced in a dose-dependent fashion and complete normalization occurred at the dose of $400 \mathrm{mg}$.

\section{Enzymatic activity modulation}

There are a number of investigations aimed at evaluating the plants from Ballota and Otostegia genera as a source of useful bioactive compounds isolated from the complex blend of their secondary metabolites. The extract of $\mathrm{O}$. limbata root in $\mathrm{MeOH}$ led to the isolation and identification of 3 new tricyclic cis-clerodane diterpenes: limbatolide A (64), limbatolide B (65), and limbatolide $C(66)$ that were assessed of their inhibitory potential against acetylcholinesterase (AChE; EC 3.1.1.7) and butyrylcholinesterase (BChE; EC 3.1.1.8). The inhibition activity was higher for the latter enzyme ( $\mathrm{IC}_{50} 22.3,17.5$ and $14.2 \mu \mathrm{M}$, respectively) than for the former ( $\mathrm{IC}_{50} 38.5,47.2$, and $\left.103.7 \mu \mathrm{M}\right)$ [112]. These enzymatic systems were also the targets of another similar work concerning the isolation of 6 clerodane tricyclic diterpenes from the chloroform extract of $B$. limbata [107]: ballatenolide A (59), 15-methoxypatagonic acid (71), patagonic acid (72), and limbatenolides A-C (54-56). All of the compounds showed inhibitory activity with BChE with $\mathrm{IC}_{50}$ values ranging from 24.9 to $51.0 \mu \mathrm{M}$, lower than the standard inhibitor galanthamine $(8.5 \mu \mathrm{M})$. The first group of 3 compounds also showed a moderate inhibitory activity with
AChE with $\mathrm{IC}_{50}$ values between 50.0 and $102 \mathrm{mM}$ (galanthamine $0.50 \mu \mathrm{M})$. The inhibition activity toward the 2 enzymatic systems was also assessed with the $\mathrm{MeOH}$ extract of the aerial part of $B$. deserti from Algeria [204], obtaining moderate $I_{50}$ values: $277.4 \mu \mathrm{g} / \mathrm{mL}$ for AChE and $93.3 \mu \mathrm{g} / \mathrm{mL}$ for BChE.

Ballotenic acid (60) and ballodiolic acid (62) were isolated from the chloroform soluble fraction of the $\mathrm{MeOH}$ extract of $B$. limbata [110] and displayed inhibitory potential against lipoxygenase enzyme in a concentration-dependent fashion with $\mathrm{IC}_{50}$ values of 99.6 $\mu \mathrm{M}$ and $38.3 \mu \mathrm{M}$, respectively.

Furthermore, the crude $n$-Hex extract of $B$. nigra subsp. kurdica [306] from Iran was investigated for its possible tyrosinase inhibitory activity by the colorimetric Tyrosinase inhibition assay $\left(\mathrm{IC}_{50}=3.67 \mu \mathrm{g} / \mathrm{mL}\right)$; however, no attempt was made to isolate individual active molecules.

Tyrosinase was also effectively inhibited by 2 compounds isolated from the AP extract of $B$. cinerea from India [182]: 4methoxybenzo[b]azet-2(1H)-one (214) and 3 $\beta$-hydroxy-35-(cyclohexyl-5'-propan-7'-one)-33-ethyl-34-methyl-bacteriohop-16ene (178) with inhibition rate of 83.0 and $58.2 \%$, respectively, at $100 \mu \mathrm{M}$. These compounds were also effective inhibitors of $\alpha$-glucosidase (78.5\% and $58.4 \%)$. This inhibitory activity is related to the above discussed antidiabetic activity in vivo of these compounds. The $\alpha$-glucosidase and $\beta$-glucosidase reduction activities were evaluated in a study on the antidiabetic activity in vitro and in vivo of some extracts of $B$. cinerea from India [294]. Three fractions were found more active, respectively, obtained with PE, EtOAc, and $\mathrm{MeOH}$; their activity reduction power ranged about from 55 to $80 \%$, with the $\mathrm{MeOH}$ extract being the most active. In another work [267], these extracts were tested in an in vitro inhibitory activity test against protein tyrosine phosphatase-1B, showing results ranging from 39 to $65 \%$ inhibition at $100 \mu \mathrm{M}$.

$\alpha$-Amylase, an enzyme involved in saccharide metabolism which is believed to possess preventive properties for type II diabetes, is strongly inhibited by polyphenolic compounds [307]. For this reason, the inhibitory activity of the extracts of $O$. persica was evaluated in association with the antioxidant activity (DPPH test, see $>$ Table 13) [243]. The initial crude extract obtained in EtOH was then partitioned in solvents of different polarities: PE, EtOAc, $\mathrm{CHCl}_{3}, n-\mathrm{BuOH}, \mathrm{EtOH}$. The enzymatic parameters were measured for all of the fractions: inhibition rate from 53.3 (pet. Ether) to $99.4 \%$ (EtOAc). The author attempted to relate the total phenolic content of the extracts both with the antioxidant and the enzyme inhibition activity.

The 2 new flavonoidal glucosides leufolins A (163) and B (152) isolated from $B$. arabica (syn. L. urticifolia) have shown to be potent inhibitors of $B C h E$ enzyme ( $\mathrm{IC}_{50}$ values 1.6 and $3.6 \mu \mathrm{M}$, respectively) when compared to serine, used as a positive control $\left(\mathrm{IC}_{50}\right.$ $0.93 \mu \mathrm{M})$. On the other hand, very weak activity was observed against acetylcholinestrase ( $\mathrm{IC}_{50}$ values 74.5 and $72.3 \mu \mathrm{M}$, respectively), compared to eserine $\left(\mathrm{IC}_{50}=0.04 \mu \mathrm{M}\right)$ [171]. The new steroid leucisterol (179), also isolated from the same species, showed potent inhibitory activity against butyrylcholinesterase enzyme $\left(\mathrm{IC}_{50}=3.2 \mu \mathrm{M}\right)$. [181]. 


\section{Conclusions}

In this review a complete recognition of the volatile and not volatile secondary metabolites occurring in the Ballota and Otostegia genera has been carried out. The ${ }^{13} \mathrm{C}$ NMR data of diterpenes reported in literature have been collected for comparison purposes in structural determination. Some relevant studies on several biological activities have been reported that include antioxidant, anti-inflammatory, antibacterial, antifungal, antitumor, and antidiabetic.

\section{Acknowledgements}

This work was supported by grant from MIUR-ITALY PRIN 2015 (Project N. 2015MSCKCE_003) and PRIN 2017 (Project N. 2017A95NC]).

\section{Conflict of Interest}

The authors declare that they have no conflict of interest.

\section{References}

[1] Erdtman G. Pollen morphology and plant taxonomy. IV. Labiatae, Verbenaceae and Avicennaceae. Svensk Bot Tidskr 1945; 39: 279-285

[2] Cantino PD, Sanders RW. Subfamilial classification of Labiatae. Syst Bot 1986; 11: 163-185

[3] Patzäk A. Revision der Gattung Ballota Section Âcanthoprasium und Section Beringeria. Ann Naturhist Mus Wien 1958; 62: 57-86

[4] Patzäk A. Revision der Gattung Ballota Section Âcanthoprasium und Section Beringeria. Ann Naturhist Mus Wien 1959; 63: 33-81

[5] Patzäk A. Revision der Gattung Ballota Section Âcanthoprasium und Section Beringeria. Ann Naturhist Mus Wien 1961; 64: 42-56

[6] The Plant List. Available at www.theplantlist.org. Accessed September 5 , 2018

[7] Bendiksby M, Thorbek L, Scheen AC, Lindqvist C, Ryding O. An updated phylogeny and classification of Lamiaceae subfamily Lamioideae. Taxon 2011; 60: 471-484

[8] Harley RM, Atkins S, Budantsev AL, Cantino PD, Conn BJ, Grayer R, Harley MM, De Kok R, Krestovskaja T, Morales R, Paton AJ, Ryding O, Upson T. Labiatae. In: Kadereit JW, ed. The Families and Genera of vascular Plants. Vol. 7: Lamiales. Berlin, Heidelberg: Springer; 2004: 167-275

[9] Hedge IC. A global Survey of the Biogeography of the Labiatae. In: Harley RM, Reynolds T, eds. Advances in labiate Science. Kew: The Royal Botanic Gardens; 1992: 7-17

[10] Scheen AC, Albert VA. Nomenclatural and taxonomic changes within the Leucas clade (Lamioideae; Lamiaceae). Syst Geogr PI 2007; 77: 229-238

[11] Tuzlaci E, Sadikoğlu E. Turkish folk medicinal plants, part VI: Koçarli (Aydin). J Fac Pharm Istanbul 2007; 39: 25-37

[12] Dumlu MU, Bulut GE. Some biological activity investigations on Ballota acetabulosa. J Fac Pharm Istanbul 2007; 39: 53-56

[13] Nortje JM, Van Wyk BE. Medicinal plants of the Kamiesberg, Namaqualand, South Africa. J Ethnopharmacol 2015; 171: 205-222

[14] Scott G, Springfield EP, Coldrey N. A pharmacognostical study of 26 South African plant species used as traditional medicines. Pharm Biol 2004; 42: 186-213

[15] Cock IE, van Vuuren SF. Anti-proteus activity of some South African medicinal plants: their potential for the prevention of rheumatoid arthritis. Inflammopharmacology 2014; 22: 23-36
[16] Akhtar K, Khan SB, Malik A. Phytochemistry and pharmacological aspects of Leucas urticifolia (Vahl) Benth. J Pharm Res Health Care 2010; 2: 197-203

[17] Sadeghi Z, Kuhestani K, Abdollahi V, Mahmood A. Ethnopharmacological studies of indigenous medicinal plants of Saravan region, Baluchistan, Iran. J Ethnopharmacol 2014; 153: 111-118

[18] Bano A, Ahmad M, Zafar M, Sultana S, Rashid S, Khan MA. Ethnomedicinal knowledge of the most commonly used plants from Deosai Plateau, Western Himalayas, Gilgit Baltistan, Pakistan. J Ethnopharmacol 2014; 155: 1046-1052

[19] Natarajan B, Paulsen BS, Korneliussen V. An ethnopharmacological study from Kulu district, Himachal Pradesh, India: traditional knowledge compared with modern biological science. Pharm Biol 2000; 38: 129-138

[20] Rawat R, Vashistha DP. Roylea cinerea (D. Don) Baillon: a traditional curative of diabetes, its cultivation prospects in Srinagar Valley of Uttarakhand. Int J Adv Pharm Biol Chem 2013; 2: 372-375

[21] Dangwal LR. Ethnomedicinal plants used for the treatment of diabetes among the villagers of Narendra Nagar block, district Tehri Garhwal, Uttarakhand, India. World J Pharm Pharm Sci 2015; 4: 1178-1184

[22] Bisht S, Pangti J, Pundir S, Das A, Devi J. Total phenolic and total flavonoid compounds in the leaves of Roylea cinerea using hydroalcoholic extract. World J Pharm Pharm Sci 2016; 7: 1753-1759

[23] Hammiche V, Maiza K. Traditional medicine in Central Sahara: pharmacopoeia of Tassili N'ajjer. J Ethnopharmacol 2006; 105: 358-367

[24] Dendougui H, Seghir S, Belloum Z, Benayache F, Leon F, Brouard I, Bermejo J, Benayache S. A new labdane diterpene and other constituents from Marrubium deserti Noe ex Coss. Rec Nat Prod 2011; 5: 300-304

[25] Edziri H, Mastouri M, Aouni M, Verschaeve L. Polyphenols content, antioxidant and antiviral activities of leaf extracts of Marrubium deserti growing in Tunisia. S Afr J Bot 2012; 80: 104-109

[26] Teixidor-Toneu I, Martin G], Ouhammou A, Puri RK, Hawkins JA. An ethnomedicinal survey of a Tashelhit-speaking community in the High Atlas, Morocco. J Ethnopharmacol 2016; 188: 96-110

[27] Kechar K, Hellal B, Ayad N, Benahmed-Djilali A. Ethnobotanical investigation of Ballota hirsuta (Benth) at Sidi Bel Abbes (Algeria). Phytotherapie 2016; 14: 343-348

[28] Zheng YF, Yin W, Lin LL, Du SS, Shan T, Wang Y. Advances in studies on Panzeria alaschanica. Zhong Cao Yao 2007; 38: 1434-1436

[29] Olennikov DN, Tankhaeva LM. Chemical study of Panzerina lanata. Chem Nat Compd 2011; 47: 802-804

[30] Rexhepi B, Mustafa B, Hajdari A, Rushidi-Rexhepi ], Quave CL, Pieroni A. Traditional medicinal plant knowledge among Albanians, Macedonians and Gorani in the Sharr Mountains (Republic of Macedonia). Genet Resour Crop Evol 2014; 60: 2055-2080

[31] Ciocarlan N. Family Lamiaceae: main important spontaneous medicina and aromatic species in the Republic of Moldova. J Bot 2016; 8: 86-91

[32] Šarić-Kundalić B, Dobeš C, Klatte-Asselmeyer V, Saukel J. Ethnobotanical survey of traditionally used plants in human therapy of east, north and north-east Bosnia and Herzegovina. J Ethnopharmacol 2011; 133: 1051-1076

[33] Pieroni A. Medicinal plants and food medicines in the folk traditions of the upper Lucca Province, Italy. J Ethnopharmacol 2000; 70: 235-273

[34] Mulas M. Traditional uses of Labiatae in the Mediterranean area. Acta Hortic 2006; 723: 25-32

[35] Pieroni A, Quave C, Nebel S, Heinrich M. Ethnopharmacy of the ethnic Albanians (Arbëreshë) of northern Basilicata, Italy. Fitoterapia 2001; 73: 217-241

[36] Pieroni A, Quave CL, Santoro RF. Folk pharmaceutical knowledge in the territory of the Dolomiti Lucane, inland southern Italy. J Ethnopharmacol 2004; 95: 373-384 
[37] Redzić SS. The ecological aspect of ethnobotany and ethnopharmacology of population in Bosnia and Herzegovina. Coll Antropol 2007; 31: 869-890

[38] Nićiforović N, Mihailović V, Mašković P, Solujić S, Stojković A, Muratspahić DP. Antioxidant activity of selected plant species; potential new sources of natural antioxidants. Food Chem Toxicol 2010; 48: 31253130

[39] Gonzalez JA, Garcia-Barriuso M, Gordaliza M, Amich F. Traditional plantbased remedies to control insect vectors of disease in the Arribes del Duero (Western Spain): an ethnobotanical study. J Ethnopharmacol 2011; 138: 595-601

[40] Yeflilada E, Honda G, Sezik E, Tabata M, Goto T, Ikeshiro Y. Traditional medicine in Turkey IV. Folk medicine in the Mediterranean subdivision. J Ethnopharmacol 1993; 39: 31-38

[41] Yeflilada E, Honda G, Sezik E, Tabata M, Fujita T, Tanaka T, Takeda Y, Takaishi Y. Traditional medicine in Turkey V. Folk medicine in the inner Taurus Mountains. J Ethnopharmacol 1995; 46: 133-152

[42] Tuzlaci E, Aymaz PE. Turkish folk medicinal plants, part IV: Gönen (Balikesir). Fitoterapia 2001; 72: 323-343

[43] Kültür S. Medicinal plants used in Kirklareli Province (Turkey). J Ethnopharmacol 2007; 111: 341-364

[44] Mothana RAA, Kriegisch S, Harms M, Wende K, Lindequist U. Assessment of selected Yemeni medicinal plants for their in vitro antimicrobial, anticancer, and antioxidant activities. Pharm Biol 2011; 49: 200-210

[45] Mothana RA, Al-Musayeib NM, Al-Ajmi MF, Cos P, Maes L. Evaluation of the in vitro antiplasmodial, antileishmanial, and antitrypanosomal activity of medicinal plants used in Saudi and Yemeni traditional medicine. Evid Based Complement Alternat Med 2014; 905639: 1-7

[46] Meragiaw M, Asfaw Z, Argaw M. The status of ethnobotanical knowledge of medicinal plants and the impacts of resettlement in Delanta, Northwestern Wello, Northern Ethiopia. Evid Based Complement Alternat Med 2016; 2016: 5060247

[47] Kidane D, Tomass Z, Dejene T. Community knowledge of traditional mosquito repellent plants in Kolla Temben District, Tigray, Northern Ethiopia. Sci Res Essays 2013; 8: 1139-1144

[48] Seyoum G, Zerihun G. An ethnobotanical study of medicinal plants in Debre Libanos Wereda, Central Ethiopia. Afr J Plant Sci 2014; 8: 366-379

[49] Rahman MA, Mossa JS, Al-Said MS, Al-Yahya MA. Medicinal plant diversity in the flora of Saudi Arabia 1: a report on seven plant families. Fitoterapia 2004; 75: 149-161

[50] Waka M, Hopkins R], Curtis C. Ethnobotanical survey and testing of plants traditionally used against hematophagous insects in Eritrea. J Ethnopharmacol 2004; 95: 95-101

[51] Teklehaymanot T, Giday M, Medhin G, Mekonnen Y. Knowledge and use of medicinal plants by people around Debre Libanos monastery in Ethiopia. J Ethnopharmacol 2007; 111: 271-283

[52] Karunamoorthi K, Husen E. Knowledge and self-reported practice of the local inhabitants on traditional insect repellent plants in Western Hararghe zone, Ethiopia. J Ethnopharmacol 2012; 141: 212-219

[53] Giday G, Teklehaymanot T, Animut A, Mekonnen Y. Medicinal plants of the Shinasha, Agew-awi and Amhara peoples in northwest Ethiopia. J Ethnopharmacol 2007; 110: 516-525

[54] Mekuria AB, Belachew SA, Tegegn HG, Ali DS, Netere AK, Lemlemu E, Erku DA. Prevalence and correlates of herbal medicine use among type 2 diabetic patients in Teaching Hospital in Ethiopia: a cross-sectional study. BMC Complement Altern Med 2018; 18: 85

[55] Tesfaye H, Hishe M, Badasa S. Phytochemical and ethno botanical study of medicinal plants used to treat ectoparasites in ruminant animals in eastern Tigray, northern Ethiopia. Int J Pharmacogn (Panchkula, India) 2015; 2: 466-472

[56] Tadesse S, Messele B, Seyoum A, Mazumder A, Bucar F, Asres K. Essential oil of Otostegia integrifolia Benth: composition, antimicrobial and antioxidant activities. Ethiop Pharm J 2011; 29: 79-86
[57] Chopra RN, Nayar SL, Chopra IC. Glossary of Indian medicinal Plant. India: Indian Council of Scientific \& Industrial Research; 1956: 183

[58] Adnan M, Hussain J, Shah MT, Shinwari ZK, Ullah F, Bahader A, Khan N, Khan AL, Watanabe T. Proximate and nutrient composition of medicinal plants of humid and sub-humid regions in north-west Pakistan. J Med Plants Res 2010; 4: 339-345

[59] Abbasi AM, Khan MA, Ahmad M, Zafar M, Jahan S, Sultana S. Ethnopharmacological application of medicinal plants to cure skin diseases and in folk cosmetics among the tribal communities of North-West Frontier Province, Pakistan. J Ethnopharmacol 2010; 128: 322-335

[60] Haq F, Ahmad H, Alam M. Traditional uses of medicinal plants of Nandiar Khuwarr catchment (District Battagram), Pakistan. J Med Plants Res 2011; 5: 39-48

[61] Rehman K, Mashwani ZUR, Khan MA, Ullah Z, Chaudhary HJ. An ethno botanical perspective of traditional medicinal plants from the Khattak tribe of Chonthra Karak, Pakistan. J Ethnopharmacol 2015; 165: 251259

[62] Murad W, Ahmad A, Gilani SA, Khan MA. Indigenous knowledge and folk use of medicinal plants by the tribal communities of Hazar Nao forest, Malakand district, North Pakistan. J Med Plants Res 2011; 5: 1072-1086

[63] Iqbal H, Sher Z, Khan ZU. Medicinal plants from salt range Pind Dadan Khan, district Jhelum, Punjab, Pakistan. J Med Plants Res 2011; 5: $2157-$ 2168

[64] Adnan M, Bibi R, Azizullah A, Andaleeb R, Mussarat S, Tariq A, Ullah R, Elsalam NM, Khan AL, Begum S. Ethnomedicinal plants used against common digestive problems. Afr J Tradit Complement Altern Med 2015; 12: 99-117

[65] Rashid S, Ahmad M, Zafar M, Sultana S, Ayub M, Khan MA, Yaseen G. Ethnobotanical survey of medicinally important shrubs and trees of Himalayan region of Azad Jammu and Kashmir, Pakistan. J Ethnopharmacol 2015; 166: 340-351

[66] Ahmad L, Semotiuk A, Zafar M, Ahmad M, Sultana S, Liu QR, Zada MP Abidin SZU, Yaseen G. Ethnopharmacological documentation of medicinal plants used for hypertension among the local communities of DIR Lower, Pakistan. J Ethnopharmacol 2015; 175: 138-146

[67] Hussain S, Murtaza G, Mehmood A, Qureshi RA. Conservation of indigenous knowledge of medicinal plants of Western Himalayan region Rawalakot, Azad Kashmir, Pakistan. Pak J Pharm Sci 2017; 30: 773-782

[68] Bibi R, Tariq A, Mussarat S, Khan SN, Rahman H, Allah EFA, Ullah R, Adnan M. Ethnomedicinal, phytochemical and antibacterial activities of medicinal flora of Pakistan used against Pseudomonas aeruginosa - a review. Pak J Pharm Sci 2017; 30: 2285-2300

[69] Habiba U, Ahmad M, Shinwari S, Sultana S, Shinwari ZK, Zafar M. Antibacterial and antifungal potential of Himalayan medicinal plants for treating wound infections. Pak J Bot 2016; 48: 371-375

[70] Savona G, Piozzi F, Hanson JR, Siverns M. Structures of three new diterpenoids from Ballota species. J Chem Soc Perkin I 1977: 322-324

[71] Rustaiyan A, Mosslemin-Kupaii MH, Zdero C. Furolabdanes and related compounds from Ballota aucheri. Phytochemistry 1992; 31: 344-346

[72] Yilmaz BS, Çitoğlu GS. High performance liquid chromatographic analy sis of some diterpenoids of the Ballota species. FABAD J Pharm Sci 2003; 28: $13-17$

[73] Çitoğlu GS, Yilmaz BS, Tarikahya B, Tipirdamaz R. Chemotaxonomy of Ballota species. Chem Nat Compd 2005; 41: 299-302

[74] Rustaiyan A, Mosslemin-Kupaii MH, Papastergiou F, Jakupovic J. Persianone, a dimeric diterpene from Ballota aucheri. Phytochemistry 1995; 40: $875-879$

[75] Yilmaz BS, Çitoğlu GS. Chemical constituents of Ballota L. species. Ankara Universitesi Eczacilik Fakultesi Dergisi 2003; 32: 37-53

[76] Çitoğlu GS, Sever B, Antus S, Baitz-Gács E, Altanlar N. Antifungal diterpenoids and flavonoids from Ballota inaequidens. Pharm Biol 2004; 42: 659-663 
[77] Savona G, Piozzi F, Hanson JR. 13-Hidroxyballonigrinolide, a new diterpenoid from Ballota lanata. Phytochemistry 1978; 17: 2132-2133

[78] Savona G, Piozzi F, Hanson JR, Siverns M. New diterpenoids from species of genus Ballota. Chim Ind 1976; 58: 378

[79] Savona G, Bruno M, Piozzi F, Barbagallo C. Diterpenes from Ballota species. Phytochemistry 1982; 21: 2132-2133

[80] Çitoğlu G, Tanker M, Sever B, Englert J, Anton R, Altanlar N. Antibacterial activities of diterpenoids isolated from Ballota saxatilis subsp. saxatilis. Planta Med 1998; 40: 484-485

[81] Hussein AA, Jimeno ML, Rodríguez B. Structural and spectral assignment of a new diterpenoid isolated from Ballota undulata and complete $\mathrm{H}$ and C NMR data assignment for three other structurally related compounds. Mag Res Chem 2007; 45: 899-901

[82] Al-Musayeib NM, Abbas FA, Ahmad MS, Mossa JS, El-Feraly FS. Labdane diterpenes from Otostegia fruticosa. Phytochemistry 2000; 54: 771-775

[83] Farooq U, Khan A, Khan AF, Khan SS, Sarwar R, Ahmad VU, Waseem A. Two new ballonigrin-type diterpenoids from the roots of Ballota limbata. Nat Prod Commun 2012; 7: 149-150

[84] Savona G, Piozzi F, Hanson JR, Siverns M. The structure of ballotenol, a new diterpenoid from Ballota nigra. J Chem Soc Perkin I 1977: 497-499

[85] Savona G, Piozzi F, Hanson JR, Siverns M. Structure of ballotinone, a diterpenoid from Ballota nigra. J Chem Soc Perkin I 1976: 1607-1609

[86] Prakash O, Bhakuni DS, Kapil RS, Rao GSRS, Ravindranath B. Diterpenoids of Roylea calycina (Roxb.) Briq. J Chem Soc Perkin I 1979: 13051308

[87] Sharma R, Chebolu R, Ravikumar PC. Isolation and structural elucidation of two new labdane diterpenoids from the aerial part of Roylea cinerea. Phytochemistry Lett 2015; 13: 187-193

[88] Sharma R, Mohammadi-Ostad-Kalayeh S, Stahl F, Zeilinger C, Dräger G, Kirschning A, Ravikumar PC. Two new labdane diterpenoids and one new - lactam from the aerial parts of Roylea cinerea. Phytochemistry Lett 2017; 19: 101-107

[89] Zaabat N, Hay AE, Michalet S, Darbour N, Bayet C, Skandrani I, ChekirGhedira L, Akkal S, Dijoux-Franca MG. Antioxidant and antigenotoxic properties of compounds isolated from Marrubium deserti de Noé. Food Chem Toxicol 2011; 49: 3328-3335

[90] Zaabat N, Darbour N, Bayet C, Michalet S, Doléans-Jordheim A, ChekirGhedira L, Akkal S, Dijoux-Franca MG. Preliminary study of Marrubium deserti de Noé, an endemic Algerian Lamiaceae. Phytotherapie 2010; 8: 353-358

[91] Davies-Coleman MT, Rivett DEA. Transformation of hispanolone from Ballota africana into 15, 16-epoxy-9-hydroxylabda-13(16), 14-diene. S Afr J Chem 1990; 43: 117-119

[92] Savona G, Piozzi F, Rodriguez B. Hispanolone, a new furanoditerpene. Heterocycles 1978; 9: 257-261

[93] Seidel V, Bailleul F, Tillequin F. Isolation from Ballota nigra L. of 13-hydroxyballonigrinolide, a diterpene useful for the standardization of the drug. J Pharm Belg 1996; 51: 72-73

[94] Seidel V, Bailleul F, Tillequin F. Diterpene et esters heterosidiques phenylpropanoiques de Ballota nigra. Ann Pharm Fr 1998; 56: 31-35

[95] Savona G, Piozzi F, Hanson JR, Siverns M. 18-Hidroxyballonigrin, a new diterpenoid from Ballota acetabulosa. J Chem Soc Perkin I 1978: 12711272

[96] Bruno M, Bondí ML, Piozzi F, Arnold NA, Simmonds MS]. Occurrence of 18-hydroxyballonigrine in Ballota saxatilis ssp. saxatilis from Lebanon. Biochem System Ecol 2001; 29: 429-431

[97] Rustaiyan A, Mosslemin-Kupaii MH, Habibi Z. Two new diterpenes from Ballota aucheri. Iran J Chem Chem Eng 1991; 10: 72-77

[98] Ali MS, Amina M, Al-Lohedan HA, Al Musayeib NM. Human serum albumin binding to the biologically active labdane diterpene "leoheterin": spectroscopic and in silico analysis. J Photochem Photobiol B 2018; 182: $9-17$
[99] Balansard ]. The lactone of some Labiatae. C R Seances Soc Biol 1934; 117: 1014-1015

[100] Tesso H, König WA. Terpenes from Otostegia integrifolia. Phytochemistry 2004; 65: 2057-2062

[101] Endale A, Bisrat D, Animut A, Bucar F, Asres K. In vivo antimalarial activity of a labdane diterpenoid from the leaves of Otostegia integrifolia Benth. Phytother Res 2013; 27: 1805-1809

[102] Bruno M, Savona G, Pascual C, Rodríguez B. Preleosibirin, a prefuranic labdane diterpene from Ballota nigra subsp. foetida. Phytochemistry 1986; 25: 538-539

[103] Savona G, Piozzi F, Marino M. Rupestralic acid, a new lactone. Heterocycles 1977; 7: 161-164

[104] Rodriguez B, Savona G, Piozzi F. Two new unusual diterpenoids from Ballota hispanica. J Org Chem 1979; 44: 2219-2221

[105] López de Lerma J, García-Blanco S, Rodríguez JG. New compounds from Ballota hispanica. X-ray crystal and molecular structure of hispanonic acid methyl ester. Tetrahedron Lett 1980; 21: 1273-1274

[106] Riaz M, Krohna K, Malik A, Flörke U. Limbetazulone: a new 'decahydro8-oxanaphtho[2, 1-f]-azulen-7-one' diterpenoid from Ballota limbata, and occurence of two conformational isomers in the crystal. Chem Biodivers 2004; 1: 458-462

[107] Ahmad VU, Farooq U, Abbaskhan A, Hussain J, Abbasi MA, Nawaz SA, Choudhary MI. Four new diterpenoids from Ballota limbata. Helv Chim Acta 2004; 87: 682-689

[108] Ayatollahi SA, Kobarfard F, Asgarpanah J, Rahmati Roodsari M, Fana Gh, Iqbal Choudhary M. Diterpenoids of Otostegia persica (Burm.) Boiss. DARU J Pharm Sci 2009; 17: 290-293

[109] Farooq U, Khan A, Ahmad VU, Khan SS, Kousar F, Arshad S. Two new rare-class tetracyclic diterpenoids from Otostegia limbata. Chem Pharm Bull 2007; 55: 471-473

[110] Ahmad VU, Farooq U, Hussain J, Ullah F, Nawaz SA, Choudhary MI. Two new diterpenoids from Ballota limbata. Chem Pharm Bull 2004; 52 : $441-443$

[111] Farooq U, Khan A, Ahmad VU, Azhar-Ul-Haq, Khan SS, Farooq R, Pervez $\mathrm{A}$, Shah MM. Complete ${ }^{1} \mathrm{H}$ and ${ }^{13} \mathrm{C}$ NMR assignments of two new transclerodane diterpenoids from Otostegia limbata. Magn Reson Chem 2007; 45: 766-769

[112] Ahmad VU, Khan A, Farooq U, Kousar F, Khan SS, Nawaz SA, Abbas MA, Choudhary MI. Three new cholinesterase-inhibiting cis-clerodane diterpenoids from Otostegia limbata. Chem Pharm Bull 2005; 53: 378381

[113] Ahmad VU, Khan A, Farooq U, Kousar F, Khan SS, Hussain J. Two new trans-clerodane diterpenoids from Otostegia limbata. J Asian Nat Prod Res 2007; 9: 91-95

[114] Farooq U, Khan A, Ahmad VU, Kousar F, Iqbal S. Limbatolide F and G: two new trans-clerodane diterpenoids from Otostegia limbata. Polish J Chem 2005; 79: 1757-1762

[115] Tóth E, Tóth G, Máthé I, Blunden G. Martynoside, forsythoside B, ladanein and $7 \alpha$-acetoxyroyleanone from Ballota nigra L. Biochem System Ecol 2007; 35: 894-897

[116] Kumar S, Dobhal MP, Joshi BC, Baror FSK. Chemical investigation and pharmacological screening of Roylea elegans Wall. Part Wall. Part II. Herba Polonica 1981; 27: 99-102

[117] Shabnam Habib R, Jamshaid $M$, Tahir MN, Khan T], Khan IU. $(4 R, 5 R, 6 S, 7 R, 8 S, 9 R, 10 S, 13 S)-7,8 \beta$-Epoxy-momilactone-A. Acta Crystallogr E 2008; 64: 0892

[118] Gray CA, Rivett DEA, Davies-Coleman MT. The absolute stereochemis try of a diterpene from Ballota aucheri. Phytochemistry 2003; 63: 409413

[119] Harrison L], Asakawa Y. Methyl verticoate, a novel diterpenoid with an unusual carbon skeleton from the conifer Sciadopitys verticillata. J Chem Res 1988; 10: 340-341 
[120] Wu S], Chan HH, Hwang TL, Qian K, Morris-Natschke S, Lee KH, Wu TS. Salviatalin A and salvitrijudin A, two diterpenes with novel skeletons from roots of Salvia digitaloides and anti-inflammatory evaluation. Tetrahedron Lett 2010; 51: 4287-4290

[121] Wu SJ, Huang CH, Chan YY, Liao YR, Hwang TL, Wu TS. Two diterpenoids and a cyclopenta[c]pyridine derivative from roots of Salvia digitaloids. Int J Mol Sci 2014; 15: 11566-11577

[122] Wang LX, Fang YD, Zhang RH, Ren FC, Zhang XJ, Wang F, Xiao WL. Hispanane-type diterpenoid and secoiridoid glucosides from Viburnum cylindricum. Chem Biodivers 2018; 15: e1700418

[123] Herz W, Prasad JS, Mohanraj S. Cationic cyclizations and rearrangements as models for strobane and hispanane biogenesis. J Org Chem 1983; 48: 81-90

[124] Karioti A, Heilmann J, Skaltsa H. Labdane diterpenes from Marrubium velutinum and Marrubium cylleneum. Phytochemistry 2005; 66: 10601066

[125] Hersel U, Steck M, Seifert K. A new route to 2,7- and 7-functionalized labdanes. Eur J Org Chem 2000: 1609-1615

[126] Tasdemir D, Wright AD, Sticher O. New furanoid and seco-labdanoid diterpenes from Leonurus persicus. J Nat Prod 1996; 59: 131-134

[127] Hussein AA, Meyer MJ], Rodriguez B. Complete ${ }^{1} \mathrm{H}$ and ${ }^{13} \mathrm{C}$ NMR assignments of three labdane diterpenoids isolated from Leonotis ocymifolia and six other related compounds. Magn Reson Chem 2003; 41: 147151

[128] El Bardai S, Morel N, Wibo M, Fabre N, Llabras G, Lyoussi B, QuetinLeclerrca J. The vasorelaxxant activity of marrubenol and marrubin from Marrubium vulgare. Planta Med 2003; 69: 75-77

[129] Rigano D, Aviello G, Bruno M, Formisano C, Rosselli S, Capasso R, Senatore F, Izzo AA, Borrelli F. Antispasmodic effects and structureactivity relationship of labdane diterpenoids from Marrubium globosum ssp. libanoticum. J Nat Prod 2009; 72: 1477-1481

[130] Tesso $\mathrm{H}$. Isolation and Structure Elucidation of natural Products from Plants [Dissertation]. Hamburg: University of Hamburg, Institute of Organic Chemistry; 2005

[131] Rodriguez B, Savona G. Complete assignments of ${ }^{1} \mathrm{H}$ and ${ }^{13} \mathrm{C}$ NMR spectra of four hispanane diterpenoids. Magn Reson Chem 2000; 38 : 802-804

[132] Saeidnia S, Ghamarinia M, Gohari AR, Shaker A. Terpenes from the root of Salvia hypoleuca Benth. DARU J Pharm Sci 2012; 20: 66

[133] Wellsow J, Grayer JR, Veitch NC, Kokubun T, Lelli R, Kite GC, Simmonds MSJ. Insect-antifeedant and antibacterial activity of diterpenoids from species of Plectranthus. Phytochemistry 2006; 67: 1818-1825

[134] Meriçli AH, Meriçli F, Tuzlacı E. Flavonoids of Ballota acetabulosa. Acta Pharmaceutica Turcica 1988; 30: 143-144

[135] Askun T, Tekwu EM, Satil F, Modanlioglu S, Aydeniz H. Preliminary antimycobacterial study on selected Turkish plants (Lamiaceae) against Mycobacterium tuberculosis and search for some phenolic constituents. BMC Complement Altern Med 2014; 13: 365

[136] Ferreres F, Tomas-Barberan FA, Tomas-Lorente F. Flavonoid compounds from Ballota hirsuta. J Nat Prod 1986; 49: 554-555

[137] Tomás-Barberan FA, Gil MI, Ferreres F, Tomás-Lorente F. Flavonoid pcoumaroylglucosides and 8-hydroxyflavone allosylglucosides in some Labiatae. Phytochemistry 1992; 31: 3097-3102

[138] Jiang S, Bai XR, Zou DZ, Wang P, Zhang L, Long P, Zhang CH, He YL, Li $\mathrm{ZH}$, Li MH. Chemical constituents from Panzeria alaschanica Kupr. (Labiatae). Biochem System Ecol 2015; 61: 268-270

[139] Zhukov IM, Belikov VV. Chromatospectrophotometric determination of flavonoids and dioxycinnamic acids in the above-ground part of Ballota nigra L. Farmatsevtichnii Zhurnal (Kiev) 1989; 5: 55-58

[140] Ku CT, Chen SC, Wang JP, Wu JB, Kuo SC. Studies on anti-inflammatory constituents of Leucas mollissima Wall. var. chinensis Benth. Chin Pharm J 2000; 52: 261-273
[141] Tofighi Z, Alipour F, Hadavinia H, Abdollahi M, Hadjiakhoondi A, Yassa N. Effective antidiabetic and antioxidant fractions of Otostegia persica extract and their constituents. Pharm Biol 2014; 52: 961-966

[142] Tofighi Z, Alipour F, Goodarzy S, Yassa N, Hadjiakhoondi A, Hadavinia $\mathrm{H}$. Phytochemical and antidiabetic investigations of Otostegia persica essential oil from Iran. Planta Med 2009; 75: 1013-1014

[143] Hou FF, Zheng YF, Zhang HM, Shen SM, Du SS. Chemical constituents in Panzerina alaschanica Kupr. Zhongguo Shiyan Fangjixue Zazhi 2009; 15: $18-20$

[144] Wang QH, Hu YR, Shao CY, Wu JS, Han NRCKT, Dai NYT, Wu RJ. Antiinflammatory effect and high performance liquid chromatography (HPLC) analysis of the EtOAc extract from Panzeria alaschanica. Afr J Pharm Pharmacol 2015; 9: 974-981

[145] Shao C, Wang Q, Wu R, Wu J, Han N, Dai N. Study on chemical constituents of Panzeria alaschanica. J Chin Med Mat 2015; 38: 1668-1670

[146] Çitoglu G, Tanker M, Sever B. Flavonoid aglycones from Ballota saxatilis subsp saxatilis. Pharm Biol 1999; 37: 158-160

[147] Çitoğlu GS, Sever B, Antus S, Baitz-Gács E, Altanlar N. Antifungal flavonoids from Ballota glandulosissima. Pharm Biol 2003; 41: 483-486

[148] Abdelshafeek KA, Daboob AA. Investigation of flavonoids and antimicrobial activity of Ballota andreuzziana. Pharmacogn Mag 2009; 5: 203-208

[149] Abdelshafeek KA, Elgendy HA, El Missiry MM, Seif El Nasr MM. Struc ture elucidation of phenolic acids, flavonoids and hypocholesterolemic activity of Nepeta septemcrenata and Otostegia fruticosa. Der Pharma Chem 2016; 8: 357-362

[150] Siciliano T, Bader A, Vassallo A, Braca A, Morelli I, Pizza C, De Tommasi N. Secondary metabolites from Ballota undulata. Biochem System Ecol 2005; 33: 341-351

[151] Ikram M, Jehangir S, Razaq S, Kawano M. Chemical investigation of Otostegia limbata. Planta Med 1978; 37: 189-190

[152] Parvez M, Riaz M, Malik A. Eupatorin. Acta Crystallogr E 2001; E57: o289-0291

[153] Ayatollahi SAM, Kobarfard F, Asgarpanah J, Choudhary MI. Antiglycation activity of Otostegia persica (Burm.) Boiss. Afr J Biotechnol 2010; 9: 3645-3648

[154] Ansari S, Joshi YC, Dobhal MP, Joshi BC. Chemical constituents of the stem of Roylea elegans Wall. Pharmazie 1982; 37: 70-71

[155] Kisiel W, Piozzi F. Tangeretin from Ballota nigra. Polish J Chem 1995; 69: 476-477

[156] Aboutabl EA, Sokkar NM, Awaad AS. Phytochemical study of Otostegia fruticosa (Forssk.) and biological activity. Bull Fac Pharm Cairo Univ 2000; 38: 115-118

[157] Ghedadba N, Hambaba L, Ayachi A, Aberkane MC, Bousselsela H, Oueld-Mokhtar SM. Polyphenols content, antioxidant and antimicrobial activities of leaf extracts of Marrubium deserti de Noé ex Coss. Phytotherapie 2015; 13: 118-129

[158] Yassa N, Sharififar F, Shafiee A. Otostegia persica as a source of natural antioxidants. Pharm Biol 2005; 43: 33-38

[159] Uysal S, Aumeeruddy-Elalfi Z, Zengin G, Aktumsek A, Mocan A, Custodio L, Neng NR, Nogueira JMF, Ćirić A, Glamočlija J, Soković M, Mahomoodally MF. Insight into the biological properties and phytochemical composition of Ballota macrodonta Boiss. et Balansa, - an endemic medicinal plant from Turkey. Ind Crops Prod 2018; 113: 422 428

[160] Shrififar F, Yassa N, Shafiee A. Antioxidant activity of Otostegia persica (Labiatae) and its constituents. Iran J Pharm Res 2003; 2: 235-239

[161] Sahpaz S, Skaltsounis AL, Bailleul F. Polyphenols from Ballota acetabulosa. Biochem System Ecol 2002; 30: 601-604

[162] Hennebelle T, Sahpaz S, Ezer N, Bailleul F. Polyphenols from Ballota larendana and Ballota pseudodictamnus. Biochem System Ecol 2008; 36: $441-443$ 
[163] Darbour N, Baltassat F, Raynaud J. The presence of an O-heteroside and a C-heteroside of apigenine in leaves of Ballota-foetida Lamk (Labiatae). Pharmazie 1986; 41: 605-606

[164] Radwan HM, El-Missiry MM, El-Nasr MM. Phytochemical investigation of Ballota undulata Benth. Bull Fac Pharm Cairo Univ 1997; 35: 83-86

[165] Ranganayaki S, Gusain PS, Sigh AK. The chemical constituents of leaves of Roylea elegans. J Sci Res Plants Med 1985; 6: 5-9

[166] Tofighi Z, Ostad SN, Khezrrahdoost S, Salehizadeh H, Yassa N. Potent anti-nociceptive and anti-inflammatory effects of methanol fraction of Otostegia persica extract and its components. Res J Pharmacogn 2017; 4: 23-29

[167] Khan A, Ahmad VU, Farooq U, Bader S, Arshad S. Two new flavonol glycosides from Otostegia limbata Benth. Chem Pharm Bull 2009; 57 : 276-279

[168] Wang QH, Wu JS, Wu RJ, Han NRCKT, Dai NYT. Anti-inflammatory effect and isolation of phenylethanoid and acylated flavone glycosides from Panzeria alaschanica. Z Naturforsch B Chem Sci 2015; 70: 379384

[169] Bao ML, Wang QH, Hao JS. In vivo antioxidative activity of 5,7,4'-trihydroxyflavone-7-O-(6"'-O-[E]-coumaroyl)- $\beta$-glucopyranoside isolated from Panzeria alaschanica. Afr J Pharm Pharmacol 2017; 11: 567-572

[170] Bertrand MC, Tillequin F, Bailleul F. Two major flavonoids from Ballota nigra. Biochem System Ecol 2000; 28: 1031-1033

[171] Noor AT, Fatima I, Ahmad I, Malik A, Afza N, Iqbal L, Latif M, Khan SB. Leufolins $A$ and $B$, potent butyrylcholinesterase-inhibiting flavonoid glucosides from Leucas urticifolia. Molecules 2007; 12: 1447-1454

[172] Khan A, Ahmad VU, Farooq U. Two new acylated flavonol glycosides from the roots of Otostegia limbata. Helv Chim Acta 2009; 92: 731-739

[173] Wang Q, Wu J, Wu X, Tai W, Dai N, Wu R, Han N. Anti-inflammatory and analgesic effects of two new flavone C-glycosides from Panzeria alaschanica. Monatsh Chem 2015; 146: 1025-1030

[174] Anwar KJ, Mosihuzaman M, Nahar N, Khan SH, Rashid R, Sultana N. Chemical studies of anti-hyperglyceamic active extract of Otostegia aucheri Bioss. J Bangladesh Chem Soc 2004; 17: 18-22

[175] Abdelshafeek K, Elgendy HA, ElMissiry MM, Seif ELNasr MM. Isolation and identification of some chemical constituents and antimicrobial activity of two Lamiaceae plants growing in Saini. Egypt J Chem 2016; 59: 21-31

[176] Dobhal MP, Joshi BC. Chemical investigations of Roylea elegans Wall. Part I. Herba Polonica 1979; 25: 95-97

[177] Ayatollahi SAM, Kobarfard F, Asgarpanah J, Ahmed Z. Chemical constituents from Otostegia persica. J Chem Soc Pak 2007; 29: 61-63

[178] Majumder PL, Maiti RN, Panda SK, Mal D, Raju MS, Wenkert E. Structure of moronic acid. J Org Chem 1979; 44: 2811-2812

[179] Janicsak G, Veres K, Zoltan Kakasy A, Mathe I. Study of the oleanolic and ursolic acid contents of some species of the Lamiaceae. Biochem System Ecol 2006; 34: 392-396

[180] Ranganayaki S, Gusain PS, Singh AK. Isolation and study of a pentacyclic triterpene from the roots of Roylea elegans Wall. J Sci Res Plants Med 1979; 1: 44-47

[181] Fatima I, Ahmad I, Anis I, Malik A, Afza N, Iqbal L, Latif M. New butyrylcholinesterase inhibitory steroid and peroxy acid from Leucas urticifolia. Arch Pharm Res 2008; 31: 999-1003

[182] Bhatt UP, Sati SC, Bahuguna RP, Semwal RB, Semwal DK. Two antidiabetic constituents from Roylea cinerea (D. Don) Baill. Nat Prod Res 2018; 32: 1281-1286

[183] Popa DP, Pasechnik GS. Higher terpenoids of some species of Labiatae. Chem Nat Compd 1974; 10: 529-530

[184] Dixit V, Irshad S, Singh H, Agnihotri P, Husain T, Khatoon S. High-performance thin-layer chromatographic determination of three therapeutic phenolic components in Leucas species. J Planar Chromatogr Modern TLC 2017; 30: 25-31
[185] Vrchovská V, Spilková ], Valentão P, Sousa C, Andrade PB, Seabra RM. Antioxidative properties and phytochemical composition of Ballota nigra infusion. Food Chem 2007; 105: 1396-1403

[186] Tóth E, Janicsák G, Máthé I, Blunden G. Determination of phenylpropanoids in three Ballota species. J Planar Chromatogr - Modern TLC 2009; 22: 293-296

[187] Didry N, Seidel V, Dubreuil L, Tillequin F, Bailleul F. Isolation and antibacterial activity of phenylpropanoid derivatives from Ballota nigra. J Ethnopharmacol 1999; 67: 197-202

[188] Blunden G, Yang MHE, Yuan ZX, Smith BE, Patel A, Cegarra JA, Mathé I, Janicsàk G. Betaine distribution in the Labiatae. Biochem System Ecol 1996; 24: 71-81

[189] Janicsák G, Tóth E, Máthé I. TLC-densitometric investigations of phenylpropanoid glycosides in black horehound (Ballota nigra L.). J Planar Chromatogr - Modern TLC 2007; 20: 443-446

[190] Tipirdamaz R, Guvenc A. Seed fatty acids composition of Ballota cristata. Chem Nat Compd 2004; 40: 291-292

[191] Balansard J. Pharmacological study of Ballota foetida. C R Seances Soc Biol 1934; 115: 1295-1297

[192] Khokhrina TA, Peshkova VA. Stachydrine from Phlomis tuberosa and Panzeria lanata. Chem Nat Compd 1975; 10: 284

[193] Seidel V, Bailleul F, Tillequin F. Phenylpropanoid glycosides from Balloto nigra. Planta Med 1996; 62: 186-187

[194] Seidel V, Bailleul F, Libot F, Tillequin F. A phenylpropanoid from Ballota nigra. Phytochemistry 1997; 44: 691-693

[195] Pedersen JA. Distribution and taxonomic implications of some phenolics in the family Lamiaceae determined by ESR spectroscopy. Biochem System Ecol 2000; 28: 229-253

[196] Ertaş A, Boğa M, Yeşil Y. Phytochemical profile and ABTS cation radical scavenging, cupric reducing antioxidant capacity and anticholinesterase activities of endemic Ballota nigra L. subsp. anatolica P.H. Davis from Turkey. J Coast Life Med 2014; 2: 555-559

[197] Piault L. The lactone of some Labiatae. J Pharm Chim 1911; 1: 248-255

[198] Kolisnyk Y, Ochkur O, Kovalyova A, Koshoviy O. Organic acids of vegetative and generative organs of black horehound. Pharma Innovation 2014; 3: 33-37

[199] Marin PD, SajdI V, Kapor S, Tatic B, Petkovic B, Duletic S. Fatty acids of the Stachyoideae. Biochem System Ecol 1992; 20: 389-392

[200] Abdelshafeek KA, Elgattar AA, Zarkoon AH, Alwahash MA, Shahat AA. Investigation of the volatile oils, lipid constituents and biological activity of Ballota andreuzziana, Teucrium zanonii and Verbena tenuisecta in Libya. Asian Pac J Tropical Med 2010; 3: 594-601

[201] Rustaiyan A, Masoudi S, Ameri N, Samiee K, Monfared A. Volatile constituents of Ballota aucheri Boiss., Stachys benthamiana Boiss. and Perovskia abrotanoides Karel. growing wild in Iran. J Ess Oil Res 2006 . 18: 218-221

[202] Laouer H, Yabrir B, Djeridane A, Yousfi M, Beldovini N, Lamamra M. Composition, antioxidant and antimicrobial activities of the essential oil of Marrubium deserti. Nat Prod Commun 2009; 4: 1133-1138

[203] Chebrouk F, Hadj Mahammed M. Composition des huiles essentielles de Marrubium deserti de Noe de la region de Ghardaïa. Annales de la Faculté des Sciences et Sciences de I'Ingénieur 2009; 1: 75-81

[204] Chemsa AE, Zellagui A, Öztürk M, Erol E, Ceylan O, Duru ME, Gherraf N Antibiofilm formation, antioxidant and anticholinesterase activities of essential oil and methanol extract of Marrubium deserti de Noé. J Mater Environ Sci 2018; 7: 993-1000

[205] Riccobono L, Ben Jemia M, Senatore F, Bruno M. Chemical composition and biological activity of the essential oil of Ballota hispanica (L.) Benth. growing wild in Sicily. Plant Biosyst 2016; 150: 1065-1071

[206] Todorova M, Altantsetseg S, Trendafilova A, Shatar S. Chemical composition of Panzeria lanata (L.) Bunge essential oil from Mongolian Gobi. J Ess Oil-Bearing Plants 2013; 16: 257-260 
[207] Đorđević AS, Jovanović OP, Zlatković BK, Stojanović GS. Chemical composition of Ballota macedonica Vandas and Ballota nigra L. ssp. foetida (Vis.) Hayek essential oils - the chemotaxonomic approach. Chem Biodivers 2016; 13: 782-788

[208] Morteza-Semnani K, Saeedi M, Akbarzadeh M. The essential oil composition of Ballota nigra. Chem Nat Compd 2007; 43: 722-723

[209] Vukovic N, Sukdolak S, Solujic S, Niciforovic N. Antimicrobial activity of the essential oil obtained from roots and chemical composition of the volatile constituents from the roots, stems, and leaves of Ballota nigra from Serbia. J Med Food 2009; 12: 435-441

[210] Jamzad M, Rustaiyan A, Jamzad Z, Masoudi S. Essential oil composition of Salvia indica L., Thymus caucasicus Wind. Ex Ronniger subsp. grossheimii (Ronniger) Jalas. and Ballota nigra L. three Labiatae species from Iran. J Ess Oil Bearing Plants 2011; 14: 76-83

[211] Kolisnyk YS, Kovaleva AM, Goryacha OV. The study of the volatile oils composition obtained from vegetative and generative organs of Ballota nigra L. Visnik Farmatsii 2014; 78: 59-62

[212] Karemizadeh Z, Amini T, Nazari F, Habibi Z. Volatile constituents of Ballota nigra subsp. anatolica from Iran. Chem Nat Compd 2009; 45: 737738

[213] Sarikaya AG. Volatile component composition of Ballota nigra subsp. anatolica at different vegetation periods in Çamlica province of Kütahya city, Turkey. Appl Ecol Env Res 2017; 15: 1929-1933

[214] Kaya A, Kürkçüoğlu M, Dinç M, Doğu S. Compositions of the essential oils of Ballota nigra subsp. uncinata and subsp. anatolica from Turkey. Indian J Pharm Educ Res 2017; 51: S185-S189

[215] Bader A, Caponi C, Cioni PL, Flamini G, Morelli I. Composition of the essential oil of Ballota undulata, B. nigra ssp. foetida and B. saxatilis. Flavour Fragr J 2003; 18: 502-504

[216] Fraternale D, Bucchini A, Giampieri L, Ricci D. Essential oil composition and antimicrobial activity of Ballota nigra L. ssp foetida. Nat Prod Commun 2009; 4: 585-588

[217] Fraternale D, Ricci D. Essential oil composition and antifungal activity of aerial parts of Ballota nigra ssp foetida collected at flowering and fruiting times. Nat Prod Commun 2014; 9: 1015-1018

[218] Rigano D, Marrelli M, Formisano C, Menichini F, Senatore F, Bruno M, Conforti F. Phytochemical profile of three Ballota species essential oils and evaluation of the effects on human cancer cells. Nat Prod Res 2017; 31: 436-444

[219] Majdi M, Dastan D, Maroofi H. Chemical composition and antimicrobial activity of essential oils of Ballota nigra subsp. kurdica from Iran. Jundishapur J Nat Pharm Prod 2017; 12: e36314

[220] Couladis M, Chinou IB, Tzakou O, Loukis A. Composition and antimicrobial activity of the essential oil of Ballota pseudodictamnus L. Bentham. Phytother Res 2002; 16: 723-726

[221] Erdogan EA, Everest A, Sonmez Ö. Antimicrobial activity of the essential oil of Ballota saxatilis subsp. brachyodonta (Boiss) P. H. Davis \& Doroszenko from Turkey. J Ess Oil Bearing Plants 2014; 17: 1100-1104

[222] Awadh Ali NA, Alhamzy EH, Chhetri BK, Dosoky NS, Setzer WN. Chemical composition, antimicrobial, and cytotoxic activities of the essential oil of Otostegia fruticosa subsp. schimperi from Yemen. Nat Prod Commun 2017; 12: 969-972

[223] Özek T, Tabanca N, Demirci F, Wedge DE, Başer KHC. Enantiomeric distribution of some linalool containing essential oils and their biological activities. Rec Nat Prod 2010; 4: 180-192

[224] Aboutabl EA, Sokkar NM, Megid RMA, De Pooter HL, Masoud H. Composition and antimicrobial activity of Otostegia fruticosa Forssk. oil. J Ess Oil Res 1995; 7: 299-303

[225] Karami A, Esmaeili H, Shahbazian D. Essential oil composition of Rydingia michauxii (Briq.) Scheen \& V.A. Albert endemic of Iran. Nat Prod Res 2018; 32: 229-233
[226] Javidnia K, Miri R, Soltani M, Khosravi AR. Chemical constituents of the essential oil of Otostegia michauxii Briq. from Iran. J Ess Oil Res 2010; 22: $1-2$

[227] Javidnia K, Miri R, Soltani M. Essential oil composition of Otostegia persica Boiss. from Iran. J Ess Oil Res 2010; 22: 609-610

[228] Tofighi Z, Alipour F, Yassa N, Hadjiakhoondi A, Hadavinia H, Goodarzy S, Golestani R. Chemical composition and antioxidant activity of Otostegia persica essential oil from Iran. Int J Essen Oil Ther 2009; 3: 45-48

[229] Sharififar F, Mozaffarian V, Moradkhani S. Comparison of antioxidant and free radical scavenging activities of the essential oils from flowers and fruits of Otostegia persica Boiss. Pak J Biol Sci 2007; 10: 3895-3899

[230] Erdogan-Orhan I, Sever-Yılmaz B, Altun ML, Saltan G. Radical quenching activity, ferric-reducing antioxidant power, and ferrous ion-chelating capacity of 16 Ballota species and their total phenol and flavonoid contents. J Med Food 2010; 13: 1537-1543

[231] Citoglu GS, Çoban T, Sever B, Işcan M. Antioxidant properties of Ballota species growing in Turkey. J Ethnopharmacol 2004; 92: 275-280

[232] Rashid R, Murtaza G, Khan AK, Mir S. Antioxidant and hypoglycemic effect of Otostegia aucheri methanolic extract in streptozotocininduced diabetic male Long-Evans rats. Acta Pol Pharm 2014; 71: 631-635

[233] Sharma R, Yadav D, Asif M, Jayasri MA, Agnihotri VK, Ravikumar PC. Antidiabetic and antioxidant activities of Roylea cinerea extracts: a comparative study. Indian J Exp Biol 2017; 55: 611-621

[234] Kechar K, Hellal B. Evaluation of the antioxidant activity of extracts of Ballota hirsuta Benth. from Tessala (Western Algeria). Phytotherapie 2017; 15: 217-221

[235] Makowczynska J, Grzegorczyk-Karolak I, Wysokinska H. Antioxidant activity of tissue culture-raised Ballota nigra L. plants grown ex vitro. Acta Pol Pharm 2015; 72: 769-775

[236] Daels-Rakotoarison DA, Seidel V, Gressier B, Brunet C, Tillequin F, Bailleul F, Luyckx M, Dine T, Cazin M, Cazin JC. Neurosedative and antioxidant activities of phenylpropanoids from Ballota nigra. Arzneimittelforschung 2000; 50: 16-23

[237] Seidel V, Verholle M, Malard Y, Tillequin F, Fruchart JC, Duriez P, Bailleu F, Teissier E. Phenylpropanoids from Ballota nigra L. Inhibit in vitro LDL Peroxidation. Phytother Res 2000; 14: 93-98

[238] Gursoy N, Tepe B. Determination of the antimicrobial and antioxidative properties and total phenolics of two "endemic" Lamiaceae species from Turkey: Ballota rotundifolia L. and Teucrium chamaedrys C. Koch. Plant Foods Hum Nutr 2009; 64: 135-140

[239] Couladis M, Tzakou O, Verykokidou E, Harvala C. Screening of some Greek aromatic plants for antioxidant activity. Phytother Res 2003; 17: 194-195

[240] Chekol YA, Desta ZY. Determination of antioxidant and antimicrobia activities of leaf extracts of Otostegia integrifolia. Chem Cent J 2018; 12: 63

[241] Waheed I, Ahmad M, Syed NH, Ashraf R. Investigation of phytochemical and antioxidant properties of methanol extract and fractions of Ballota limbata (Lamiaceae). Indian J Pharm Sci 2014; 76: 251-256

[242] Ahmed D, Arshad MA, Asghar MN, Aujla MI. Antioxidant and free radical scavenging potential of Otostegia limbata. Asian J Chem 2010; 22: 4524-4532

[243] Moein S, Pimoradloo E, Moein M, Vessal M. Evaluation of antioxidant potentials and $\alpha$-amylase inhibition of different fractions of Labiatae plants extracts: as a model of antidiabetic compounds properties. Biomed Res Int 2017; 7319504: 1-8

[244] Safaeian L, Yaghoobi S, Javanmard SH, Ghasemi-Dehkordi N. The effect of hydroalcoholic extract of Otostegia persica (Burm.) Boiss. against $\mathrm{H}_{2} \mathrm{O}_{2}$-induced oxidative stress in human endothelial cells. Res J Pharmac 2017; 4: 51-58 
[245] Özbek H, Çitoğlu GS, Dülger H, Uğraş S, Sever B. Hepatoprotective and anti-inflammatory activities of Ballota glandulosissima. J Ethnopharmacol 2004; 95: 143-149

[246] Yilmaz BS, Özbek H, Çitoğlu GS. Antinociceptive and anti-inflammatory activities of Ballota inaequidens. Pharm Biol 2006; 44: 636-641

[247] Elmezogi ], Zetrini A, Ben-Hussein G, Anwair M, Gbaj A, El-Ashheb M, Nahar L, Sarker SD. Evaluation of anti-inflammatory activity of some Libyan medicinal plants in experimental animals. Arch Biol Sci 2012; 64: 1058-1064

[248] Nabil G, Leila H. Anti-inflammatory and analgesic activities of methanolic extract from Marrubium deserti leaves and evaluation of their acute toxicity. Pharm Lett 2016; 8: 33-40

[249] Dulger B, Kilcik MA. Antibacterial activity of Ballota acetabulosa against methicillin-resistant Stapylococcus aureus. Asian J Chem 2011; 23: 416418

[250] Dulger B, Dulger G. Antimicrobial activity of the leaves of Ballota acetabulosa on microorganisms isolated from urinary tract infection. Turk J Pharm Sci 2012; 9: 257-262

[251] Cock IE, van Vuuren SF. The potential of selected South African plants with anti-Klebsiella activity for the treatment and prevention of Ankylosing spondylitis. Inflammopharmacology 2014; 23: 21-35

[252] Quave CL, Plano LRW, Bennett BC. Quorum sensing inhibitors of Staphylococcus aureus from Italian medicinal plants. Planta Med 2011; 77: 188-195

[253] Ullah N, Ahmad I, Ayaz S. In vitro antimicrobial and antiprotozoal activities, phytochemical screening and heavy metals toxicity of different parts of Ballota nigra. BioMed Res Int 2014: 321803

[254] Quave CL, Plano LRW, Pantuso T, Bennett BC. Effects of extracts from Italian medicinal plants on planktonic growth, biofilm formation and adherence of methicillin-resistant Staphylococcus aureus. J Ethnopharmacol 2008; 118: 418-428

[255] Dulger G, Aki C, Dulger B. Antimicrobial activity of endemic Ballota nigra subsp anatolica in Turkey. Asian J Chem 2010; 22: 6497-6502

[256] Dulger G, Dulger B. Antibacterial activity of endemic Ballota nigra subsp. anatolica against some human eye pathogens from Turkey. Int J Life Sci 2017; 5: 1-3

[257] Ullah N, Ahmad I, Ahmad NF. In vitro antimicrobial, antiprotozoal activities and heavy metals toxicity of different parts of Ballota pseudodictamnus (L.) Benth. Pak J Pharm Sci 2017; 30: 2203-2209

[258] Kahaliw W, Aseffa A, Abebe M, Teferi M, Engidawork E. Evaluation of the antimycobacterial activity of crude extracts and solvent fractions of selected Ethiopian medicinal plants. BMC Complement Altern Med 2017; 17: 143

[259] Anwar N, Salik S, Ahmad D. Antibacterial activity of Otostegia limbata. Int J Agric Biol 2009; 11: 647-650

[260] Javidnia K, Miri R, Assadollahi M, Gholami M, Ghaderi M. Screening of selected plants growing in Iran for antimicrobial activity. Iran J Sci Technol A 2009; 33: 329-333

[261] Asghari G, Nourallahi H, Havaie SA, Issa L. Antimicrobial activity of Otostegia persica Boiss. extracts. Res Pharm Sci 2006; 1: 53-58

[262] Nelson K, Lyles JT, Li T, Saitta A, Addie-Noye E, Tyler P, Quave CL. Antiacne activity of Italian medicinal plants used for skin infection. Front Pharmacol 2016; 7: 425-438

[263] Dulger B, Kilcik MA. Antifungal activity of Ballota acetabulosa against yeast Candida and Cryptococcus species. Asian J Chem 2011; 23: 413415

[264] Hashem M. Antifungal properties of crude extracts of five Egyptian medicinal plants against dermatophytes and emerging fungi. Mycopathologia $2011 ; 172: 37-46$

[265] Naz S, Farooq U, Khan A, Khan S, Sarwar R, Mirza N. Physiochemical screening and antimicrobial potential of Otostegia limbata Benth. J Chem Soc Pak 2014; 36: 683-686
[266] Maqsood M, Qureshi R, Ikram M, Ali S, Rafi M, Khan JA, Ahmed MS. Preliminary screening of methanolic plant extracts against human rhabdomyosarcoma cell line from salt range, Pakistan. Pak J Bot 2015; 47: 353-357

[267] Bahuguna RP, Jangwan JS, Singh R, Bhatt UP. In vitro cytotoxic, in vitro and in vivo antidiabetic activity of Roylea cinerea. Sci Revs Chem Commun 2015; 5: 69-76

[268] Marimuthu N, Viswanathan T, Radha M, Suganya J. Computational screening of the phytocompounds from the plant Ballota nigra Linn against the human papillomavirus (HPV) E6. Res J Pharm Technol 2017; 10: 3095-3097

[269] Fronza M, Murillo R, Ślusarczyk S, Adams M, Hamburger M, Heinzmann B, Laufer S, Merfort I. In vitro cytotoxic activity of abietane diterpenes from Peltodon longipes as well as Salvia miltiorrhiza and Salvia sahendica. Bioorg Med Chem 2011; 19: 4876-4881

[270] da Cruz Araujo EC, Lima MAS, Montenegro RC, Nogueira M, CostaLotufo LV, Pessoa C, de Moraes MO, Silveira ER. Cytotoxic abietane diterpenes from Hyptis martiusii Benth. Z Naturforsch C Biosci 2006; 61: 177-183

[271] Zhang Y, Ma R, Cheng S, Gu G. Marrubenol inhibits osteosarcoma cancer cell growth by inducing autophagic cell death and inhibiting cancer cell migration and invasion. J Balkan Union of Oncology 2018; 23: 729734

[272] Xiao C], Liu YC, Luo SH, Hua J, Liu Y, Li SH. Localisation of two bioactive labdane diterpenoids in the peltate glandular trichomes of Leonurus japonicus by laser microdissection coupled with UPLC-MS/MS. Phytochem Anal 2017; 28: 404-409

[273] Ateyyat MA, Al-Mazra'awi M, Abu-Rjai T, Shatnawi MA. Aqueous extracts of some medicinal plants are as toxic as Imidacloprid to the sweet potato whitefly, Bemisia tabaci. J Insect Sci 2009; 9: 15

[274] Al-Alawi MS. Acaricidal activity of medicinal plants against the developmental stage of the two spotted spider mite, Tetranychus urticae (Acari: Tetranychidae). Int J Agr Res 2014; 9: 38-46

[275] Dube FF, Tadesse K, Birgersson G, Seyoum E, Tekie H, Ignell R, Hill SR. Fresh, dried or smoked? Repellent properties of volatiles emitted from ethnomedicinal plant leaves against malaria and yellow fever vectors in Ethiopia. Malar J 2011; 10: 375

[276] Khan J, Anjum SI, Khan I, Rehman FU, Khan A. Larvicidal and development retarding effects of hexane crude extract of Otostegia limbata on 3rd instar larvae of Drosophila melanogaster Meign (Diptera: Drosophilidae). J Entomol Zool Stud 2015; 3: 6-9

[277] Salari E, Ahmadi K, Zamani R. Study on the effects of acetonic extract of Otostegia persica (Labiatae) on three aphid species and one stored product pest. Adv Environ Biol 2010; 4: 346-349

[278] Pascual-Villalobos M], Robledo A. Anti-insect activity of plant extracts from the wild flora in southeastern Spain. Biochem System Ecol 1999; 27: $1-10$

[279] Haq RU, Farooq U, Wahab A, Raza M, Ahmad VU, Khan RA. Investigation of antitussive and toxicological activity of Ballota limbata in mice. Pharm Biol 2011; 49: 627-632

[280] Vural K, Ezer N, Erol K, Sahin FP. Anxioloytic and antidepressant activities of some Ballota species. J Fac Pharm Gazı 1996; 13: 29-32

[281] Hajhashemi V, Rabbani M, Asghari GR, Karami-Saravi Z. Effects of Otostegia persica (Burm.f.) Boiss on morphine withdrawal syndrome in mice. Iranian J Pharm Res 2004; 3: 171-175

[282] Ansari F, Shafaroodi H, Asgarpanah J. Anticonvulsant activity of Otostegia persica (Burm.) Boiss. Biosci Biotechnol Res Asia 2014; 11: 733-737

[283] Çitoğlu GS, Özbek H, Severa B. Antinociceptive activity of Ballota glandulosissima Hub.-Mor \& Patzak. Eastern J Med 2005; 10: 24-28

[284] Nusier MK, Bataineh HN, Bataineh ZM, Daradka HM. Effects of Ballota nigra on glucose and insulin in alloxan-diabetic albino rats. Neuro Endocrinol Lett 2007; 28: 470-472 
[285] Nusier MK, Bataineh HN, Bataineh ZM, Daradka HM. Effects of Ballota nigra on blood biochemical parameters and insulin in albino rats. Neuro Endocrinol Lett 2007; 28: 473-476

[286] Qazan WSh. Hypolipidaemic effects of Ballota undulata in rabbits. Pak J Biol Sci 2008; 11: 1169-1172

[287] Ebrahimpoor MR, Khaksar Z, Noorafshan A. Anti-diabetic effect of orally administered Otostegia persica extract on streptozotocin diabetic rats. Comp Clin Pathol 2011; 20: 523-525

[288] Manzari-Tavakoli A, Pouraboli I, Yaghoobi MM, Mehrabani M, Mirtadzadini SM. Antihyperglycemic, antilipid peroxidation, and insulin secretory activities of Otostegia persica shoot extract in streptozotocin-induced diabetic rats and in vitro C187 pancreatic $\beta$-cells. Pharm Biol 2013; 51: 253-259

[289] Takhtfooladi MA, Asghari A, Hoseinzadeh HA, Mokhtari F. Effect of Otostegia persica extract on ischemia/reperfusion induced renal damage in diabetic rats. A biochemical study. Acta Cir Bras 2016; 31: 417-421

[290] Akbarzadeh S, Bazzi P, Daneshi A, Nabipour I, Pourkhalili K, Mohebbi GH, Sartavi K, Abdi MR, Mirzaei M, Bargahi A. Anti-diabetic effect of Otostegia persica extract on diabetic rats. J Med Plants Res 2012; 6: 3176-3180

[291] Ebrahimpoor-Mashhadi MR, Khaksar Z, Noorafshan A, Mogheisi B. Stereological study of the effects of orally administrated Otostegia persica extract on pancreatic beta cells in male diabetic rats. Comp Clin Pathol 2014; 23: 761-767

[292] Rezaian M, Shomali T, Dilmaghanian A, Rassouli A. Ethanolic extract of Otostegia persica ameliorates bone loss in diabetic rats irrespective to its glucose lowering effect. Comp Clin Pathol 2014; 23: 1147-1151

[293] Shewamene Z, Abdelwuhab M, Birhanu Z. Methanolic leaf extract of Otostegia integrifolia Benth reduces blood glucose levels in diabetic, glucose loaded and normal rodents. BMC Complement Altern Med 2015; 15: 19

[294] Bhatt UP, Sati SC, Chandra S, Kumar S, Anthwal A, Singh R, Singh D, Kumar N, Kumar A, Bahuguna RP. Evaluation of in vivo and in vitro anti-diabetic activity of Roylea cinerea. Int J Pharm Sci Rev Res 2015; 32: 33

[295] Suthar S, Patel R. The antihyperlipidaemic activity of aqueous Leucas urticifolia extract in Triton WR-1339 induced hyperlipidaemic rats. Int J Pharm Technol 2012; 4: 4314-4324

[296] Qazan WSh. Effects of short and long term treatment of Ballota undulata on female albino rats fertility and pregnancy. Pak J Biol Sci 2008; 11: 638-642
[297] Ganjali A, Sotoudeh A, Jahanshahi A, Takhtfooladi MA, Bazzazan A, Roodbari N, Harati MP. Otostegia persica extraction on healing process of burn wounds. Acta Cir Bras 2013; 28: 407-411

[298] Takhtfooladi MA, Takhtfooladi HA, Moayer F, Karimi P, Asl HA. Effect of Otostegia persica extraction on renal injury induced by hindlimb ischemia-reperfusion: a rat model. Int J Surg 2015; 13: 124-130

[299] Safaeian L, Ghasemi-Dehkordi N, Javanmard ShH, Namvar H. Antihypertensive and antioxidant effects of a hydroalcoholic extract obtained from aerial parts of Otostegia persica (Burm.) Boiss. Res Pharm Sci 2015; 10: 192-199

[300] Yeshanew S, Mekonnen Y. The effect of Otostegia integrefolia leaf extracts on the packed cell volume, body weight and survival time of Plasmodium berghei infected mice. Int J Trop Med 2013; 8: 129-134

[301] Nateghpour M, Farivar L, Souri E, Hajjaran H, Mohebali M, Haghi AM. The effect of Otostegia persica in combination with chloroquine on chloroquine-sensitive and chloroquine-resistant strains of Plasmodium berghei using in-vivo fixed ratios method. Iranian J Pharm Res 2012; 11 : 583-588

[302] Dua VK, Verma G, Agarwal DD, Kaiser M, Brun R. Antiprotozoal activities of traditional medicinal plants from the Garhwal region of North West Himalaya, India. J Ethnopharmacol 2011; 136: 123-128

[303] Bezenjani SN, Pouraboli I, Afshar RM, Mohammadi G. Hepatoprotective effect of Otostegia persica Boiss. shoot extract on carbon tetrachloride-induced acute liver damage in rats. Iranian J Pharm Res 2012; 11: 1235-1241

[304] Akbartabar Toori M, Joodi B, Mehraban F, Mostafazadeh M, Ghavamizadeh M, Sadeghi H, Jafari M, Sadeghi H, Talebianpoor MS. Hepatoprotective activity of aerial parts of Otostegia persica against carbon tetrachloride-induced liver damage in rats. Avicenna J Phytomed 2015; 5: 238-246

[305] Upadhyay G, Malik J, Joshi R, Lakshmayya L, Singh UK. Hepatoprotective potential of lyophilized hydro-alcoholic extract of Roylea elegans Wall. against $\mathrm{CCl}_{4}$ and PCM induced hepatotoxicity in Wistar rats. Ann Pharmacol Pharmaceut 2017; 2: 1045

[306] Hashemi F, Zarei MA. Tyrosinase inhibitory activity within hexane extract of ten screened plants from Kurdistan Province of Iran. Int J Adv Biol Biomed Res 2014; 2: 2795-2799

[307] de Sales PM, de Souza PM, Simeoni LA, Magalhaes PDO, Silveira D. $\alpha$-Amylase inhibitors: a review of raw material and isolated compounds from plant source. J Pharm Pharm Sci 2012; 15: 141-183 